\title{
PHASE-LOCKED MOIRE' FRINGE ANALYSIS FOR AUTOMATED CONTOURING OF DIFFUSE SURFACES
}

\author{
by
}

\author{
Bruce Edward Truax \\ Submitted in Partial Fulfillment \\ of the \\ Requirements for the Degree \\ MASTER OF SCIENCE
}

\author{
Supervised by Professor Duncan T. Moore \\ The Institute of Optics \\ The University of Rochester \\ Rochester, New York
}




\section{VITAE}

The author was born in Troy, New York on September 14, 1955. He attended primary and secondary school in Burnt Hills, New York. He received his B.S. in Optics from the University of Rochester in 1976. He was a graduate student at the University of Rochester from January to December 1977. His graduate studies were supported by Western Electric Company in Princeton, New Jersey where he is currently employed. 


\section{ACKNOWLEDGMENT}

At the University of Rochester I would like to thank my advisor and softball teammate Dr. Duncan Moore whose guidance made this work possible. I also thank my fellow students in the Gradient Index Laboratory who helped me during the course of the year. In particular, I thank Glen Johnson for his help in the modifications and redesign of the PLIM Electronics and Danette Ryan for her assistance with the software.

In addition I would like to thank Evie Snyder for putting up with long equations and $z^{\prime} s$ that look like 2's while typing this thesis.

Finally, I thank my father for 22 years of support and encouragement without which this would not have been possible. 


\begin{abstract}
Phase-Locked moire' systems have the advantage of rapid data acquisition and accuracy of better than one twentieth of the fringe spacing. In conventional moiré systems data acquisition is performed by making a photographic plate and taking measurements from the plate either visually or with a microdensitometer. This is slow and the accuracy is about one-quarter of the fringe spacing. The phase-locked system is more accurate and permits a more favorable trade off between accuracy and working depth. The equations governing moire' contouring are examined and compared with the results from interferometric methods. It is found that surface contouring with straight line gratings is equivalent to interferometric contouring in a Twyman-Green type system when certain relations are noted. This equivalence allows many of the current A.C. interferometric techniques to be applied to moire' contouring. The design and operation of a phase-locked Moire system based on phase-locked interferometry is presented.
\end{abstract}




\section{TABLE OF CONTENTS}

VITAE

Page

ACKNOWLEDGMENTS

ii

ABSTRACT

iii

LIST OF FIGURES

CHAPTER

I. INTRODUCTION 1

1.1 Introduction 1

1.2 Description of Moiré Contouring Systems 8

$\begin{array}{ll}1.3 \text { State of the Art } & 10\end{array}$

II. THEORY 13

2.1 Contour Formation 13

2.2 Diffraction Effects 25

2.3 Errors 28

2.4 Phase-Locked Moiré 31

III. EXPERIMENTAL WORK 49

3.1 Design Concepts 49

3.2 Optical System 54

3.2.1 The Fabry-Perot 57

3.2.2 The Scanning System 58

3.2.3 Imaging System 59 
3.3 Electronics and Signal Processing 62

3.3.1 Analog Processor 62

3.3.2 Fringe Counter 66

3.3.3 Image Scanning Electronics 67

3.3.4 Computer Interface 68

3.3.5 Analog to Digital Converter 70

3.4 Data Processing 73

IV. RESULTS 75

4.1 Optical System and Electronics 76

4.2 Measurements and Analysis 79

V. SUMMARY 94

5.1 Conclusion 95

$\begin{array}{ll}\text { APPENDICES } & 100\end{array}$

REFERENCES 153

$\begin{array}{ll}\text { BIBLIOGRAPHY } & 155\end{array}$ 


\section{LIST OF FIGURES}

FIGURE

Page

Arrangement for Generating Moiré Contours

Arrangement for Generating Moiré Contours with Source and Observer at Infinity

2-5 Fundamental and First Harmonic Frequency Components of an Oscillating Moiré Fringe Pattern

2-6 Comparison of Moire' and Interferometry 44

2-7 Amplitude of Fundamental Frequency Component versus Fringe Number

2-8 Concepts of Phase-Locked Moire' Fringe Analysis 46

3-1 Schematic of Optical System 55

3-2 Photograph of Optical System 56

3-3 Layout of Scanning System 60

3-4 Block Diagram of Electronics 63

3-5 Photograph of Electronics 72

4-1 Contour of Spherical Surface without 81

4-2 Contour of Spherical Surface with

Error Voltage Correction

4-3 Example of Contour used to Check Retrace Accuracy

4-4 Example of Resolution in the 1-10 Micron Range 
4-5 Contour of Calibration Sample 88

4-6 Contour of Quarter 90

4-7 Contour of Gear Cutter 91

4-8 Example of Contour Made with a 93 Chrome on Glass Grating 
CHAPTER 1

\subsection{INTRODUCTION}

Non-contact, non-destructive measurement of diffuse surfaces by automated techniques is an important area of metrology. In optics, for example, there is great demand for a system that is capable of measuring profiles of ground glass surfaces to a vertical accuracy of one micron. Such a system bridges the gap between templates and interferometers and also allows a more accurate surface to be generated on the lens in the grinding stage. An automated measurement system is important in the machine tool industry. A clay model or prototype part needs to be rapidly contoured, and this information is stored in a computer to control the machining process so that a duplicate of the sample piece is fabricated. Upon completion of machining, the new part is contoured and compared with the original for quality control purposes.

A great variety of techniques now exist for contouring surfaces. Basically they fall into two categories, contact and non-contact. The contact techniques include methods such as templates and mechanical probes such as micrometers, dial gauges and spherometers. 
The majority of non-contact techniques are optical systems and this again can be divided into two categories, imaging systems and interference systems. Two examples of imaging systems now in use are autofocusing and stereo photogrammetry. Autofocusing is a method by which the elevation of a point on a sample is determined by automatically focusing an imaging system on the point of interest. The elevation is then a function of the position of the focusing mechanism. Stereo photogrammetry is employed by map makers to determine the topography of the earth's surface although it is also applicable to contouring on a smaller scale. The first step in stereo photogrammetry is to take two photographs of the object from slightly different positions in a plane parallel to the surface. From these two photographs it is then possible to determine the elevation of any point present in both photographs by the difference in parallax of the two images .

The most widely used interference method for contouring surfaces is the interferometer. It is capable of contouring specular surfaces to an accuracy of one twentieth of the wavelength of light. Another useful interferometric technique is holography which is a method by which a three dimensional image of the object is recorded. This three dimensional image then is interpreted in a variety of different ways to yield contour information. The hologram 
can also be used to compare the original object with other similar ones in a method similar to interferometry. The hologram is used to form a reference wave which can interfere with light scattered from the object. The result of this procedure is an image with contour fringes representing the differences in the original object and the new sample.

Another interference technique is the method of "projected fringes"10 developed by Haines and Hildebrand. 16 This system involves generating planes of light and dark in space by interfering two beams of mutually coherent light at slight angles to each other. If an object is placed in this region of interference the fringes are projected onto the surface forming a contour map.

One of the more useful forms of surface measurement is contouring a surface with lines of equal spacing. The ease of such measurements for diffuse surfaces has been significantly increased by the development of moire' topographic techniques. The term moire' was derived from a fabric of the same name which produces light and dark fringes when two pieces are held in contact.

Moiré fringes produced by two diffraction gratings in contact with one another were first noted and documented by Lord Rayleigh in 1874. In his article "On the Manufacture and Theory of Diffraction Gratings" 1 Lord Rayleigh writes of the rulings on a grating with 3000 
lines per inch:

"The lines themselves are of course too close to be seen without a microscope; but their presence may be detected, and even the interval between them measured, without optical aid, by a method not depending on the production of spectra or requiring a knowledge of the wavelength of light. If two photographic copies (replicated gratings) containing the same number of lines to the inch be placed in contact, film to film, in such a manner that the lines are nearly parallel in the two gratings, a system of parallel bars develops itself, whose direction bisects the external angle between the directions of the original lines, and whose distance increases as the angle of inclination diminishes. . The interval between the bars is evidently half the long diagonal of the rhombus formed by two pairs of consecutive lines, and is expressed by $a \cos \frac{1}{2} \theta \div \sin \theta$, or approximately $a \div \theta$ where $a$ is the interval between the primary lines, and $\theta$ the mutual inclination of the two sets.

When parallelism is very closely approached the bars become irregular, in consequence of imperfection of the ruling. This phenomenon might perhaps be made useful as a test."

Lord Rayleigh was correct in assuming that those fringes he saw would be useful as a test for the quality of diffraction gratings but it wasn't until 1923 that this theory was developed and published in an article by J.A. Anderson entitled "The Manufacture and Testing of Diffraction Gratings". 2 The test was used to check the quality of the gratings produced by a ruling engine. His test was accomplished by ruling two gratings onto the same blank, the second inclined at an angle of about one minute to the first. Anderson developed the mathematical relationships describing the characteristics of the moire' pattern produced by the types of errors most commonly produced by ruling engines such as periodic error, linear error 
and accidental errors.

In the late 1930's the use of moire' fringes was extended into the field of strain analysis. For this application a grating (usually a piece of printers process screen with about 200 lines/inch) was printed directly on the sample to be studied. This grating was then viewed through another piece of the screen or "photo-grid", as it was sometimes called. As stress was applied to the sample moire' patterns appeared due to the deformation of the printed grid. The moire' patterns thus formed were an indication of the deformation of the material. This test had the disadvantage that it was destructive because the grating was etched or printed directly on the sample to be studied. ${ }^{3}$

The first use of moire' as a test for replica gratings (not master gratings as Anderson described) was described in an article by Merton ${ }^{4}$ in 1950. Merton had developed a new method for the production of gratings which eliminated periodic error from the rulings. He used moire' testing methods, which are sensitive to periodic error, to test his new gratings.

It wasn't until 1956 that a detailed treatment of the Theory of Moire' fringes formed by crossed gratings was published. In a book by Guild entitled The Interference Systems of Crossed Diffraction Gratings, Theory of Moiré Fringes the author considered the case of two gratings in 
series treating, in detail, both coherent and incoherent illumination.

Surface contours have been generated by moire' methods. Theocaris ${ }^{15}$ describes a system with only one grating which is shadowed onto an object and this shadow is then viewed through the grating. A very simple derivation of the location of the moire' fringes is given. Since Theocaris was primarily interested in studying the effects of stress on materials, he did not develop the possibility of surface contouring with his apparatus.

In 1967 Daniel Post expanded on Guild's work in multiplication of moiré fringes. ${ }^{13,14}$ Post studied a fringe multiplication technique which, by using coherent light to illuminate two gratings in series, increased the frequency of the moire' fringes by an order of magnitude causing an equivalent increase in the sensitivity of the moire' test. These papers also discussed fringe multiplication when the frequency of the two gratings differ by an integral multiple. Note that Post's work only deals with two transparent gratings in series and is not applicable to surface contouring or strain analysis.

The next major advance in moire' metrology came in 1970 when, within two months of each other two papers on Surface Contouring by Moiré Patterns ${ }^{5,6}$ were published. Both of these papers develop the theory of moire' surface 
contouring showing how, under certain conditions moire' patterns can be produced which exactly represent height contours on a surface. 


\subsection{Description of Moire' Contouring Systems}

The procedure for creating moire' contour fringes on a surface is relatively simple. The object to be contoured is placed behind a grating consisting of equally spaced parallel lines. (Figure 2-1) This system is illuminated with a beam of parallel light through the grating. On the surface of the object a shadow of the grating is formed and the lines of the shadow curve conforming to the shape of the object. If the object is viewed through the grating from a distance which is large compared to the grating to object distance, a series of equally spaced contours are observed. These contours are due to the moire' pattern formed by the multiplication of the transmission function of the grating and the intensity function of the deformed shadow of the grating which is present on the object.

The spacing of the contours can be easily varied by changing either the grating spacing or the angle between the illuminating beam and the viewer. The contour separation is directly proportional to the grating spacing and inversely proportional to the sum of the tangents of the angles between the viewer and illumination with respect to the normal at the grating surface.

There are a few disadvantages to this form of contouring. The most important is the surface to be measured must be a diffuse reflector to some extent. For 
example, a specular surface such as a mirror cannot be contoured but a coin such as a nickel or a quarter which has slight diffuse reflectance can be contoured. Also translucent objects tend to have very low contrast fringes because the internal scattering washes out the grating's shadow. Another problem which limits the usefulness of this technique is the low contrast of the fringes at large grating-object spacings due to diffraction by the lines of the grating. Objects which have very steep slopes also cause problems. As the slope increases, the horizontal spacing of the contours decreases until it is near the same size as the grating spacing. At this point the two patterns can no longer be separated. A final problem is familiar to users of interferometers. If there are less than two fringes present in the aperture then the fringe pattern is difficult to interpret. 


\subsection{State of the Art}

Since 1970 the majority of work on moiré contouring has been with static systems where all data acquisition is done by photography and data reduction is slow and tedious. Variations of this basic technique include projecting the fringes onto the object and then photographing the sample with the grating lines on it. The negative is then viewed at a later time through a selection of different gratings. This modification is useful for contouring large objects without having to manufacture gratings of comparable size. ${ }^{7}$

Another variation involves projecting two identical gratings onto an object from two separate angles. This forms a contour on the surface by the addition of intensities of the two patterns. This contrasts the previous technique in which intensities are multiplied. The advantage here is that very large objects can be contoured if each of the grating patterns are produced by the interference of two collimated light beams. ${ }^{8}$

The most recent advance in moiré Topography is a system for automatic data acquisition developed by Idesawa, Yatagui and Suma ${ }^{9}$ in Japan. This system employs a projected grating which is then viewed with a flying-spot scanner T.V. camera. The second grating is produced by the raster lines of the scan. The information obtained from the camera is then processed by a computer to give a 
contour map of the surface. ${ }^{9}$

The resolution of contour maps generated by any of these oire' techniques is limited by certain inherent characteristics of the oire'patterns. The total resolution of any contouring system is a combination of two factors, horizontal resolution and vertical resolution. The vertical resolution is determined by the accuracy with which the phase of the fringe pattern can be determined. For the current state of the art this is about one-quarter to one-tenth of the fringe spacing. The improvement of this resolution is one of the major objectives of this study.

At first glance the horizontal resolution would appear to be limited by the f-stop of whatever imaging system is used to observe the oire' pattern but this is not necessarily the case. If the imaging system resolves the lines of the grating, then the fringes observed are those of the grating and not the oire' pattern. The smallest resolvable spot has to be at least twice the spacing of the lines on the grating. This restriction can be eliminated by moving the grating in its plane perpendicular to the grating lines during observation. As will be shown in Chapter 2, the iré fringes are position invariant for grating movement if the grating to object distance does not change. Under these circumstances the horizontal resolution is limited only by the f-stop 
of the viewing system.

In this thesis a system is described which offers increased vertical resolution of oire' contours and automatically contours a surface allowing either a real time output of the surface contour to an analog display device or a digital output to a computer which analyzes the data. 
CHAPTER II

\section{THEORY}

\subsection{Contour Formation}

An arrangement for generating standard moire' contours on a surface is pictured schematically in Figure 2-1. The illuminator is a point source located at position $S$ and the observer views the pattern from the point designated 0 . The grating is expressed by a generalized periodic intensity transmittance function of the form:

$$
T(x, y)=\frac{1}{2}+\frac{1}{2} g\left(\frac{2 \pi}{p} x\right),
$$

where $p$ is the period of the grating and $g$ lies between plus and minus one. This implies the rulings on the grating are parallel to the $\mathrm{x}$-axis and lie in the $\mathrm{x}-\mathrm{y}$ plane. The light emitted by the point source at $\mathrm{S}$ casts a shadow of the grating onto the surface of the object. The point on the grating with $\mathrm{x}$ coordinates $\left(\mathrm{x}_{0}, \mathrm{y}_{0}, 0\right)$ is translated to a point $\mathrm{P}$ with coordinates $\left(\mathrm{x}_{1}, \mathrm{y}_{1}, \mathrm{z}_{1}\right)$ on the surface and by similar triangles $\mathrm{x}_{0}$ and $\mathrm{x}_{1}$ are related by: 


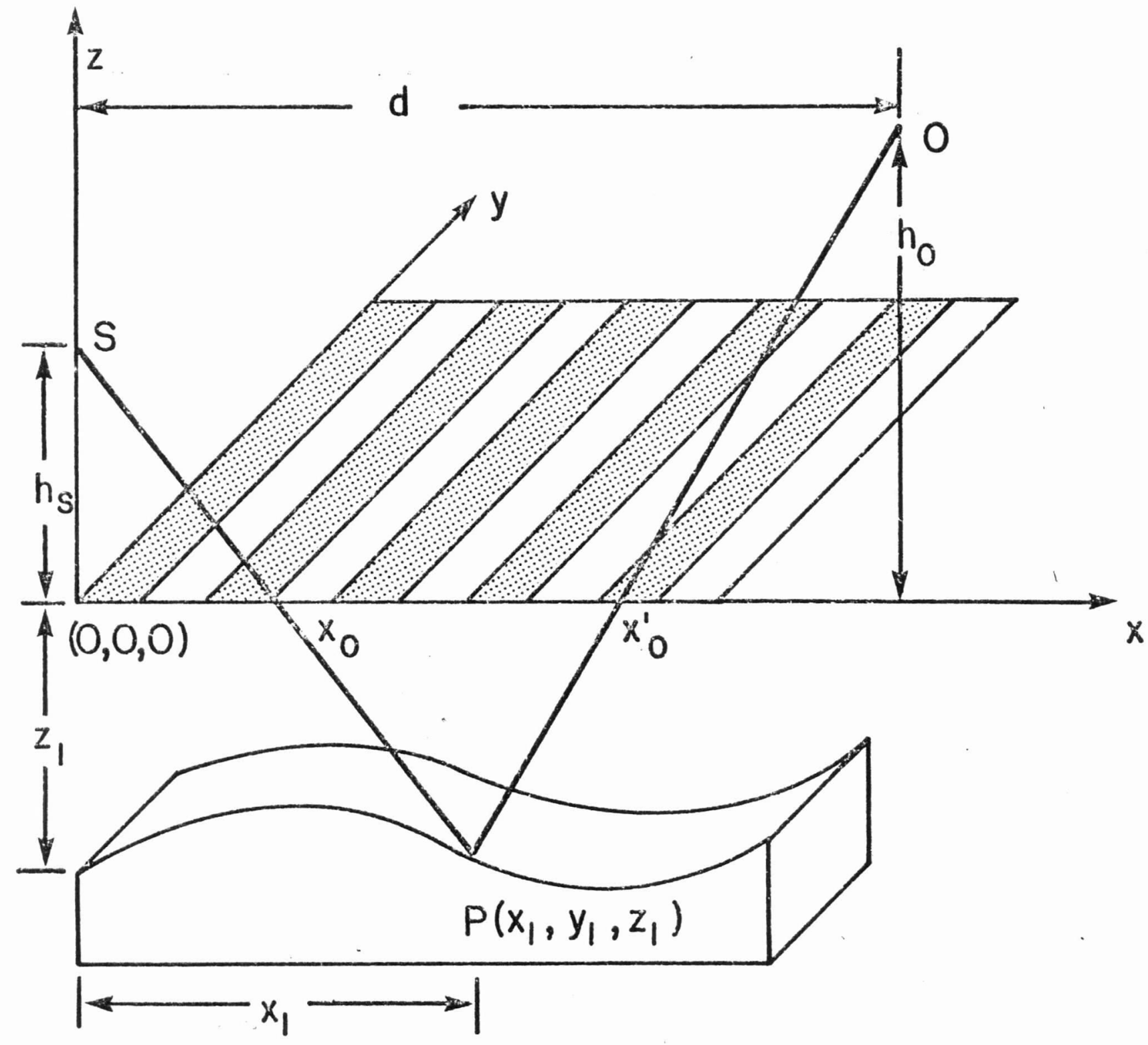

Arrangement for Generating Moiré Contours

Figure 2-1 


$$
\frac{h_{s}}{x_{0}}=\frac{h_{s}+z_{1}}{x_{1}}+x_{0}=\frac{x_{1} h_{s}}{h_{s}+z_{1}} .
$$

If the intensity of the point source is $I_{0}$ and $\Phi\left(\mathrm{x}_{1}, \mathrm{y}_{1}, \mathrm{z}_{1}\right)$ is the angle between the surface normal and the incoming light ray, then the irradiance at any point $P$ on the object is:

$$
I_{1}=I_{0} \cos \Phi\left\{\frac{1}{2}+\frac{1}{2} g\left(\frac{2 \pi}{p} \frac{x_{1} h_{s}}{\left(h_{s}+z_{1}\right.}\right)\right\} .
$$

Light scattered from the point $\mathrm{P}\left(\mathrm{x}_{1}, \mathrm{y}_{1}, \mathrm{z}_{1}\right)$ to the observer passes through the point on the grating with coordinates $\left(x_{0}{ }^{\prime}, y_{0}{ }^{\prime}, 0\right)$. These two points are related by the equation

$$
\frac{h_{0}+z_{1}}{d-x_{1}}=\frac{h_{0}}{d-x_{0}} \rightarrow x_{0}^{\prime}=\frac{h_{0} x_{1}+z_{1} d}{h_{0}+z_{1}} .
$$

The irradiance at point 0 is given by:

$$
I_{2}=I_{1}\left\{\frac{1}{2}+\frac{1}{2} g\left(\frac{2 \pi\left(h_{0} x_{1}+z_{1} d\right)}{p\left(h_{0}+z_{1}\right)}\right)\right\}
$$

Substituting Eq. (3) into Eq. (5) the resulting irradiance pattern is: 


$$
\begin{aligned}
& I_{2}=\frac{I_{0} \cos \Phi\left(x_{1}, y_{1}, z_{1}\right)}{4}\left\{1+g\left(\frac{2 \pi x_{1} h_{s}}{p\left(h_{s}+z_{1}\right)}\right)\right. \\
& \left.\quad+g\left(\frac{2 \pi}{p} \frac{x_{1} h_{0}+z_{1} d}{h_{0}+z_{1}}\right)+g\left(\frac{2 \pi}{p} \frac{x_{1} h_{s}}{h_{s}+z_{1}}\right) \cdot g\left(\frac{2 \pi}{p} \frac{x_{1} h_{0}+z_{1} d}{h_{0}+z_{1}}\right)\right\} .
\end{aligned}
$$

Since $g$ is perodic and finite it can be expressed by the Fourier Series:

$$
g\left(\frac{2 \pi}{p} x\right)=\sum_{n=1}^{\infty}\left(a_{n} \sin \left(\frac{2 \pi n x}{p}\right)+b_{n} \cos \left(\frac{2 \pi n x}{p}\right)\right) .
$$

Substituting (6) into (7):

$$
\begin{aligned}
& I_{2}=\frac{I_{0} \cos \Phi}{4}\left\{1+\sum_{n=1}^{\infty}\left[a_{n}\left(\sin \frac{2 \pi n x_{1} h_{s}}{p\left(h_{s}+z_{1}\right)}+\sin \frac{2 \pi n}{p}\left(\frac{x_{1} h_{0}+z_{1} d}{\left(h_{0}+z_{1}\right)}\right)\right)\right.\right. \\
& +b_{n}\left(\cos \frac{2 \pi n x_{1} h_{s}}{p\left(h_{s}+z_{1}\right)}+\cos \frac{2 \pi n}{p}\left(\frac{h_{0} x_{1}+z_{1} d}{h_{0}+z_{1}}\right)\right] \\
& +\sum_{n=1}^{\infty} \sum_{m=1}^{\infty}\left[\left(a_{n} \sin \frac{2 \pi n x_{1} h_{s}}{p\left(h_{s}+z_{1}\right)}+b_{n} \cos \frac{2 \pi n x_{1} h_{s}}{p\left(h_{s}+z_{1}\right)}\right)\right. \\
& \cdot\left(a_{m} \sin \frac{2 \pi m}{p}\left(\frac{h_{0} x_{1}+z_{1} d}{h_{0}+z_{1}}+b_{m} \cos \frac{2 \pi m}{p}\left(\frac{h_{0} x_{1}+z_{1} d}{h_{0}+z_{1}}\right)\right]\right\}
\end{aligned}
$$


Working only with the double summation term:

$$
\begin{aligned}
& \cdots+\sum_{m=1}^{\infty} \sum_{n=1}^{\infty}\left\{a_{n} a_{m}\left[\sin \frac{2 \pi n h_{s} x_{1}}{p\left(h_{s}+z_{1}\right)}\right] \cdot\left[\sin \frac{2 \pi m}{p}\left(\frac{h_{0} x_{1}+z_{1} d}{h_{0}+z_{1}}\right)\right]+\right. \\
& b_{n} b_{m}\left[\cos \frac{2 \pi n h_{s} x_{1}}{p\left(h_{s}+z_{1}\right)}\right] \cdot\left[\cos \frac{2 \pi m}{p}\left(\frac{h_{o} x_{1}+z_{1} d}{h_{o}+z_{1}}\right)\right]+ \\
& a_{n} b_{m}\left[\sin \frac{2 \pi n h_{s} x_{1}}{p\left(h_{s}+z_{1}\right)}\right] \cdot\left[\cos \frac{2 \pi m}{p}\left(\frac{h_{0} x_{1}+z_{1} d}{h_{0}+z_{1}}\right)\right]+ \\
& \left.b_{n} a_{m}\left[\cos \frac{2 \pi n h_{s} x_{1}}{p\left(h_{s}+z_{1}\right)}\right] \cdot\left[\sin \frac{2 \pi m}{p}\left(\frac{h_{o} x_{1}+z_{1} d}{h_{0}+z_{1}}\right)\right]\right\}
\end{aligned}
$$

Using the trigonometric identities for the sums and differences of two angles and splitting up the double sumation Eq. (8) can be expressed as: 


$$
\begin{aligned}
& I_{2}=\frac{I_{0} \cos \phi}{4}\left\{1+\sum_{n=1}^{\infty}\left[a_{n}\left(\sin \frac{2 \pi n h_{s} x_{1}}{p\left(h_{s}+z_{1}\right)}+\sin \frac{2 \pi n}{p} \frac{h_{0} x_{1}+z_{1} d}{h_{0}+z_{1}}\right)\right.\right. \\
& \left.+b_{n}\left(\cos \frac{2 \pi n h_{s} x_{1}}{p\left(h_{s}+z_{1}\right)}+\cos \frac{2 \pi n}{p} \frac{h_{0} x_{1}+z_{1} d}{h_{0}+z_{1}}\right)\right] \\
& +\quad \sum_{n=1}^{\infty}\left\{\frac{a_{n}^{2}+b_{n}^{2}}{2} \cos \left[\frac{2 \pi n}{p}\left(\frac{\left(h_{s}^{-} h_{0}\right) z_{1} x_{1}}{\left(h_{s}+z_{1}\right)\left(h_{0}+z_{1}\right)}-\frac{z_{1} d}{h_{0}+z_{1}}\right)\right]\right. \\
& +a_{n} b_{n} \sin \left[\frac{2 \pi n}{p}\left(\frac{\left(h_{s}+h_{0}\right) z_{1} x_{1}+2 h_{0} h_{s} x_{1}}{\left(h_{s}+z_{1}\right)\left(h_{0}+z_{1}\right)}+\frac{z_{1} d}{h_{0}+z_{1}}\right)\right] \\
& \left.+\frac{b_{n}^{2}-a_{n}^{2}}{2} \cos \left[\frac{2 \pi n}{p}\left(\frac{\left(h_{s}+h_{0}\right) z_{1} x_{1}+2 h_{0} h_{s} x_{1}}{\left(h_{s}+z_{1}\right)\left(h_{0}+z_{1}\right)}+\frac{z_{1} d}{h_{0}+z_{1}}\right)\right]\right\} \\
& +\sum_{n=1}^{\infty} \sum_{\substack{n \neq m \\
n \neq 1}}^{\infty}\left\{\frac{a_{m} a_{n}+b_{n} b_{m}}{2} \cos \left[\frac{2 \pi}{p}\left(\frac{\left(n h_{s}-m h_{0}\right) z_{1} x_{1}+2 h_{o} h_{s}(m-n) x_{1}}{\left(h_{s}+z_{1}\right)\left(h_{o}+z_{1}\right)}-\frac{m z_{1} d}{h_{0}+z_{1}}\right)\right]\right. \\
& +a_{m} b_{n} \sin \left[\frac{2 \pi}{p}\left(\frac{\left(n h_{s}+m h_{0}\right) z_{1} x_{1}+2 h_{0} h_{s}}{(m+n) x_{1}}+\frac{m z_{1} d}{\left.h_{s}+z_{1}\right)\left(h_{0}+z_{1}\right.}\right)\right] \\
& \left.\left.+\frac{b_{m} b_{n}-a_{m} a_{n}}{2} \cos \left[\frac{2 \pi}{p}\left(\frac{\left(n h_{s}+m h_{0}\right) z_{1} x_{1}+2 h_{0} h_{s}(m+n) x_{1}}{\left(h_{s}+z_{1}\right)\left(h_{0}+z_{1}\right)}+\frac{m z_{1} d}{h_{0}+z_{1}}\right)\right]\right\}\right\} .
\end{aligned}
$$


Notice that there is no term in Eq. (10) which is dependent solely on $z_{1}$ and therefore there is no simple way to interpret the moiré pattern formed. If the source and observation point are at the same height above the grating, then the first term in the second summation in Eq. (10) loses its $x$ dependence and the expression for $I_{2}$ becomes:

$$
\begin{aligned}
& I_{2}=\frac{I_{0} \cos \Phi}{4}\left\{1+g\left(\frac{2 \pi h x_{1}}{p\left(h+z_{1}\right)}\right)+g\left(\frac{2 \pi}{p} \frac{\left(d z_{1}+h x_{1}\right)}{\left(h+z_{1}\right)}\right)+\right. \\
& \sum_{n=1}^{\infty}\left\{\frac { a _ { n } { } ^ { 2 } + b _ { n } { } ^ { 2 } } { 2 } \operatorname { c o s } \left[\frac{2 \pi n}{p} \frac{z_{1} d}{\left(h+z_{1}\right]}+\right.\right. \\
& \left.+b_{n} \sin \frac{2 \pi n}{p}\left(\frac{d z_{1}+2 h x_{1}}{h+z_{1}}\right)+\frac{b_{n}{ }^{2}-a_{n}{ }^{2}}{2} \cos \frac{2 \pi n}{p}\left(\frac{d z_{1}+2 h x_{1}}{h+z_{1}}\right)\right\} \\
& +\sum_{n=1}^{\infty} \sum_{n \neq m}^{\infty}\left\{\left(\frac{a_{m} a_{n}+b_{n} b_{m}}{2}\right) \cos \frac{2 \pi}{p}\left(\frac{(m-n) h x_{1}-n d z_{1}}{h+z_{1}}\right)\right. \\
& +\frac{b_{m} b_{n}-a_{m} a_{n}}{2} \cos \frac{2 \pi}{p}\left(\frac{(m+n) h x_{1}+n d z_{1}}{h+z_{1}}\right) \\
& +a_{m} b_{n} \sin \frac{2 \pi}{p}\left(\frac{(m+n) h x_{1}+n d z_{1}}{h+z_{1}}\right)
\end{aligned}
$$


If the slope of the surface is less than $\mathrm{h} / \mathrm{d}$, then all of the terms with an $x_{1}$ dependence have higher frequency variations than the term which is only dependent on $z_{1}$. They can be removed either by spatial filtering (coherent illumination only) or through the use of an imaging system which does not resolve the higher frequencies. Once this is done Eq. (11) is reduced to:

$$
I_{2}=\frac{I_{0} \cos \Phi}{4}\left\{1+\sum_{n=1}^{\infty}\left(\frac{a_{n}{ }^{2}+b_{n}{ }^{2}}{2} \cos \frac{2 \pi n d z_{1}}{p\left(h+z_{1}\right)}\right)\right\} .
$$

The contouring interval is found by setting the argument of the cosine at the fundamental frequency $(n=1)$ equal to $2 \pi N$ where $N=0,1,2,3 \ldots$

$$
\frac{2 \pi d\left(z_{1}+\Delta z\right)}{p\left(h+z_{1}+\Delta z\right)}-\frac{2 \pi d\left(z_{1}\right)}{p\left(h+z_{1}\right)}=2 \pi(N+1)-2 \pi N,
$$

this simplifies:

$$
\Delta z=\frac{-p z_{1}^{2}-2 p z_{1} h-p h^{2}}{p h+p z_{1}-d h}
$$


In this case the contour interval is a function of $z_{1}$ but in most applications $h>>z_{1}$ and the $z_{1}$ dependence disappears giving:

$$
\Delta z=\frac{-p h}{p-d}
$$

Also since $p$ is the period of the grating, usually $\mathrm{d}>>\mathrm{p}$ giving the final expression for $\Delta z$ as:

$$
\Delta z=\frac{t p h}{\mathrm{~d}}
$$

There are basically two types of gratings used to generate moire' patterns for contouring surfaces - the types with sinusoidal density variations such that

$$
T(x)=\frac{1}{2}+\frac{1}{2} \cos \left(\frac{2 \pi x}{p}\right)
$$

and Ronchi Rulings which have a square wave transmittance function equal to:

$$
T(x)=\frac{1}{2}+\frac{1}{\pi} \sum_{n=1}^{\infty} \frac{1}{n} \sin \frac{2 \pi n x_{1}}{p}, .
$$

Substitution of these two grating types give the following two moire' patterns, respectively: 


$$
\begin{gathered}
I_{2_{\text {sine }}}=\frac{I_{0} \cos \Phi}{4}\left(1+\frac{1}{2} \cos \frac{2 \pi z_{1} \mathrm{~d}}{\mathrm{p}\left(\mathrm{h}+\mathrm{z}_{1}\right)}\right) \\
I_{2_{\text {Ronchi }}}=\frac{\mathrm{I}_{\mathrm{O}} \cos \Phi}{4}\left(1+\frac{2}{\pi^{2}} \sum_{\mathrm{n}=1}^{\infty} \frac{1}{\mathrm{n}^{2}} \cos \frac{2 \pi \mathrm{ndz} z_{1}}{\mathrm{p}\left(\mathrm{h}+\mathrm{z}_{1}\right)}\right) .
\end{gathered}
$$

One difference between the two gratings is immediate1y apparent from Eqs. (19) and (20). The maximum contrast for the sinusoidal grating is $\frac{1}{2}$ while the contrast obtained from the Ronchi ruling can be as high as unity. The actual experimental results yield much lower contrast than these ideal numbers but since the Ronchi ruling has inherently higher contrast it is much more desirable for contouring applications.

A simplification which is useful experimentally is the case where the height of the source and object each go to infinity. This corresponds to a collimated illuminating beam incident on the grating at an angle $\alpha$ to the normal and a very large viewing distance. The grating then subtends a very small angle and is observed at an angle $\beta$ (see Figure 2-2).

The contouring function for this case becomes: 


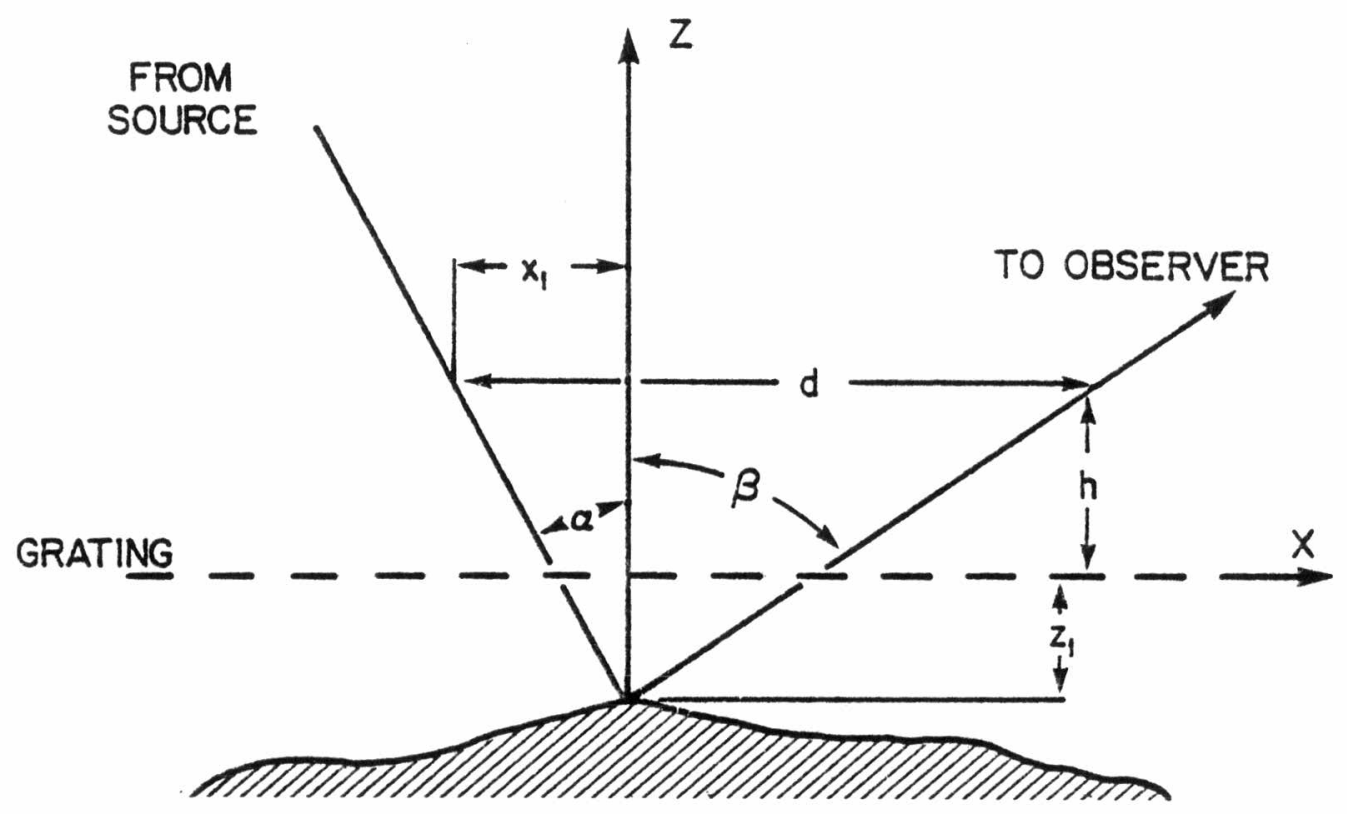

\section{OBJECT}

Arrangement for Generating Moire' Contours with Source and Observer at Infinity

Figure 2-2 
$I_{2}=\frac{I_{0} \cos \Phi}{4}\left[1+\sum_{n=1}^{\infty}\left(\frac{a_{n}{ }^{2}+b_{n}{ }^{2}}{2}\right) \cos \frac{2 \pi n(\tan \alpha+\tan \beta) z_{1}}{p}\right]$

The substitution of $\tan \alpha+\tan \beta$ for $\frac{\mathrm{d}}{\mathrm{h}+\mathrm{z}_{1}}$ is seen if $d-x_{1}+x_{1}$ is substituted for $d$ :

$$
\frac{\mathrm{d}-\mathrm{x}_{1}}{\mathrm{~h}+\mathrm{z}_{1}}+\frac{\mathrm{x}_{1}}{\mathrm{~h}+\mathrm{z}_{1}}=\tan \beta+\tan \alpha
$$

The contouring interval for this case is not dependent on the magnitude of $z$ and is given by the expression

$$
\Delta z=\frac{p}{\tan \alpha+\tan \beta}
$$

Because of the simplified nature of these expressions in comparison to those derived for arbitrary positioning of the source and observer this is the situation considered exclusively in this work. 


\subsection{Diffraction Effects}

The theoretical contrast of the moiré pattern is never observed in practice. The degradation in contrast is due to a loss in sharpness of the shadow pattern caused by diffraction. Erf ${ }^{10}$ treats this problem as one of self-imaging (the phenomena whereby diffraction causes a periodic object to produce an image of itself) and uses one-quarter of the distance at which self-imaging occurs as the maximum contouring depth. This analysis can be applied to the experimental situation presented in this thesis since coherent illumination of the grating is used.

The Ronchi ruling has the transmission function given in Eq. (18). The transmitted wavefront including diffraction effects at a small distance, $z$, from the grid may be described by

$$
A(x)=\frac{A_{0}}{2}\left(1+\frac{2}{\pi} \sum_{\substack{n=1 \\ n \\ \text { odd }}}^{\infty} \frac{1}{n} \sin \left(\frac{2 \pi n x}{p}+\psi(z)\right)\right),
$$

where

$$
\psi_{\mathrm{n}}=\frac{2 \pi}{\lambda} \frac{\mathrm{z}}{\cos \theta_{\mathrm{n}}}
$$

and

$$
\sin \theta_{\mathrm{n}}=\frac{\mathrm{n}}{\lambda \mathrm{p}}
$$

Eq. (25) is the standard diffraction grating equation. For $\theta_{\mathrm{n}}$ small Eqs. (24) and (25) reduce to 


$$
\theta_{\mathrm{n}}=\frac{\mathrm{n}}{\lambda \mathrm{p}}
$$

and

$$
\psi_{\mathrm{n}}=\frac{2 \pi z}{\lambda\left(1-\lambda^{2} \mathrm{n}^{2} / 2 \mathrm{p}^{2}\right)}
$$

The lowest order self-image occurs when $\psi_{1}-\psi_{0}=\pi$. Substituting this condition into Eq. (24a) the self imaging distance reduces to

$$
z=\frac{p^{2}-\lambda^{2}}{\lambda}
$$

For a grating used in Moire contouring

$$
p>>\lambda
$$

and Eq. (26) becomes

$$
z=\frac{p^{2}}{\lambda}
$$

Taking one quarter of this self-imaging distance

$$
z_{\max } \leq \mathrm{p}^{2} / 4 \lambda
$$

The squared dependence of $z_{\max }$ on the grating period is very significant. If the accuracy with which the fringe pattern can be interpreted is doubled, then a 
grating of twice the period can be used and the contouring depth will increase by a factor of four. 


\subsection{Errors}

In order to accurately contour a surface by conventional moiré techniques the contouring interval $\Delta z$ must be known to a high degree of precision. In the calculations of $\Delta z$ for the source and observer at infinity there are basically three sources of error:

1) Error in the determination of the angles $\alpha$ and $\beta$.

If $\varepsilon$ is the uncertainty in $\Delta z$ and $\delta \alpha$ and $\delta \beta$ are the uncertainties in $\alpha$ and $\beta$, then by differentiation

$$
\varepsilon_{\alpha}=\left.\right|^{\delta \Delta z}|=| \frac{\mathrm{p}}{\cos ^{2} \alpha(\tan \alpha+\tan \beta)} \mid
$$

and

$$
\begin{aligned}
& \varepsilon_{\beta}=|\delta \Delta z|=\left|\frac{p}{\cos ^{2} \beta(\tan \alpha+\tan \beta)}\right| \\
& \text { combining } \varepsilon_{\alpha} \text { and } \varepsilon_{\beta} \\
& \varepsilon=\varepsilon_{\alpha}+\varepsilon_{\beta}=\left[\Delta z\left(\left|\frac{\delta \alpha}{\cos ^{2} \alpha}\right|+\left|\frac{\delta \beta}{\cos ^{2} \beta}\right|\right)\right] .
\end{aligned}
$$

2) Error in the measurement of the grating spacing. $p$ or variations in this spacing. The error 
introduced by an uncertainty in the grating spacing of $\delta \mathrm{p}$ is easily shown to be:

$$
\varepsilon_{\mathrm{p}}=\left|\frac{\delta \mathrm{p}}{\tan \alpha+\tan \beta}\right|
$$

3. Error in alignment of the rulings on the grating perpendicular to the illuminating beam.

If $\theta$ is the angle of the normal to the grating rulings with the projection of the incoming beam into the plane of the grating (Figure 2-3) then the error $\varepsilon_{\theta} \quad$ is given by

$$
\Delta z=\frac{p}{\cos \theta} \frac{1}{\tan \alpha+\tan \beta} .
$$

where $\frac{p}{\cos \theta}$ is the effective grating spacing

$$
\varepsilon_{\theta}|\delta \Delta z|=\left|\frac{p \sin \theta}{\cos ^{2} \theta} \frac{1}{\tan \alpha+\tan \beta} \delta \theta\right|
$$

Notice that if $\theta$ is 0 and $\delta \theta$ is $\operatorname{small} \varepsilon_{\theta}$ is equal to zero indicating that $\Delta z$ will be very insensitive to small alignment errors. 


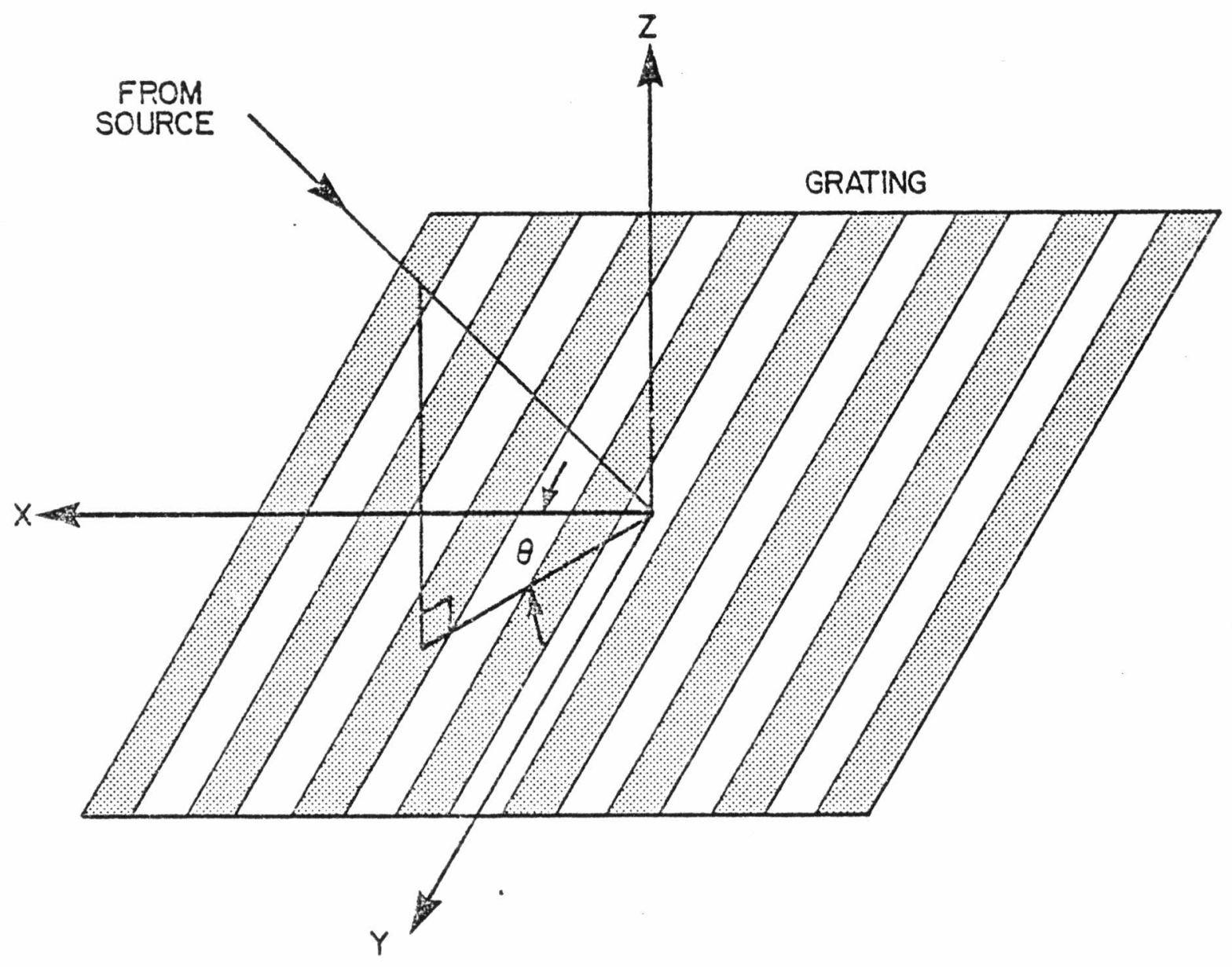

Grating Alignment Error

Figure 2-3 


\subsection{Phase-Locked Moire'}

There are many similarities between moire' surface topography and Interferometric testing. For example, in the case where both the source and observer are at infinity and a sinusoidal grating is employed, Eq. reduces to

$$
I_{2}=\frac{I_{0} \cos \Phi}{4}\left[I+\frac{1}{2} \cos \frac{2 \pi}{p}\left(\frac{\tan \alpha+\tan \beta}{)} z(x, y)\right] .\right.
$$

This equation is similar to that for a Twyman-Green Interferometer with the object as the mirror in one arm (see Fig. $2-4)$.

$$
I_{1}=k\left[1+\cos \frac{4 \pi}{\lambda} z(x, y)\right] \quad .
$$

The only differences between these equations is the lower contrast in the moire' system and the interchange of the half-wavelength $\frac{\lambda}{2}$ and the grating constant $\frac{p}{\tan \alpha+\tan \beta}$. Both of these methods produce a contour map of the surface.

Because of these similarities the methods of data acquisition and reduction used in interferometry can be applied to moire' systems.

The most common acquisition-reduction system now in use is to make a photographic plate of the fringe pattern and then interpret the fringe pattern with a microdensitometer. This method has three very serious drawbacks. The 


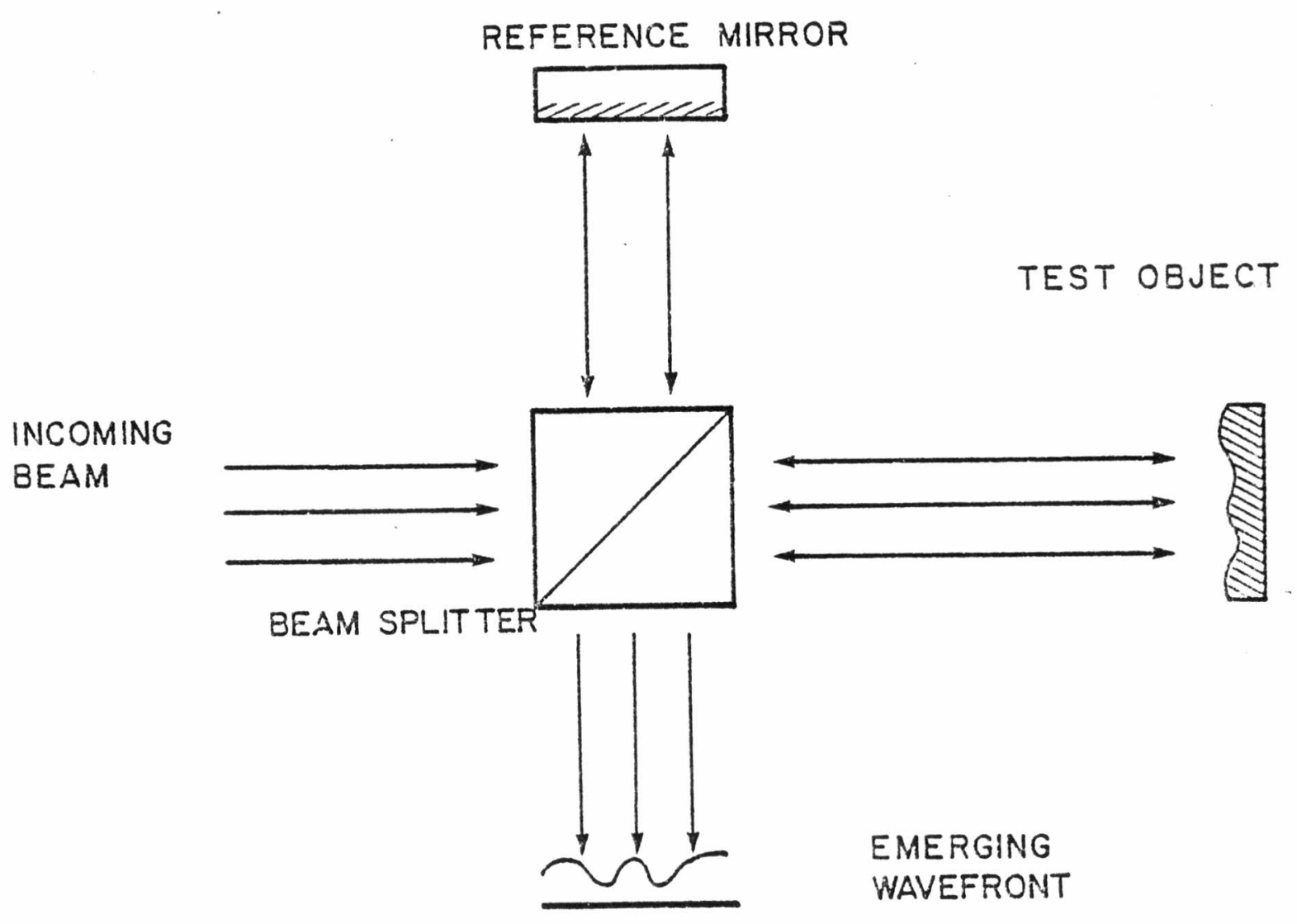

TWYMAN - GREEN INTERFEROMETER

Twyman Green Interferometer

Figure 2-4 
first and most important is the time required to completely contour a surface which can easily run into hours. The second is the accuracy with which the fringe pattern can be interpreted which is usually limited to $1 / 10$ th of a fringe. The third disadvantage is that the slope of the surface between two adjacent fringes cannot be uniquely determined. If the slope of the surface is not known prior to the measurement, then to determine its sign a second plate must be made after the object to grating distance has been shifted by a fraction of the contouring interval. Of course there are many times when a qualitative judgement suffices and a quick glance at the plate or the projected fringe pattern yields the needed information. If quantitative measurements are only rarely needed, then the expense of the microdensitometer may be eliminated and the few plates which need accurate measurements can be evaluated elsewhere.

To speed up this process, photographic plates must be replaced with electronic recording techniques and a computer to analyze the fringe pattern. One method for doing this is to image the fringe pattern onto a vidicon tube which is interfaced to a computer where the pattern is analyzed. This is much quicker than the previous method and the cost of the electronics-computer package is competitive with the cost of a microdensitometer. The accuracy of this system is also limited to about $1 / 10$ th 
of a fringe.

The next approach is a digital interferometer which aims to improve the accuracy of the previous technique by examining the fringe pattern a number of times. For each measurement the reference mirror position is shifted by a fraction of a wavelength. In this configuation the fringe pattern is imaged on a vidicon tube and the image is evaluated by the computer after each shift.

The relative phase between adjacent detectors can be calculated if many images are sampled. From the relative phases a contour map of the surface is generated. This method has several advantages, it is more accurate than any previous method and it is able to distinguish hills from valleys. Also by using a computer for data reduction, it is simple to add or subtract wavefronts for null tests or system tests.

An accurate interferometric technique in use at this time is a system developed at the University of Rochester. 11 The system is referred to as a 'Phase-Locked Interferometer.' In this instance one of the mirrors of the interferometer is oscillated so as to change the path length of one arm of the interferometer by about one-tenth of a 
wavelength. The oscillation creates an A.C. signal at a detector placed in the fringe pattern. By monitoring the frequency components present in the detector signal, the maximum and minimum of the fringe pattern are located very accurately ( 1/50th of a fringe spacing). By using a D.C. bias on the oscillating arm of the interferometer and galvanometers to scan the fringe pattern across the detector a very accurate contour map of the test sample can be generated. This interferometer is capable of functioning on a stand-alone basis with an $x-y$ plotter or oscilliscope as the output. It can also be interfaced with a computer for automated measurement and data analysis.

All but the digital and phase-locked methods have been applied to tnoire' systems. Both the techniques of digital interferometry and the Phase-Locked interferometry look promising in terms of accuracy and automation. The determining factor in the selection of which of these two systems to apply to moire' was the stand alone capability of the Phase-Locked system.

The first step in applying this technique to Moire fringe analysis is to decide on a method for oscillating the fringe pattern. The requirements for oscillation are:

1) the fringes should be able to oscillate at least $1 / 4$ of a fringe spacing. 
2) the oscillation frequency should be as high as possible to maximize data aquisition speed.

3) the oscillation should not cause distortion of the fringe pattern because of deformation of the object or grating.

4) should be as simple as possible.

In the case of a sinusoidal grating with both the object and observation point at infinity the following equation for the moire' fringe pattern is obtained:

$$
I=C\left[1+\frac{1}{2} \cos \frac{2 \pi z(\tan \alpha+\tan \beta)}{p}\right] .
$$

The argument of the cosine term contains four variables with which the fringe pattern can be varied; p-grating period, $z$-distance from the grating to the object surface, $\alpha$ - angle of incidence of the illuminating beam, and $\beta$ - viewing angle. The grating period $p$ is not a feasible choice since it is not possible to change the grating period without changing the grating. The grating-to-object distance, $z$, looks like a very good choice for many reasons. If $z$ is oscillated it could be expressed by

$$
z=z^{\prime}+\text { Asin } \omega t,
$$

where $z^{\prime}$ is the mean grating-to-object distance and Asinwt is the oscillating term. Substituting Eq. (39) 
into Eq. (36):

$$
I=C\left[1+\frac{1}{2} \cos \frac{2 \pi}{p}\left(z^{\prime}+A \sin \omega t\right)(\tan \alpha+\tan \beta)\right] .
$$

Letting

$$
\begin{gathered}
\frac{2 \pi}{\mathrm{P}}(\tan \alpha+\tan \beta)=k \\
I=C\left[1+\frac{1}{2} \operatorname{cosk}\left(z^{\prime}+A \sin \omega t\right)\right] .
\end{gathered}
$$

This is equivalent to the equation obtained for PhaseLocked interferometry ${ }^{12}$ :

$$
I=I_{1}+I_{2}+2 \sqrt{I_{1} I_{2}} \cos (\delta+A \sin \omega t) \quad .
$$

This equation is easily decomposed into its frequency components :

$$
\begin{gathered}
I=I_{1}+I_{2}+2 \sqrt{I_{1} I_{2}}\left\{\operatorname { c o s } \delta \left[J_{0}(A)+2 J_{2}(A) \cos 2 \omega t+\ldots\right.\right. \\
-\sin \delta\left[2 J_{1}(A) \sin \omega t+\ldots\right.
\end{gathered}
$$

Notice that at maximums and minimums of the fringe pattern $(\delta=n \pi)$ the mwt frequency components, where $m$ is an odd integer, all vanish. 
In order to oscillate $z$ either the grating or the object must be driven sinusoidally at as high a frequency as possible. Oscillating the object has a major drawback. For any type of driving system the maximum driving frequency is inversely proportional to the mass of the object. Therefore very massive objects are oscillated slowly or more power is required. The other alternative is to oscillate the grating. This could be done effectively up to the point where standing waves are set up in the grating causing distortion and possibly breakage but this would be at a much higher frequency than for a heavy object. Both of these methods bave two other serious drawbacks; first, if the fringe spacing is changed so must the driving amplitude A such that A Fringe Spacing/10; so large contour intervals mean large driving amplitudes and lower driving frequencies. Second, any system to drive even something as light as a grating couples considerable vibration to the surface on which it is mounted. Taking these problems into consideration, fringe pattern oscillation by driving either the object or the grating is feasible but it is not without some fairly serious problems.

The final alternatives, oscillating either $\alpha$ or $\beta$ can be considered together since they both enter Eq. (38) in the same way. Assume that $\alpha$ is varied sinosoidally such that

$$
\alpha=\alpha_{0}+A \sin \omega t
$$


where $\alpha_{0}$ is the bias angle and $A$ is the amplitude of oscillation. Substituting into Eq. (36)

$$
I=C\left[1+\frac{1}{2} \cos \left[\frac{2 \pi z}{p}\left(\tan \beta+\tan \left(\alpha_{0}+A \sin \omega t\right)\right)\right]\right] \quad .
$$

The magnitude of A must change the argument of the cosine by $\pi / 2$ (1/4 of a fringe) as sin( $\omega t)$ varies from -1 to 1 . Choosing $\beta=0^{\circ}$ and $\alpha_{0}=45^{\circ}$ (these values make the fringe spacing equal to the grating spacing since $\left.\left(\tan 0^{\circ}\right)+\left(\tan 45^{\circ}\right)=1\right)$ and substituting:

$$
I=C\left[1+\frac{i}{2} \cos \left[\frac{2 \pi z}{p}\left(\tan \left(45^{\circ}+A \sin \omega t\right)\right)\right]\right]
$$

To satisfy the above condition, then

$$
\frac{\pi}{2}=\frac{2 \pi z}{P}\left(\tan \left(A+45^{\circ}\right)-\tan \left(45^{\circ}-A\right)\right)
$$

which reduces to:

$$
\frac{\mathrm{p}}{4 z}=\tan \left(45^{\circ}+\mathrm{A}\right)-\tan \left(45^{\circ}-\mathrm{A}\right)
$$

From Eq. (46) it is apparent that the smaller $z$ gets the larger A must be. It is necessary to establish a reasonable lower limit on $z$. If $z \geq 10 p$ then the maximum 
value for $A$ occurs when $z=10 p$. Substituting this value for $z$ in Eq. (46) and solving for $A$ the result is found to be $A \simeq 0.5^{\circ}$. Half of a degree seems to be a reasonable scan angle. However, the field size must also be taken into account. If the diameter of the illuminating beam is $D$ and the diameter of the aperture of the scanning system is $d$, then the amplitude of the scanning system scan angle $\mathrm{A}^{\prime}=.5^{\circ}(\mathrm{D} / \mathrm{d})$. For example, a typical acousto-optic scanner has an aperture of about $3 \mathrm{~mm}$, if the system aperture is to be $10 \mathrm{~cm}$ then the deflection angle $A^{\prime}$ is $33^{\circ}$. These facts point out the trade-offs. An acousto or electro-optic deflector is the fastest available scanning system but since $A^{\prime}$ is limited to about $6^{\circ}$, D is limited to $2 \mathrm{~cm}$. A better choice seems to be a resonant scanner, at a resonant frequency of $2000 \mathrm{~Hz}$ with $\mathrm{d}=7 \mathrm{~mm} \quad \mathrm{~A}^{\prime}$ can be as large as $10^{\circ}$ permitting apertures of up to $20 \mathrm{~cm}$ in diameter.

This approach eliminates many of the problems present in the scheme which $z$ is oscillated. For example, vibration is no longer a problem since the mass of the oscillating mirror is so small. Also only one frequency and one amplitude of oscillation are required for a variety of combinations of grating periods and object sizes. The primary disadvantage of this system is the fact that the modulation amplitude varies almost linearly with $z$, this 
means that if the fringe oscillation amplitude (peak to peak) must lie in the range from $1 / 10$ to $1 / 2$ of a fringe then only fringes 4 through 20 satisfy this condition (where fringe number is $z / p$ ).

This system appears mechanically and optically feasible and it also satisfies the four requirements mentioned previously. It is now necessary to examine the frequency components of the intensity function (Eq. 44) to see if it is possible to uniquely determine the location of maxima and minima of the fringe pattern. Eq. (44) cannot be Fourier transformed in closed form and it must be done numerically. Figure $2-5$ shows the results of this numerical integration for the fundamental frequency and the first harmonic with

$$
\begin{aligned}
z & =(10 p) \text { to (1Ip) } \\
A & =.5^{\circ} \\
\alpha_{0} & =45^{\circ} \\
\beta & =0^{\circ}
\end{aligned}
$$

At the maxima and minima of the fringe pattern, the magnitude of the component at the fundamental falls to zero while the first harmonic component reaches its maximum. From this graph it is apparent that if a method could be devised to measure the magnitude of the component at the fundamental frequency it would be possible to accurately determine the location of the maxima and minima. 

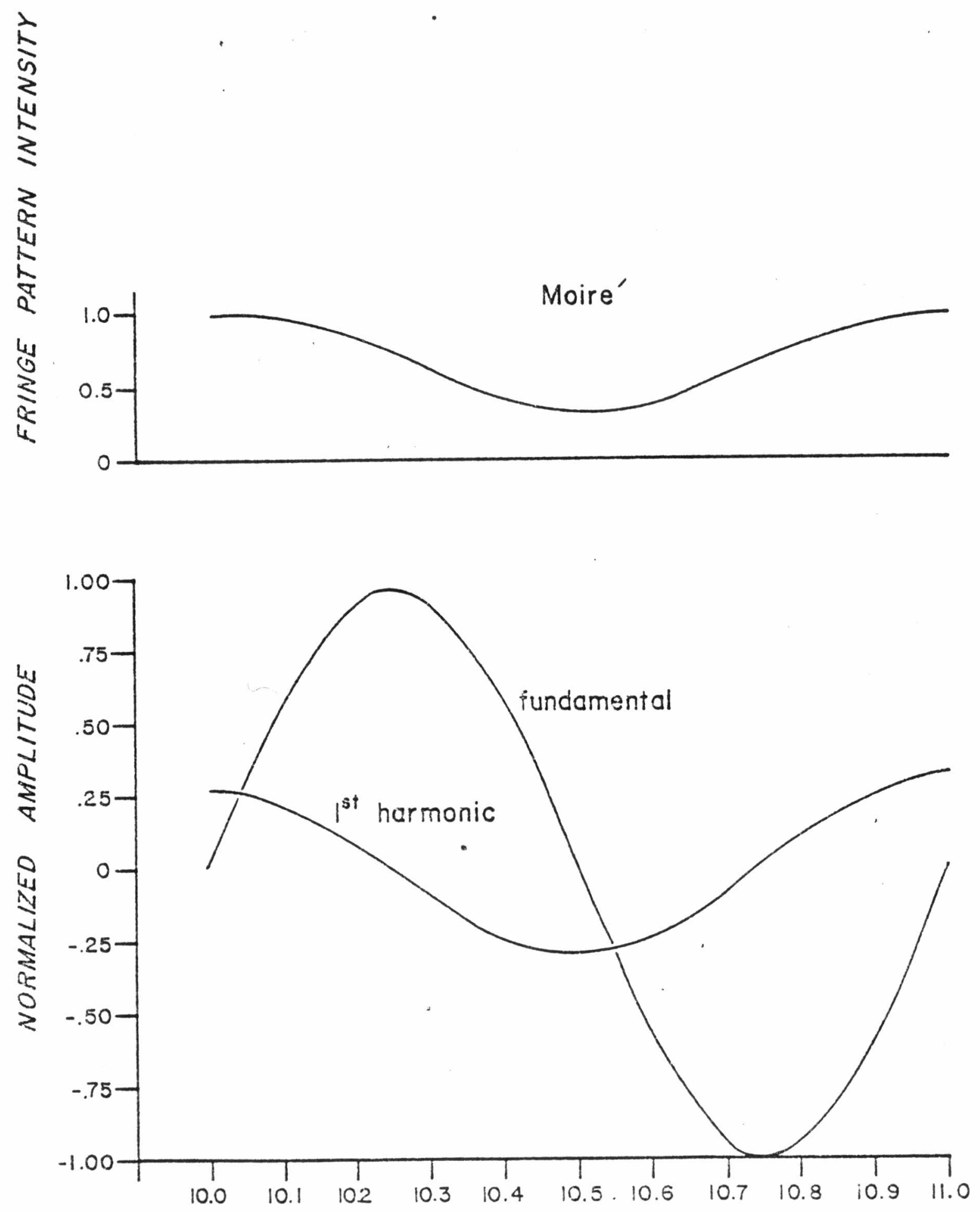

\section{DISTANCE FROM GRID}

Fundamental and First Harmonic Frequency Components of an Oscillating Moire'Fringe Pattern

Figure 2-5 
This is similar to phase-locked interferometry in which the amplitude of the fundamental frequency also goes to zero at maxima and minima of the fringe pattern (see Figure 2-6).

The similarity between the amplitude of the fundamental is illustrated in Figure 2-5. The only difference is a slight growth in the amplitude of the sinosoid as $z$ increases for the moire' system (Figure 2-7). Because of this similarity, the method of fringe detection used in phaselocked interferometry can be applied to the moire' system.

The concepts behind the implementation of phaselocked moire' fringe detection are illustrated in Figure $2-8$. Assume that the fringe pattern is being oscillated at a frequency $f$ by changing the angle of illumination as described previously. At time $t=0$ the fringe pattern is represented by $I_{1}$, a time $\frac{1}{4 f}$ later the fringe pattern has shifted to $I_{2}$ and after half a period the pattern has reached its maximum shift represented by $I_{3}$. A detector placed a position $\mathrm{P}_{1}$ will see a signal $\mathrm{S}_{1}$ consisting primarily of a component at the fundamental frequency $f$. If the detector is shifted towards the maximum to position $\mathrm{P}_{2}$ the detector signal is represented by $S_{2}$ which has temporal frequency components at both $f$ and 2f. Finally if the detector is centered on a maximum at $\mathrm{P}_{3}$ it will see a signal $\mathrm{S}_{3}$ which has primarily a $2 \mathrm{f}$ component and no component at the fundamental. 

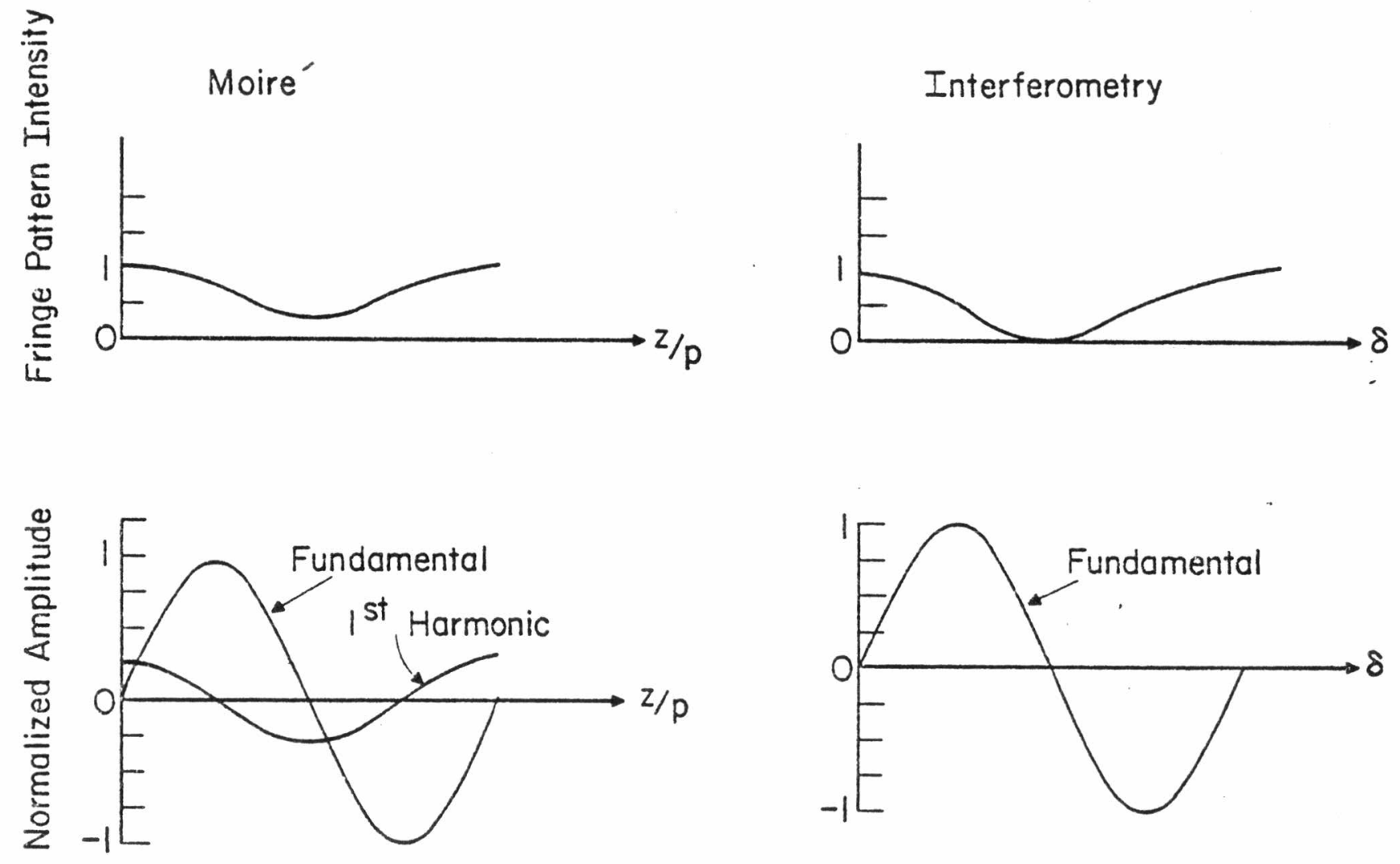

Comparison of Moire' and Interferometry

Figure 2-6 


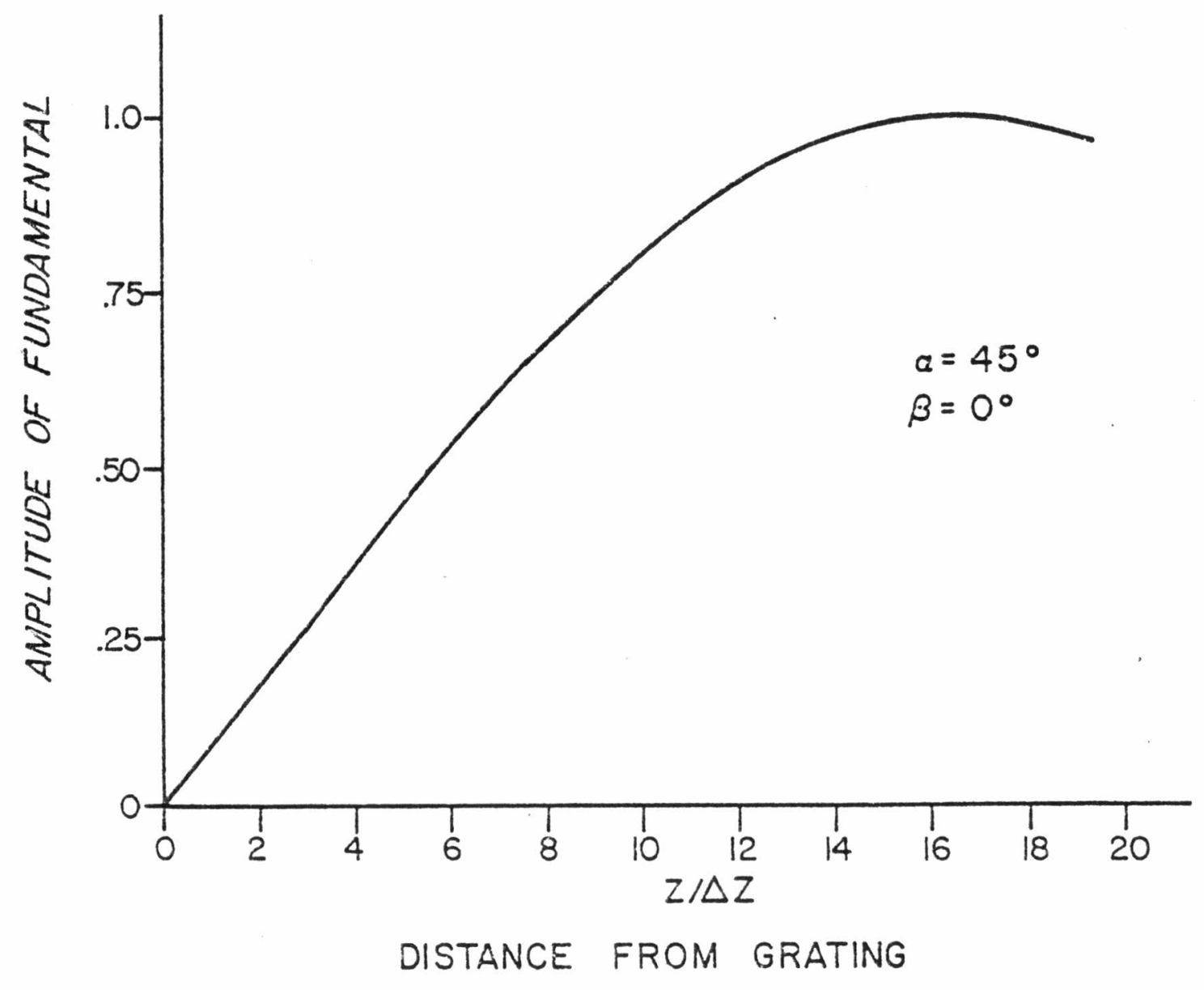

Amplitude of Fundamental Frequency

Component versus Fringe Number

Figure 2-7 


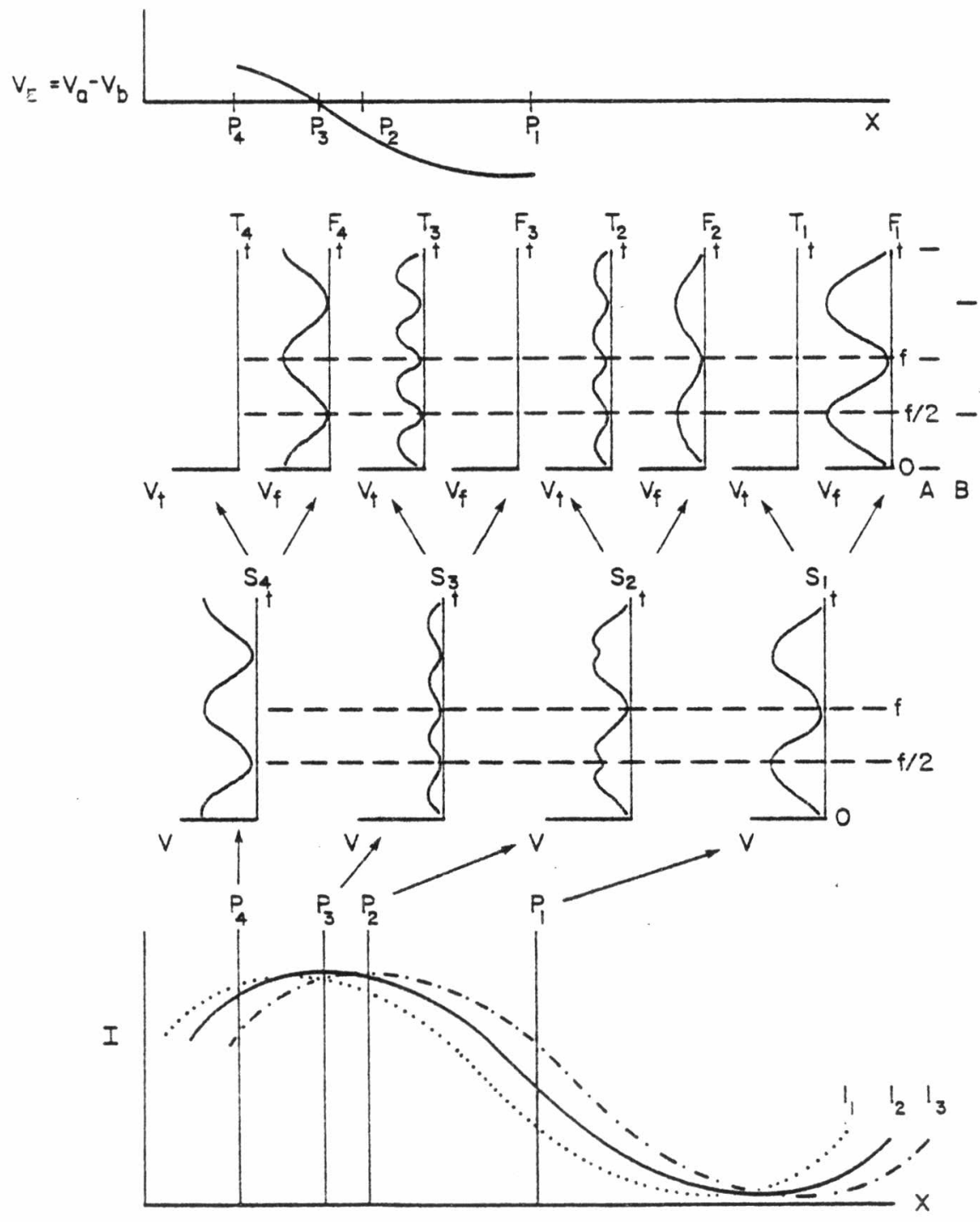

Concepts of Phase-Locked Moire'

Figure 2-8 
The detected signal is filtered to extract the components at $f$ and $2 f$, the outputs of these filters are represented by $F_{i}$ and $T_{i}$ respectively. As was stated previously a fringe maximum (or minimum) can be located by noting when the amplitude of the fundamental goes to zero. The magnitude of this signal is measured by sampling it twice each cycle at $t=0$ and $t=1 / 2 f$ represented by the points $A$ and $B$. This generates two voltages $V_{a}$ and $V_{b}$ and when they are differenced they give the error voltage $V_{E}$ which is representative of the distance to the nearest maximum (or minimum). The sign of $V_{E}$ indicates on which side of the fringe the detector is located. From Figure 2-5 it is seen that near a fringe maximum or minimum the magnitude of the fundamental and therefore $V_{E}$, is linearly proportional to the distance from the respective maximum(or minimum). To lock on a fringe $V_{E}$ is used to drive a motorized stage which changes the distance from the object to the grating. The stage is driven until the detector is centered on either a maximum(or minimum) of the fringe pattern at which point $\mathrm{V}_{\mathrm{E}}$ is zero and the motor stops.

To get a feeling for the accuracy of such a system refer to Figure 2-5. Notice that if the noise of the system corresponds to $1 / 4$ of the peak to peak amplitude of the fundamental then the accuracy to which the position of a fringe can be determined is about $\pm 1 / 20$ th of a fringe. 
If this noise can be reduced to $1 / 10$ of the peak to peak amplitude then the accuracy is improved to better than $\pm 1 / 50$ of a fringe. 
CHAPTER 3

\section{EXPERIMENTAL WORK}

\subsection{Design Concepts}

The theory of phase-locked moire'fringe analysis has been presented and it has been shown that phase-locked techniques make it possible to electronically locate the maxima and minima of the fringe pattern. In this chapter the design and operation of the instrument used to implement the concepts of phase-locked moire' fringe analysis for contouring diffusely reflecting surfaces is presented.

There are certain constraints imposed upon the system by the requirements placed on the final system and by the theoretical approach to the problem given in Chapter 2. The system is to be capable of contouring a surface to an accuracy of one micron over a $10 \times 10 \mathrm{~cm}$ square field. The system is to contour a surface either under computer control or as a real time system and do so as rapidly as possible. Because the system is to be versatile and able to contour objects of many different sizes, the performance of the instrument is to be invariant with respect to object size (within the $10 \times 10 \mathrm{~cm}$ aperture) and mass. Since the moire' theory closely 
parallels that of the Phase-Locked Interferometer the electronic processing requirements of the two devices are similar and the design of the circuitry is greatly simplified by applying the technology developed for the Phase-Locked system.

To contour an object a very simple approach is employed. The fringe pattern is imaged onto a detector and the output of this detector is processed by the modified PhaseLocked Interferometer Electronics (hence PLIM electronics), an error voltage $V_{E}$ ' is produced which is proportional to the amplitude of the fundamental frequency component in the detector signal. By mounting the object on a motorized translation stage driven in the $z$ direction by a d.c. motor, the error voltage can be amplified and used to drive the stage. If the detector is not located on the image of a fringe, then the stage is moved until a fringe appears on the detector at which point $\mathrm{V}_{\mathrm{E}}{ }^{\prime}$ goes to zero. (In order to avoid confusion it will be assumed that the electronics always drive the stage to locate the image of a minimum of the moire' pattern on the detector. The electronics are equally capable of locating a maximum by changing the sign of $\mathrm{V}_{E} \cdot$ ) Once the system 'locks' on a fringe the image is scanned across the detector by a mirror mounted on a galvanometer (Figure 3-1) and the stage is driven to keep the fringe on the detector. Since a surface of constant phase in the moire' pattern, always lies the same distance from the 
grating, the surface profile is obtained by measuring the stage position versus the position of the detector in the image. This contouring scheme has a very useful property which can be applied to the design of the imaging system. To increase the efficiency of the detector the imaging lens must have a low f-number and consequently a short depth of focus. To obtain the best image of the moire' pattern the depth of focus should be approximately equal to the distance between the grating and the object with the plane of best focus bisecting this distance. With this system since the distance from the grating to the point on the object being contoured is constant, the optimum focal plane for the moire pattern is stationary.' Since the grating to object distance is required to be much less than the distance from the grating to the imaging lens the depth of focus requirement is easily satisfied. Another feature of this contouring system is that it is not necessary to know the fringe spacing $\Delta z$. The contouring process has no dependence on fringe spacing since the detector is always locked on the same fringe. This is not to say that the fringe spacing is not important,for it is the fringe spacing which determines the vertical resolution of the system. This is a result of the fact that the electronics are capable of locking onto any fringe with the same accuracy expressed in terms of a fraction of the fringe spacing. This feature also 
eliminates the need to know some of the system parameters as accurately as would be necessary in a static system, the two of primary importance are the incident illumination angle $\alpha$ and the grating period $p$. Even though the grating period need not be accurately known it must remain constant across the grating. If it does not, the planes of the fringes are not flat and a systematic error in measurements made with that grating is introduced. It would seem that, since the incident illumination angle need not be known accurately, the same would be true of the viewing angle $\beta$ since they may be interchanged in the contouring equations without altering the results. The requirements on $\beta$ come from another part of the system, that is the imaging system. In order to maintain an orthogonal view of the object and minimize the depth of field problem, the viewing angle should be made equal to zero to within .01 radian. This accuracy guarantees that contouring errors introduced by non orthogonal viewing will be insignificant.

A requirement for contouring is that the position of the grating relative to the object be known. Alternately if the object is held stationary on its mounting stage, it is actually only necessary to know the position of the stage relative to the grating. There is also an accuracy requirement to measure the stage position with a resolution of one micron. This 
measurement is accomplished by the use of a Fabry-Perot interferometer which has one of its mirrors mounted on the grating mount and the second mirror on the motor driven stage. The interferometer is illuminated with some of the light from the laser used to illuminate the grating. If this light is from an argon laser operating at 0.5145 microns, then a transition from light to dark and back to light again will occur for every .2673 micron change in the stage to grating mount distance. By counting the fringes in the interferometer the position of the stage from some initial position can be determined to within one half of the wavelength of the incident light. This is well below the one micron accuracy required. 


\subsection{Optical System}

The optical system can be separated into 3 basic sections each performing independent functions;

1) the Fabry-Perot interferometer used to measure the stage motion, 2) the scanning system used to oscillate the angle of the illuminating beam on the grating and 3) the imaging system which images the moire' pattern and scans it across the detector. A detailed drawing of the system is given in Figure $3-1$ and a photograph is shown in Figure 3-2. The beam from the laser is first passed through a beam expander where it is expanded to about $10 \mathrm{~mm}$ in order to match the size of the mirror on the resonant scanner. The main expanded beam is incident on the scanner mirror at about $22 \frac{1}{2}^{\circ}$ causing the total deflection to be about $45^{\circ}$. After reflection from the scanner the beam is expanded once more up to its final size of $60 \mathrm{~mm}$ and is incident on the grating at an angle of incidence near $45^{\circ}$. The moire' pattern is imaged by a lens onto a detector after a reflection by a mirror mounted on a galvanometer. To scan the image the galvanometer is driven by a triangular wave. The amplitude determines the field size. 


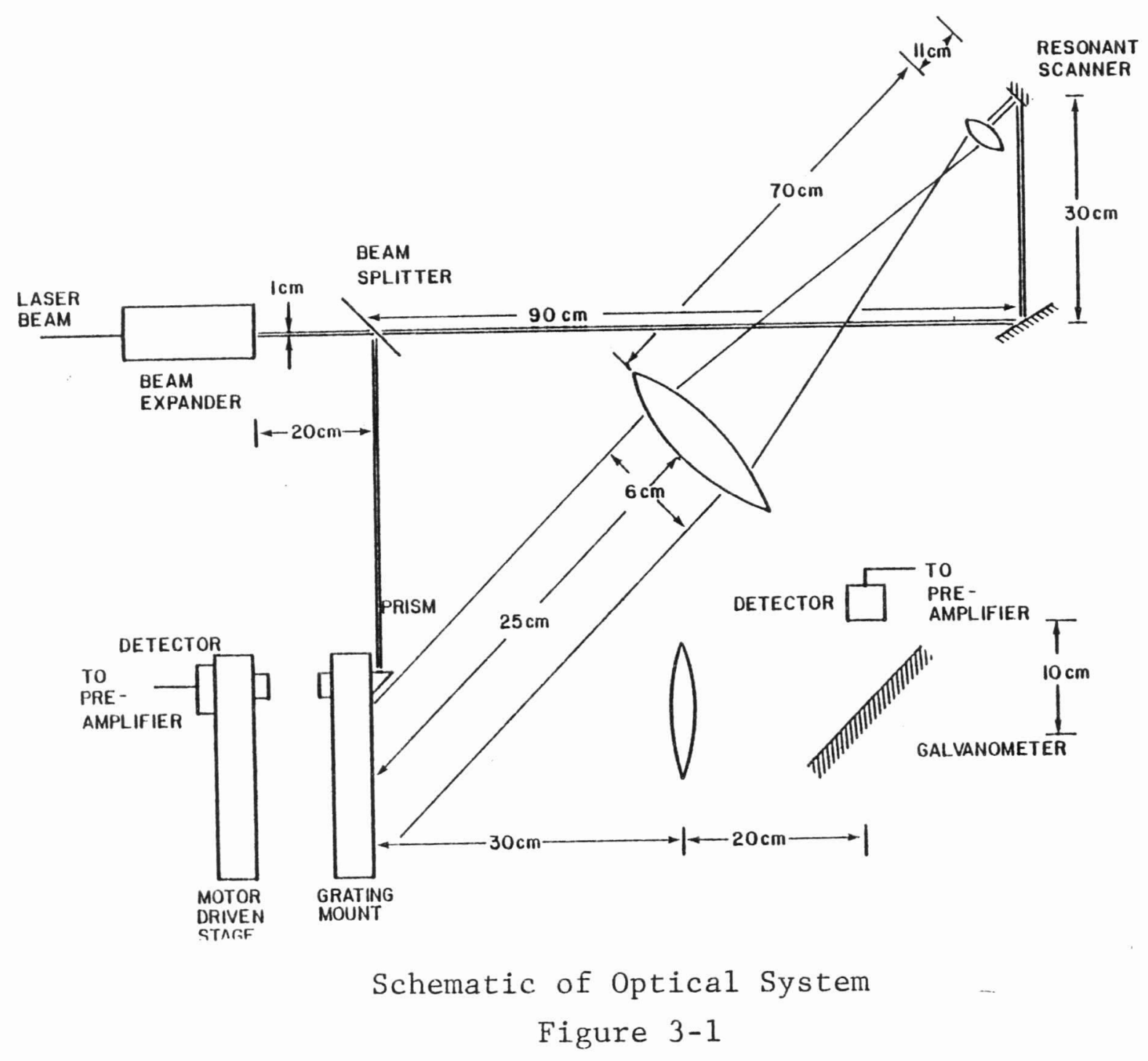




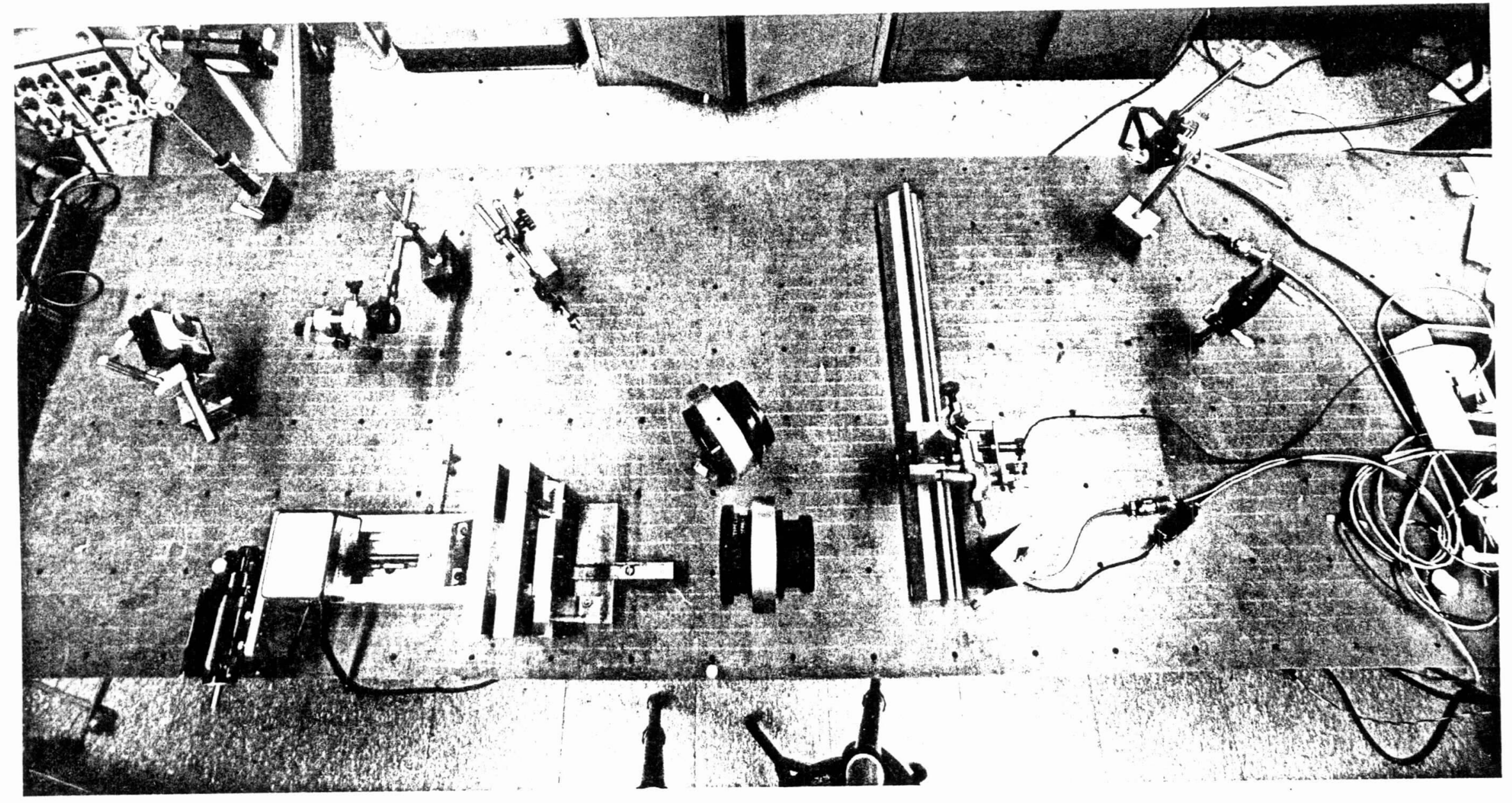

Photograph of Optical System

Figure 3-2 


\subsubsection{The Fabry-Perot}

The Fabry-Perot interferometer consists of two mirrors, one mounted on the grating holder and the second on the motor driven stage. The axis of the interferometer is defined by two $12 \mathrm{~mm}$ holes bored $25 \mathrm{~mm}$ from the edge of each piece. The mirrors are cemented over the holes on the inside surfaces of the two pieces with their uncoated side facing the part. They are $25 \mathrm{~mm}$ diameter mirrors which have been coated with a five layer dielectric coating with peak reflectance of about 50 percent at 0.600 microns.

The incident illumination from the beamsplitter in the main beam is parallel to the surface of the grating holder and about two millimeters from its outside surface passing directly over the hole for the Fabry-Perot. Cemented over the hole is a 45-45-90 prism with $1.25 \mathrm{~cm}$ square faces which reflects the beam into the cavity. The output emerges from the hole at the rear of the object stage. The interface with the electronics package is accomplished via a mounted detector which slides into the output hole. Alignment of the cavity is done by adjusting the angle of the grating mount with respect to the object stage by the use of three adjusting screws. The cavity must be aligned so that no more than one-half fringe is present across a $5 \mathrm{~mm}$ circular aperture at the center of the field. This assures a good output from the 
detector.

\subsubsection{The Scanning System}

The scanning system consists of a resonant scanner and a beam expander. The scanner is of the torsion bar type with a $10 \mathrm{~mm}$ diameter mirror mounted on one end of a stiff metal rod which has its other end clamped solidly in a mount, oscillation occurs about the longitudinal axis of the rod. The scanning system optics expands the $10 \mathrm{~mm}$ diameter collimated beam of light from the scanner by a factor of 6 to $60 \mathrm{~mm}$. The angular field must be large enough to accept an input beam oscillating $\pm 3^{\circ}$ to produce the required $\pm .5^{\circ}$ oscillation of the beam at the grating surface. Another design consideration is that lateral beam movement at the grating during oscillation must be minimized. If this is not done, then oscillation of the beam position causes intensity oscillations on the object because the intensity profile of the illuminating beam is not constant but Gaussian in cross-section. These intensity oscillations generate a signal at the detector of the same frequency as the signal generated by the oscillation of the moire' pattern possibly masking the desired signal completely. The scanning optics consists of a beam expander which ideally images the scaning mirror onto the grating. Because the grating and the scanning mirror are at about a $65^{\circ}$ angle to each other 
it is not possible to image one onto the other.

because of the small angle of oscillation at the grating it is sufficient. to image the center of the scanning mirror onto the center of the grating. A thin lens layout of the system is given in Figure 3-3.

Lenses $L_{1}$ and $L_{2}$ are chosen as a $10 \mathrm{~cm}$ focal length $3 \mathrm{~cm}$ diameter lens and a $60 \mathrm{~cm}$ focal length $6.5 \mathrm{~cm}$ diameter lens respectively. The selection of these two lenses sets $d$ at $70 \mathrm{~cm}$. The distance $t^{\prime}$ from $\mathrm{L}_{2}$ to the grating must be at least $25 \mathrm{~cm}$ in order that the grating can be viewed normally without obstruction by $\mathrm{L}_{2}$ and its mount. The selection of these parameters sets $t$ at $10.97 \mathrm{~cm}$.

\subsubsection{Imaging System}

The requirements on the imaging system are minimal. In fact some image degradation is desirable so that the system does not resolve the lines on the grating. Distortion in the imaging system is a problem. For precise measurements the distortion can be corrected in the data processing done by the computer.

The imaging system consists of a $15 \mathrm{~cm}$ focal length f/2.5 lens, a galvanometer with a $25 \mathrm{~mm}$ by $37 \mathrm{~mm}$ mirror with its longer direction mounted horizontally and a detector mounted behind a pinhole in the image plane of 


\section{IMAGING SYSTEM}

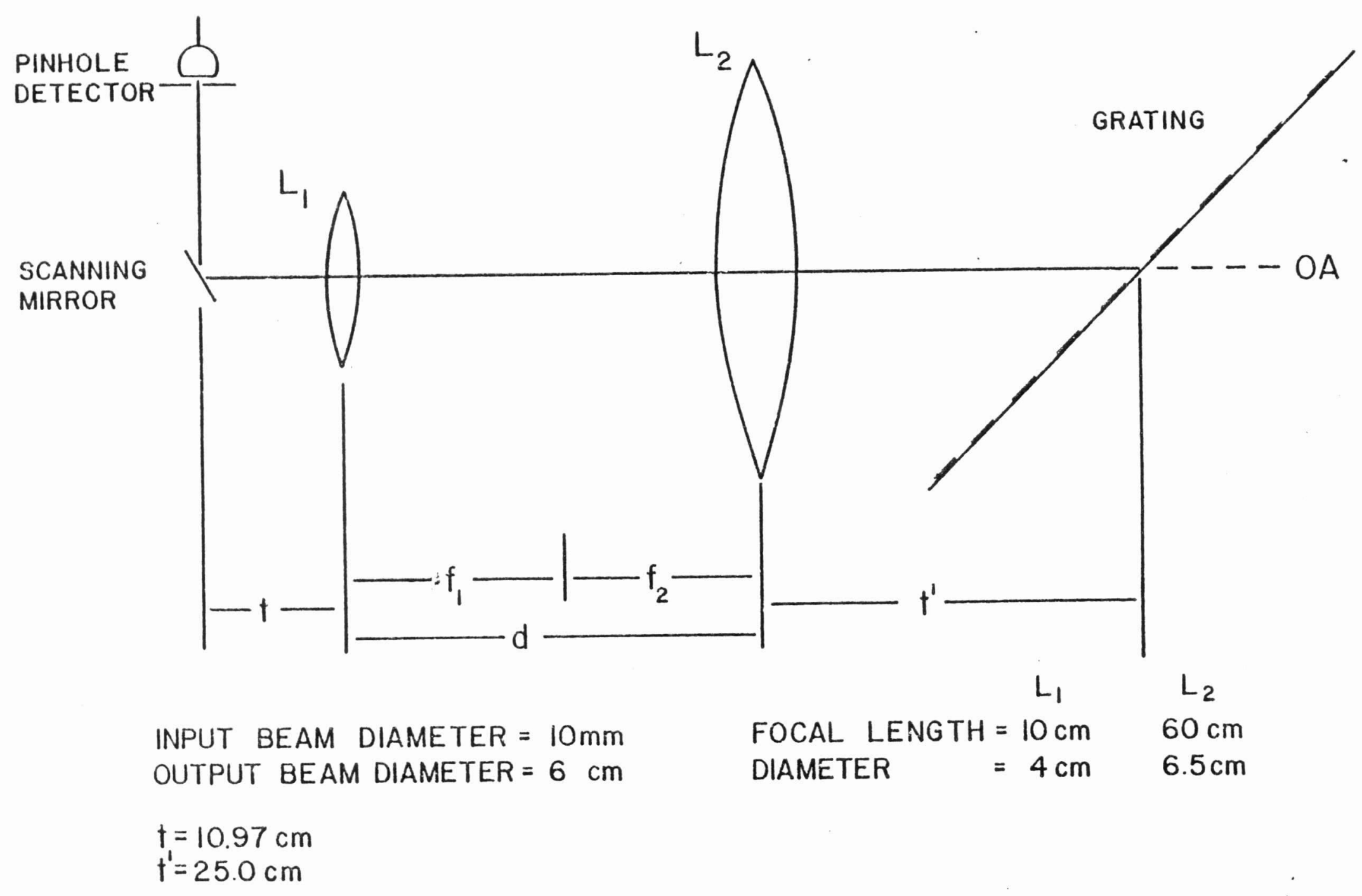


the 1ens. The arrangement of these components can be seen in Figure 3-1. The magnification of the image is adjusted by choosing the appropriate conjugate distances. 


\subsection{Electronics and Signal Processing}

The electronics controlling the moire' contouring are 12 an adapted version of the PLIM electronics and the majority of design concepts are similar. A general description of the electronics is given here and the circuit diagrams are located in Appendix $I$.

The functions performed by the electronics can be separated into 5 subsections; 1) the analog section which processes the signal from the moire' pattern and drives the motor to lock on a fringe, 2) the Fabry-Perot fringe counter which determines the position of the stage, 3) the image scanning electronics which control the scan of the galvanometer, 4) the digital section which interfaces the electronics with the computer and 5) an A-D converter which converts the various analog signals concerning galvanometer position, partial phase and signal strength. A block diagram of the processing sequence is given in Figure 3-4.

\subsubsection{Analog Processor}

The analog processing section of the electronic circuitry is the most complicated and the most important of any of the electronic sections. The performance of this section determines the contouring accuracy of the moire' system.

The modulated optical signal from the Moire pattern is detected by a photodiode placed in the image plane of 


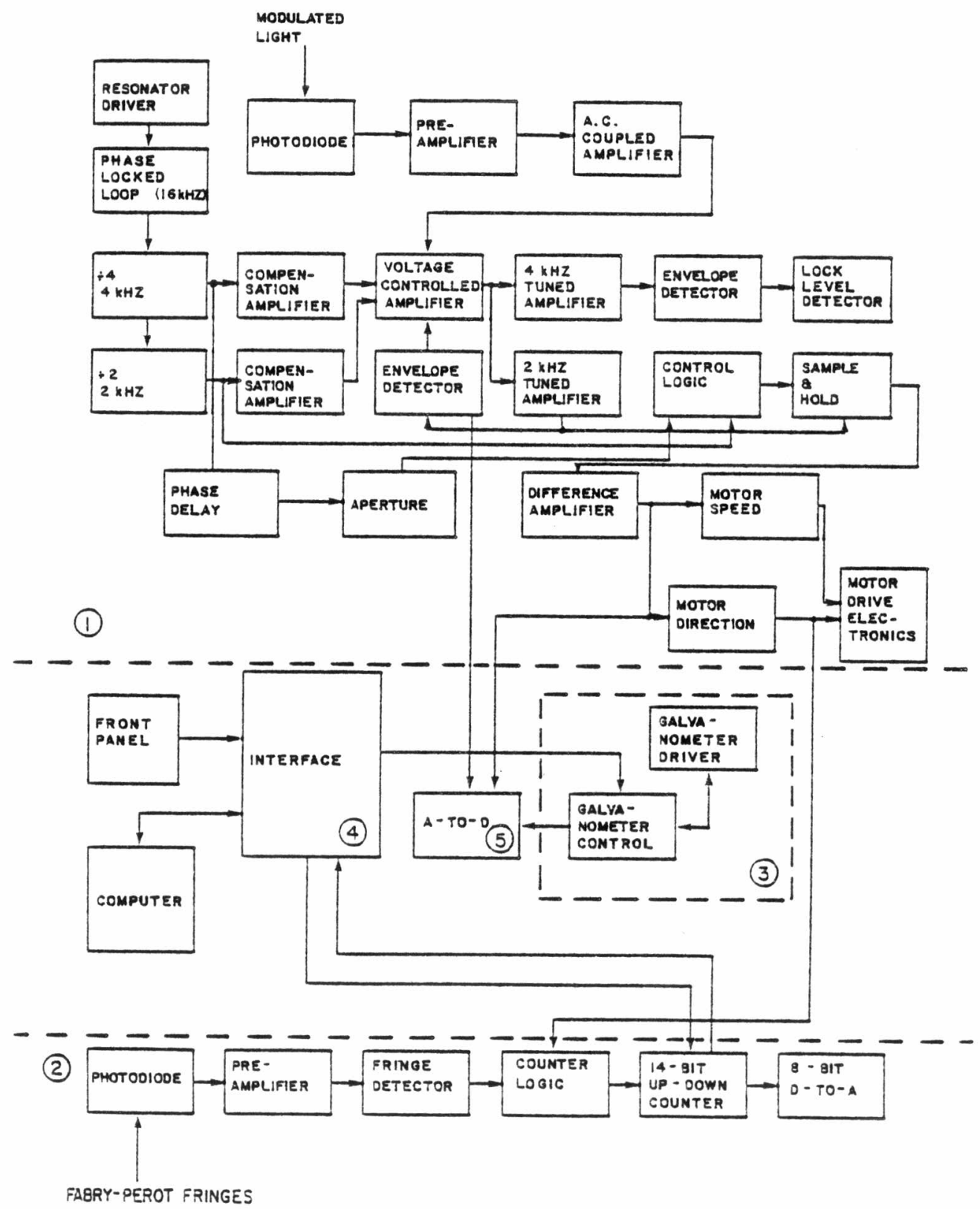

Block Diagram of Electronics

Figure 3-4 
the imaging system as described in section 3.2.3. This signal is then amplified by a high gain limited bandwidth amplifier which has its passband centered at $2 \mathrm{kHz}$ with lower and upper cutoff frequencies of about $100 \mathrm{~Hz}$ and $20 \mathrm{kHz}$. The amplified detector signal is fed into a voltage-controlled amplifier. This functions to keep the desired signal level constant independent of changes in image intensity (this is simply an automatic gain control circuit.) The controlling voltage for the VCA is derived from an envelope detector which detects the amplitude of the $2 \mathrm{kHz}$ portion of the signal.

The output of the VCA is split and fed into two high $Q$, tuned amplifiers, one tuned to $2 \mathrm{kHz}$ and the second tuned to $4 \mathrm{kHz}$. The output of the $4 \mathrm{kHz}$ tuned amplifier is rectified and integrated producing a signal which is proportional to the magnitude of the first harmonic. A level detector is used to examine this signal. The level indicates when the detector is at a minimum of a fringe pattern (see Fig. 2-6.) The signal from the $2 \mathrm{kHz}$ tuned amplifier is also processed by a level detector which controls the VCA. The output of the $2 \mathrm{kHz}$ tuned amplifier is the signal from which the error voltage $V_{E}$ ' is derived. This is done by sampling the filtered signal at its maximum and minimum with a sample and hold circuit. The two voltages obtained are then differenced in a differential amplifier. The output is the error voltage $V_{E}{ }^{\prime}$. Because of the 
operation of the gain control circuitry, the error voltage $\mathrm{V}_{\mathrm{E}}$ ' is not linearly dependent on the error voltage $V_{E}$ except near fringe maximum and minimum.

The next step is to use the error voltage to drive the motor. To do this, the error voltage is fed into a level detector and a rectifier generating two signals the sign of $V_{E}{ }^{\prime}$ and its absolute value, respectively. The output of the level detector is used to control a double throw 4 pole relay which switches the polarity of the voltage on the field coil of the motor. The magnitude of $\mathrm{V}_{\mathrm{E}}{ }^{\prime}$ is amplified from 0 - 10 volts at the output of the rectifier to 0-100 volts on the armature coil of the motor. This completes the feedback loop for locking the detector onto a fringe. Whether the system chooses to lock on a maximum or minimum is determined by the sign of the product of the field coil voltage and the error voltage and is switchable by means of a manual switch in the field coil circuit which controls the polarity of the field coil.

Two parts of the analog circuit section of Figure 3-4 are important for the proper operation of the system. The first snychronizes the operation of the sample and hold with the optical signal. The second generates compensation signals which can be mixed with the incoming signal at the VCA in order to cancel any background signals at the frequencies of interest. Both of these functions 
are synchronized to the resonator through its driving electronics. This is done through a TTL compatible signal generated within the driver by a level detector fed with a signal from a transducer in the resontant scanner. The TTL signal is used as a reference signal by a phase-locked 1oop which oscillates at $16 \mathrm{kHz}$. The $16 \mathrm{kHz}$ signal is divided by four to produce the reference signal for the $4 \mathrm{kHz}$ compensation amplifier and divided by eight to produce a reference signal at $2 \mathrm{kHz}$. This signal is used for the $2 \mathrm{kHz}$ compensation amplifier and as a reference for the pulse generator which is used to trigger the sample and hold circuits.

\subsubsection{Fringe Counter}

The fringe counter keeps an accurate record of the position of the stage relative to its initial position. To do this, the counter must detect every fringe which passes by the detector and note whether the fringe signifies increasing or decreasing phase. The counter must also be immune to spurious noise generated by vibrations of the stage and grating mount. The circuitry used to implement the fringe counter is relatively simple and is sketched out in block form in Figure 3-4.

The intensity variations in the output of the FabryPerot interferometer are detected by a silicon photodiode and amplified to a five to ten volt amplitude level by 
the preamplifier. This signal is processed by a zerocrossing detector to generate a TTL signal used as the counting pulse. The level detector has been designed with a large hysteresis loop of about one half of the signal amplitude to eliminate any false counts due to vibrations. The count pulses are finally directed to either the count up or count down input on the counter depending on the direction which the stage is being driven. The counter itself is a 14 bit binary up-down counter allowing it to keep track of about $4 \mathrm{~mm}$ of stage movement. To avoid overflow and underflow problems when taking data, the computer sets the counter to the middle of its range. In order to provide an analog signal proportional to the stage position the lower order eight bits from the counter are fed into a digital to analog converter.

\subsubsection{Image Scanning Electronics}

The image scanning electronics drive the galvanometer in a periodic scan and output a signal which accurateIy represents the position of the mirror. This section of the electronics is designed to operate completely independently of all other sections.

The electronics are separated into two parts, the galvanometer control and the galvanometer driver. The control contains a signal generator which produces a triangular waveform with variable amplitude, offset and 
frequency. These are adjustable by controls on the front panel of the instrument. The control also allows the scan frequency to be set to one of 3 predetermined values by a command from the computer. Another option which has been designed into the galvanometer control is the provision for generating a signal to drive a second galvanometer producing a raster type scan with 16 to 256 lines over a variable sized aperture.

The galvanometer driver takes the signal from the control and amplifies it in order to drive the galvanometer. The driver also contains a feedback system which accurately controls the position of the galvanometer mirror through the use of a position transducer built into the galvanometer.

\subsubsection{Computer Interface}

The computer interface (CI) allows the computer to communicate with the system, permits the computer to set up the conditions necessary to initiate a scan and supplies the data and status information required by the computer.

The interface consists of two ports, an input port and an output port each with 16 bits. The input port receives a 16 bit word from the computer and the bits are assigned as follows: 
$\underline{B i t}$

Function

$0 \quad$ Multiplex bit

$1 \quad x$ galvo on $=1$ front panel $=0$

$2 \quad \mathrm{y}$ galvo on $=1$, front panel $=0$

3 Initiate stage position counter

4 Number of lines in scan-00, front panel;

501,16 lines; 10,32 lines; 11,64 lines

$6 \quad$ Period of scan - 00 , front panel

7 01, medium speed;10, slow speed;11, high speed

8 not used

$9 \quad$ clear galvo counters

Galvo count up

Reset bit

not used

not used

not used

Bits 2,4,5 and 9-12 are used for controlling a second galvanometer for use in a two dimensional scan and are not currently in use. Bit zero is a multiplex bit and is used to control which of the two data words are read. If the bit is set to logical one, then the data present at the output port is the position of the stage. If the bit is set to zero, then a number of status bits are read. Bit 1 is used to switch on the galvanometer if it was not turned on at the front panel. If this bit is set to one, then the galvanometer switch on the front panel is overridden. The galvanometer remains on until the bit is reset to zero at which point the front panel switch is reactivated. Bit 3 is used to preset the fringe counter, by changing the bit from logical 1 to 0 and back to 1 again. 
Bits 6 and 7 set the scan rate to one of three preset values. If they are set to anything but $\emptyset \emptyset$ the scan rate adjustment on the front panel is overidden and the computer selected value is used.

The digital output of the phase locked electronics is composed of two 16 bit words which are multiplexed out through a single 16 bit output port by the multiplex bit sent from the computer. If this bit is zero, then a word consisting only of status bits is read. At the present time only two of these bits are activated. Bit $\emptyset$ is equal to the multiplex bit sent by the computer and is used to verify the word being read. The only other activated bit is 3. This bit is set equal to zero if the signal from the pattern is sufficient for proper analysis and one otherwise. The second 16 bit word which is read when the multiplex bit is one,contains the value of the stage position counter and two status bits. Bit 0 again contains the multiplex bit which should now be a logical 1 . Bit 1 , the other status bit is zero if the data is in transition and one if the data is stable. The fourteen highest order bits contain the value of the counter.

\subsubsection{Analog to Digital Converter}

The Analog to Digital Converter is the second half of the link from the phase-locking electronics to the computer. This unit has four analog input channels which 
are sampled sequentially at rates of up to approximately 1,000 times per second. The output of the A-to-D is a single 16-bit word coded as follows:

$\underline{B i t}$

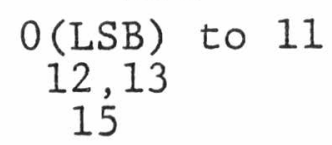

Function

12 bit analog word channel number $(0,3)$ data change

Each of the four channels is assigned to a different analog output from the modified PLIM electronics. Channel $\emptyset$ is assigned to the position signal from the galvanometer. Channel 1 is not being used in the current system. This channel has been reserved for a second galvanometer when two dimensional scanning of the object is employed. Channel 2 is assigned to the error voltage $V_{E}^{\prime}$. Lastly channel 3 is assigned to the controlling voltage of the VCA in the automatic gain control circuit. 


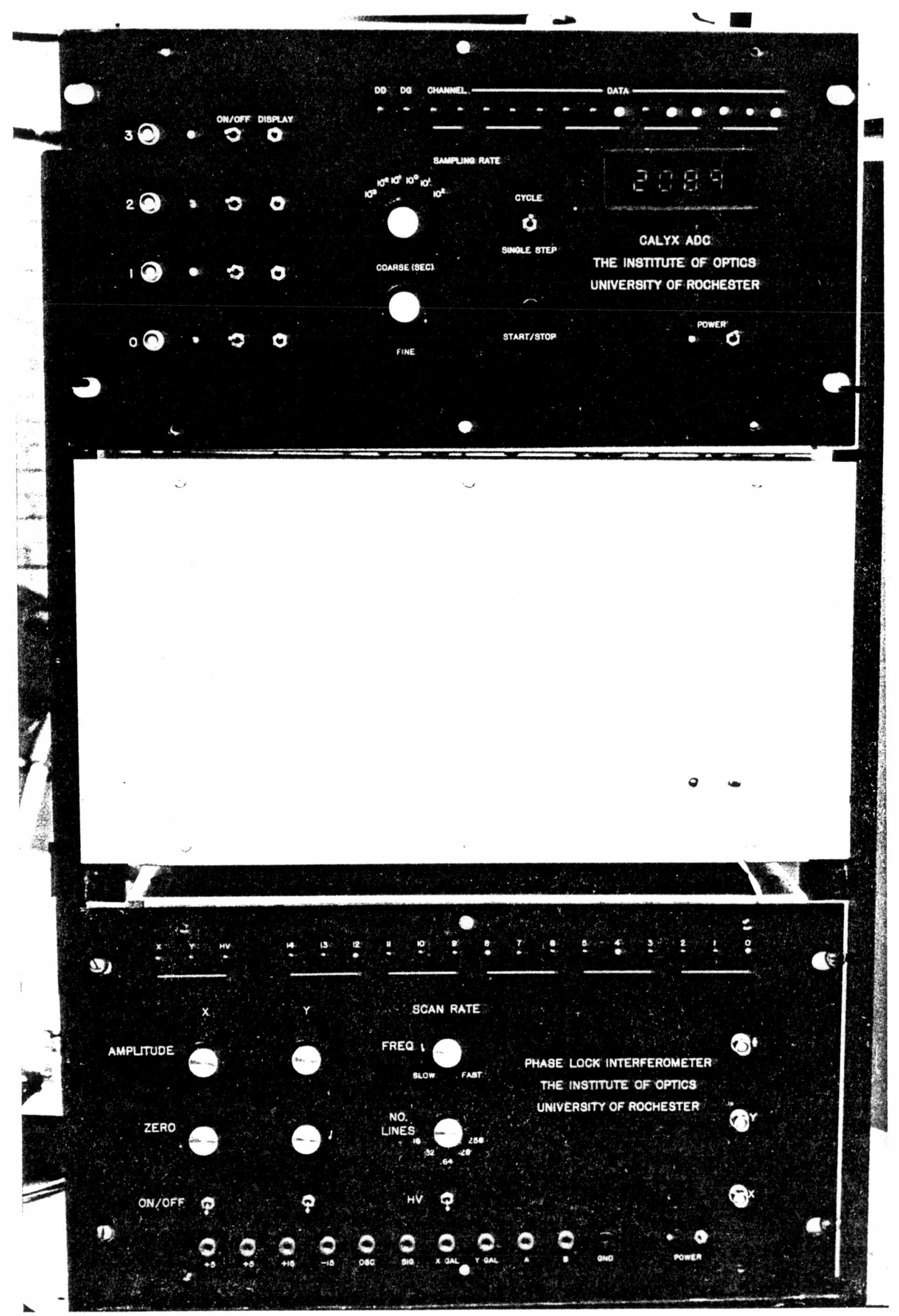

Photograph of Electronics

Figure $3-5$ 


\subsection{Data Processing}

The experimental system is interfaced with a Data General Nova 2/10 Computer equipped with $32 \mathrm{k}$ words of core and a 5602 I/O unit. The data acquisition program, MOIRE1 is quite extensive as it was originally written for a production version of the Phase-Locked Interferometer. The interferometer version of this program was altered in details only to make it compatable with the moire' system. Detailed error checks and two dimensional scan capabilities remain in the program but have been bypassed for MOIRE1. These capabilities remain available for use in future versions of this system.

In the current version of MOIREl only the multiple $\mathrm{x}$-scan capability of the program is in use. This section of the program measures the surface profile along a single line across the object. The output, which was designed primarily to characterize spherical surfaces, consists of the calculated values of surface power, tilt, asphericity, peak to peak roughness and r.m.s. roughness. Also the profile can be plotted on an $x-y$ plotter, stored in a disk file or printed out in table form.

The multiple $\mathrm{x}$-scan portion of the program performs five functions. It reads in data, processes this data, does a curve fit and plots the surface profile. The first part of the program reads in five data words for each field position. These five words are the value of the stage 
position counter and the four channels of the A to D. The data processing section takes three of these five words, the error voltage, the automatic gain control voltage and the fringe count and combines them to form the contour height information. This combined data along with the position of the galvanometer is scaled to real values, the $x$-position in millimeters and the surface height in microns. The third section of the program does a curve fit to the scaled data. This curve fit generates the values for power, asphere, tilt and roughness mentioned previously. The final portion of the multiple $x$-scan program plots the scaled data providing a hard-copy record of the surface profile.

For a more detailed description of the computer program and a listing of the computer code see Appendix 2. A complete description of the capabilities of the original program for the phase-locked interferometer can be found in the manual for the Phase Locked Interference Microscope. ${ }^{17}$ 
CHAPTER 4

\section{RESULTS}

This chapter examines the operation of the phaselocked moire' system. The first section deals with the operation of the optical system and the electronics. The second section presents the results of surface measurements done with the system and an analysis of its performance. 


\subsection{Optical System and Electronics}

The optical system performs well, but it has two sections which are very critical in terms of alignment. The most sensitive section of the optical system is the beam expander following the resonant scanner. Lateral motion of the beam on the object surface due to the resonant scanning system is a serious problem. If there is the slightest lateral movement of the beam, a signal at $2 \mathrm{kHz}$ is generated because the cross section of the beam is not uniform in irradiance. The magnitude of this signal is usually equal to or greater than the magnitude of the desired signal thereby masking it completely. This problem can only be eliminated by accurately imaging the center of the scanning mirror onto the center of the grating surface. It is found experimentally that this requires the first lens of the beam expander to be located with better than one millimeter accuracy.

The imaging system is also very sensitive to alignment and focus. For best operation the imaging lens is aligned by autocollimation from the grating surface. The lens is followed by the galvanometer which is set at $45^{\circ}$ to the optical axis. Finally the detector is located in the image plane of the fringe to be used for contouring. 
The analog signal processing which takes the detector signal and converts it to an error voltage did not work as well as had been expected. This section did a fine job digging the optical $2 \mathrm{kHz}$ signal out of the optical background noise. In fact the rejection of $120 \mathrm{~Hz}$ was so great that the system will work even with the room lights on. The problem is a fluctuation in the amplitude of the $2 \mathrm{kHz}$ filtered signal when all possible optical sources of noise are removed. This fluctuation causes a like fluctuation in the error voltage $V_{E}{ }^{\prime}$. Filtering the error voltage over a period of about 20 cycles (.01 sec) eliminated the majority of the noise but there is still some left at lower frequencies. It is this noise which appears to be the limiting factor for the minimum optical signal strength required for processing.

The Fabry-Perot fringe counter has excellent accuracy. When correctly adjusted, it is not subject to error due to room noise or motor vibrations. Extra counts due to noise were only recorded when the optical table was struck sharply. The only problem with the counter occurs if the motor suddenly changes direction. This occurs because there is a lag between the time the motor changes direction and the time the stage changes direction. Since the direction of the counter is determined by the direction of the motor, the count direction changes before the stage direction does thereby causing a counting error. 
The operation of the motorized stage is not as smooth as it could be due to the properties of the motor drive. The motor requires a minimum initial voltage on the armature to start rotation. Once rotation begins the voltage needed to sustain it is much less. The combination of these two factors causes the motor to move in steps of one-tenth to one-quarter of a fringe spacing. These steps can be smoothed by adding the error voltage as a correction. This is easily done in software since the error voltage is one of the values available from the A to D. The stepping also limits the speed of a scan because the jumpy motion causes the system to slip from one fringe to the next during a scan. 


\subsection{Measurements and analysis}

Measurements were made with the phase-locked Moire system while interfaced with the Nova computer. This system is the same as that already described but with two exceptions. First, the angle of illumination could not be made equal to $45^{\circ}$ due to space limitations on the optical table. The angle which was used is $32.5^{\circ} \pm 1^{\circ}$. Secondly a HeNe laser had to be used to illuminate the Fabry-Perot cavity on the grating mount while the grating illumination was provided by an Argon ion laser operating at $.5145 \mu$.

Surface measurements were first done on a ground glass surface which had been painted white. The purpose of the paint was to maximize the diffuse reflectance of the surface in order to form high contrast fringes. The electronics were checked out using the analog output capability of the system and a dual trace oscilloscope. Contours can be observed on the oscilloscope if the galvanometer position is used as the horizontal input and the output of the D-to-A on the fringe counter is used as the vertical input.

Once the instrument is adjusted and the contour on the oscilloscope looks good, the computer is used to take data. The first measurements, recorded the output of the fringe counter versus the galvanometer position. Since the motor drives the stage in steps, a graph of the data 
had the form of a staircase. An example of this staircase phenomena is illustrated in Figure 4-1. This graph represents the surface profile of a spherical glass surface.

The next step was to scale the error voltage and add it to the fringe count in order to smooth out the steps. The program, MOIRE1, has the capability of converting the error voltage to a number of fringe counts and adding it to the value of the stage position counter. The equation used to perform the converstion is

$$
(\mathrm{VE}+\mathrm{VO}) \mathrm{VF}=\mathrm{FC}
$$

where $V E$ is the error voltage, $V O$ is the offset voltage, $\mathrm{VF}$ is the number of fringes per volt and $\mathrm{FC}$ is the fringe count correction. A number of measurements were made and the optimal values were found to be .85 volts for the offset voltage and 25 fringes/volt for the scaling factor. Figure 4-2 illustrates the effect of this correction. This contour was made from the same surface as Figure 4-1 but with the addition of the error voltage as a correction. Once the contours had been smoothed out the system was checked for repeatability and retrace accuracy. Retrace accuracy is the ability of the instrument to produce the same contour irrespective of the direction of the galvanometer scan. This was done by making six consecutive measurements of the same painted glass surface over a 


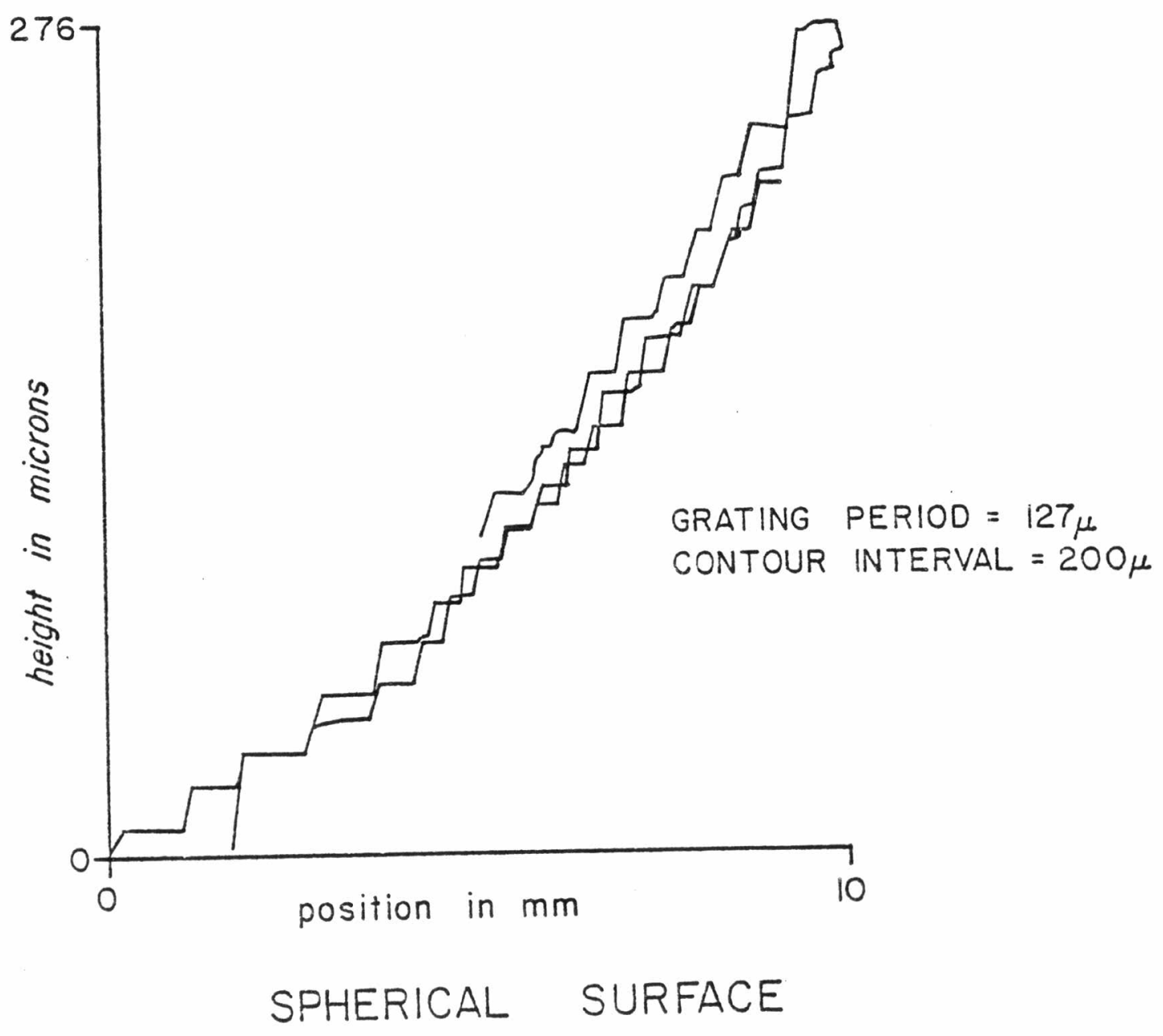

Contour of Spherical Surface without

Error Voltage Correction

Figure 4-1 


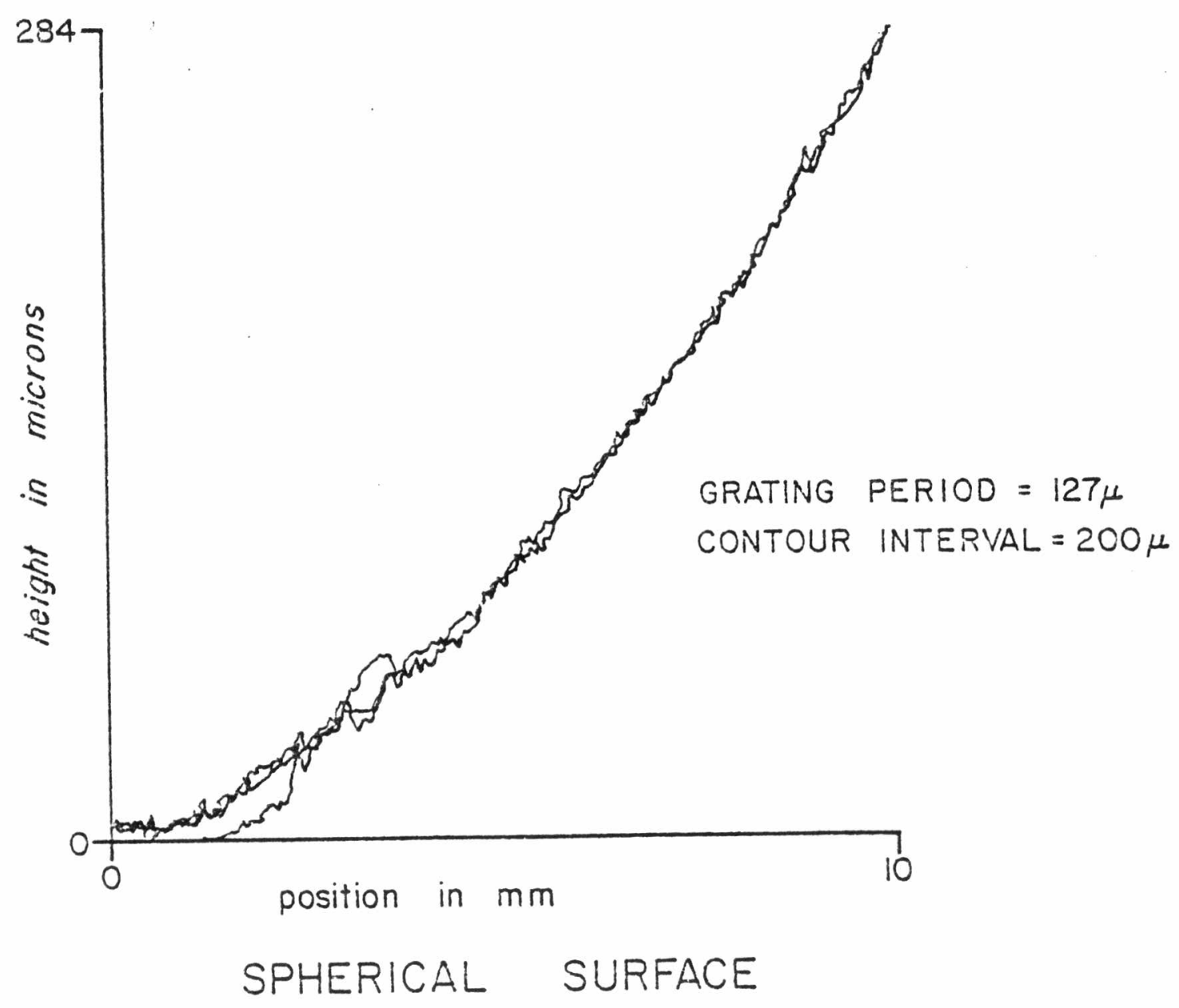

Contour of Spherical Surface with

Error Voltage Correction

Figure $4-2$ 
time long enough so the galvanometer does a complete scan in each direction. Three values were measured from each graph. These values were; 1) the maximum retrace error, 2) the typical retrace error representing the average maximum error excluding the point at which the maximum error occurred and 3) the height of the graph at half field. These measurements were made with an image magnification equal to one, a pinhole size of one millimeter and a scanning speed of .1 centimeters per second.

The average maximum error for this group was $25 \mu$. The grating used had a period of $127 \mu$ which converts to a contour interval of $200 \mu$ when the angle of incidence is $32.5^{\circ}$. In terms of a fraction of the contouring interval the maximum error was one-eighth of a fringe. The maximum error, $E_{\max }$ observed was $32 \mu$. The average typical error, $E_{\text {typ }}$ observed was $11 \mu$ with a maximum $E_{\text {typ }}$ of $12 \mu$ corresponding to slightly more than one-twentieth of the contouring interval. The average height at half field was $94.0 \mu$ with values between $89.2 \mu$ and $102.8 \mu$. If the one value of $102.8 \mu$ is thrown out then the remaining measurements all lie in a range of $5 \mu$. The graph in which the half field height is 102.8 is atypical due to an inconsistent downward dip near the $z=0$ axis which is not present in the other graphs. One of the contours generated for this test is shown in Figure 4-3. The point of maximum error clearly occurs in the upper left hand corner of the graph. This 


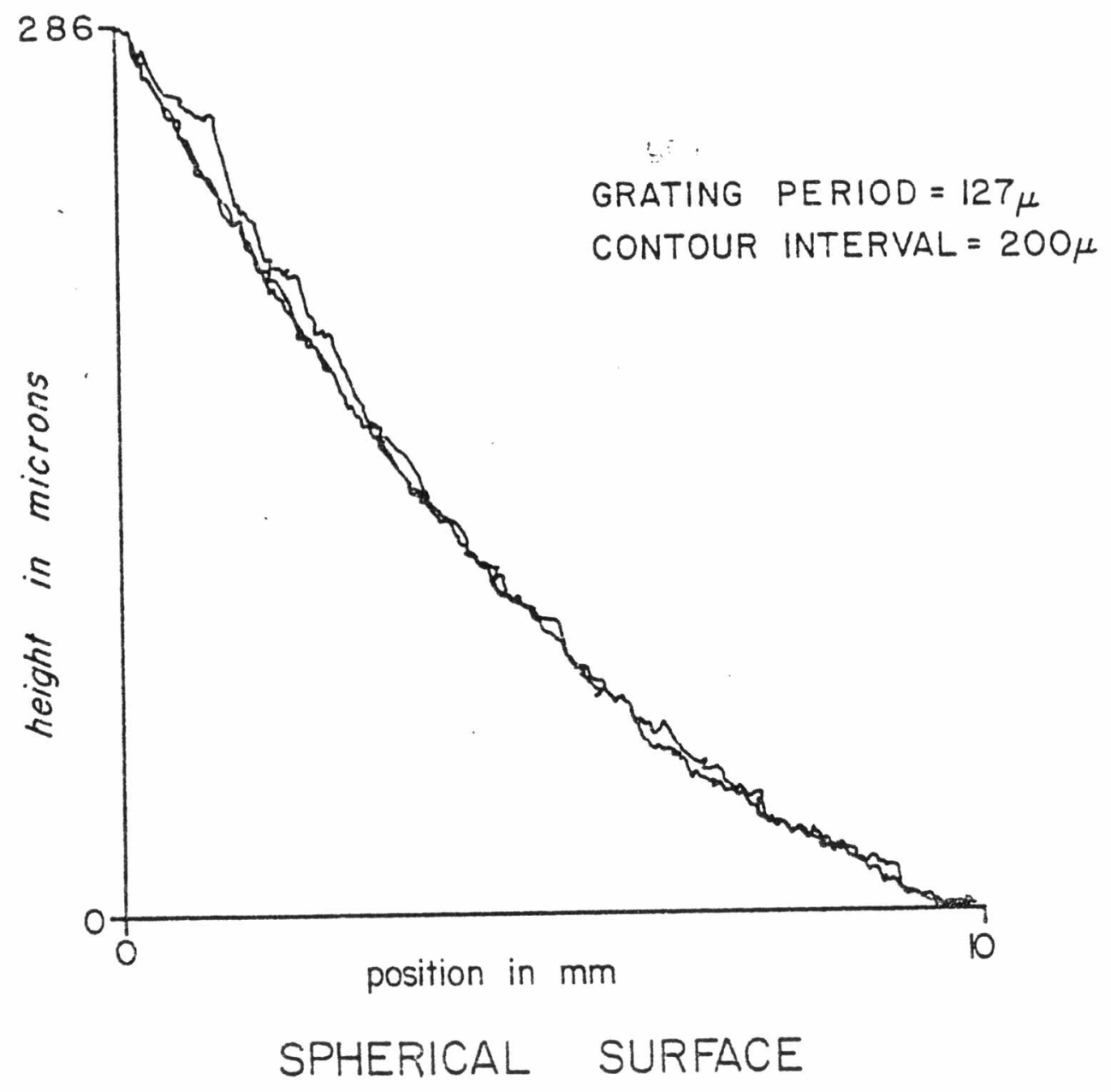

Example of Contour used to Check Retrace Accuracy

Figure 4-3 
is typical of the entire set and is due to the fact that some of the steps are not completely eliminated by the addition of the error voltage. This error can be corrected in one of two ways. The first and most desirable method is to smooth out the motor drive thus eliminating the steps entirely. Another possible solution is to write a subroutine for MOIREl which scales the error voltage separately for each step by matching the data before and after each step. If this problem is corrected then the remaining retrace error is represented by $E_{\text {typ }}$ and is primarily due to signal noise in the detector and preamplifier.

The repeatability of the half-field height measurement looks promising since it is consistent to within $1 / 40$ th of the contouring interval. These results indicate that it should be possible to obtain repeatable accuracies of $\Delta z / 40$ with a modification of the motor drive and some work on the analog signal processing.

An example of resolution of surface structure less than $10 \mu$ in amplitude is exhibited in Figure 4-4. The contour interval in this case was $200 \mu$. This graph is the surface profile of another spherical surface which has also been painted white. The structure at the bottom of the graph appears on five consecutive measurements. Examination of the object shows the structure to be due to a defect in the paint. 


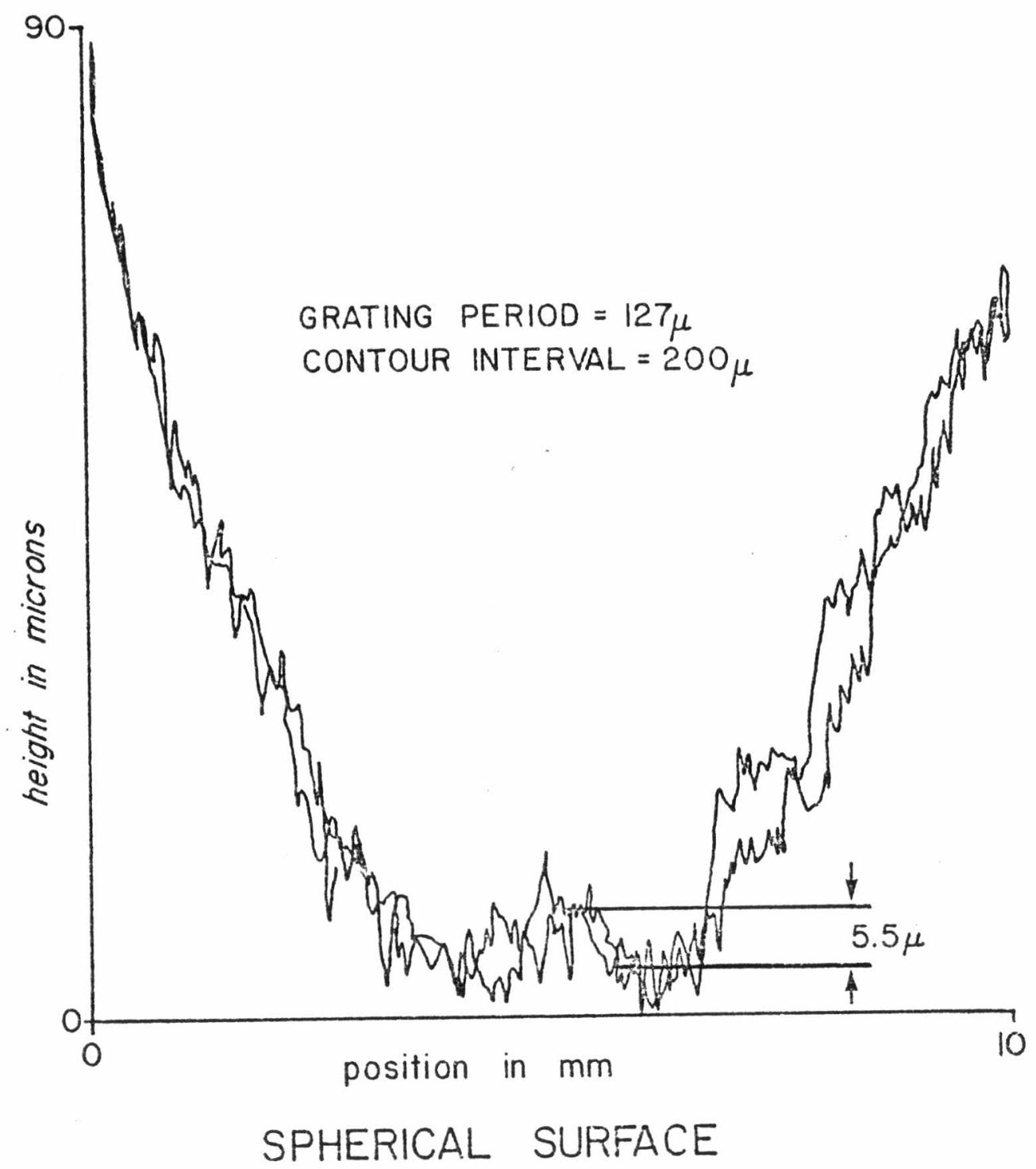

Example of Resolution in the 1-10 Micron Range

Figure 4-4 
The discussion thus far has been concerned with the repeatability of the measurements. Another parameter of equal importance is the absolute accuracy of these measurements. In order to check the absolute accuracy of the system a special test object was used. This object has a "V" shaped groove $290 \pm 10 \mu$ in depth and $9 \mathrm{~mm}$ in width. The sample was mounted as nearly parallel to the grating as possible with an estimated slope error of no more than $20 \mu$ over a $10 \mathrm{~mm}$ width. The instrument was then set up to contour one of the sloped faces of the groove from top to bottom. Two measurements were made with a $200 \mu$ contouring interval, one of the graphs is shown in Figure 4-5. The measured groove depths from the two graphs are $285 \mu$ and $300 \mu$ with an error of $20 \mu$ or $\Delta z / 10$. The magnitude of the error in this measurement is greater than what is normally observed. This is due to the fact that the pinhole size was large in relation to the fringe spacing in the image. The measured values of the groove depth both agree with the actual value within the experimental error of the instrument. 


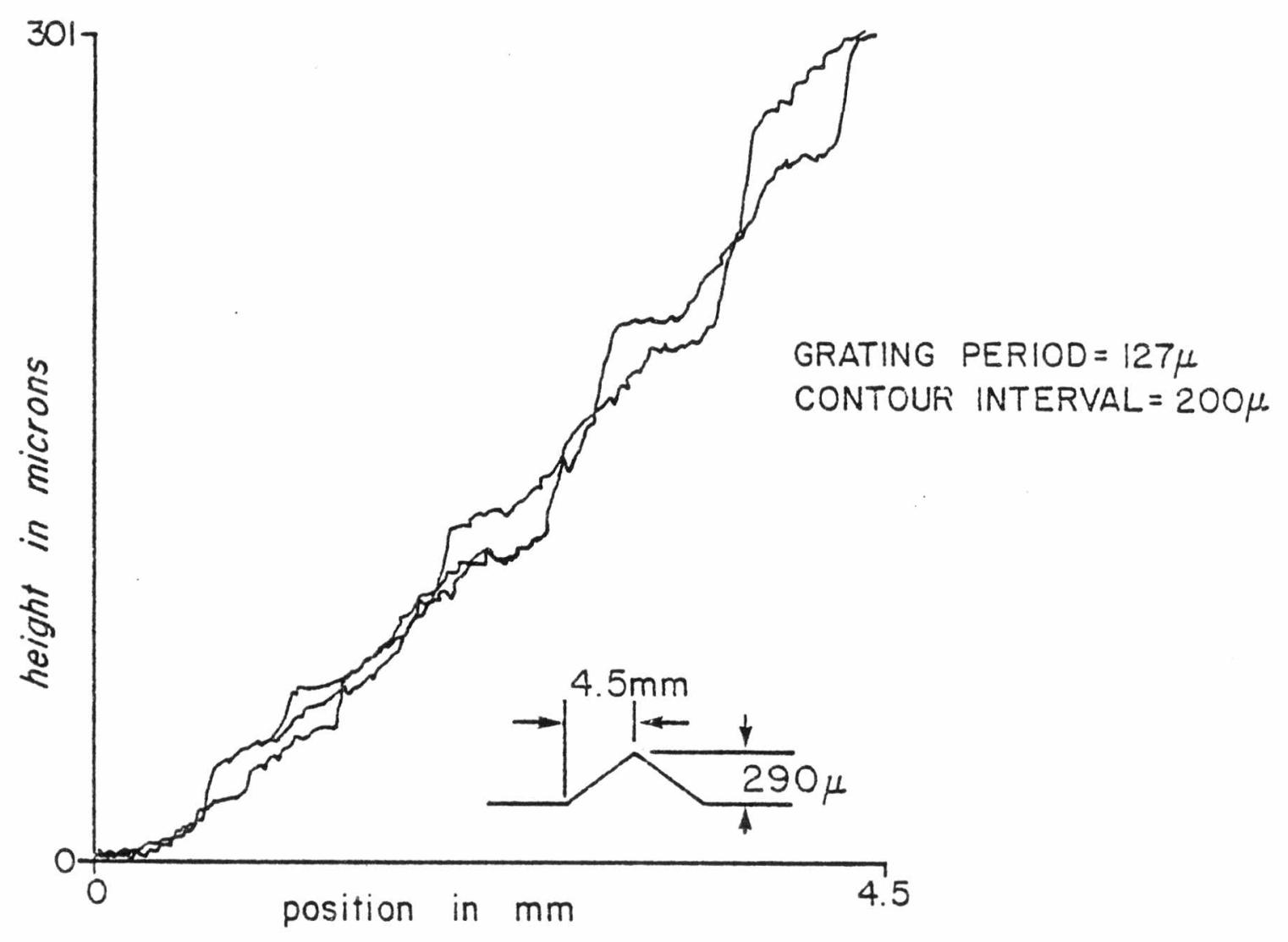

TEST OBJECT

Contour of Calibration Sample

Figure 4-5 
One of the possible applications of this instrument is to contour machine parts which unfortunately, are rarely painted white. To show that objects with metallic surfaces can be contoured two such objects were measured. The first object was Washington's face on the front of a quarter. The graph of the data is shown in Figure 4-6. Observe the retrace error at the upper right, this has a maximum error of about $29 \mu$. From a visual inspection of the surface the upper part of the curve appears to be the best representation of the surface. The second metallic object chosen for contouring is a gear cutter used in the production of Wankel Engines (Figure 4-7). Again observe the retrace error which, at two separate points is clearly caused by the jumpy operation of the motor.

Attempts were made to contour a ground glass surface but it has not yet been possible to obtain a fringe pattern with high enough contrast. The limiting factor appears to be due to light transmitted by the ground surface and then backscattered by the rear surface washing out the shadow pattern of the grating. Since the contrast of the shadow is reduced a corresponding reduction in the contrast of the moire' pattern is observed.

In its current configuration the instrument has two major limitations. The primary limitation which was mentioned previously is the slow scanning speed. An increase in the current speed by a factor of two is possible 


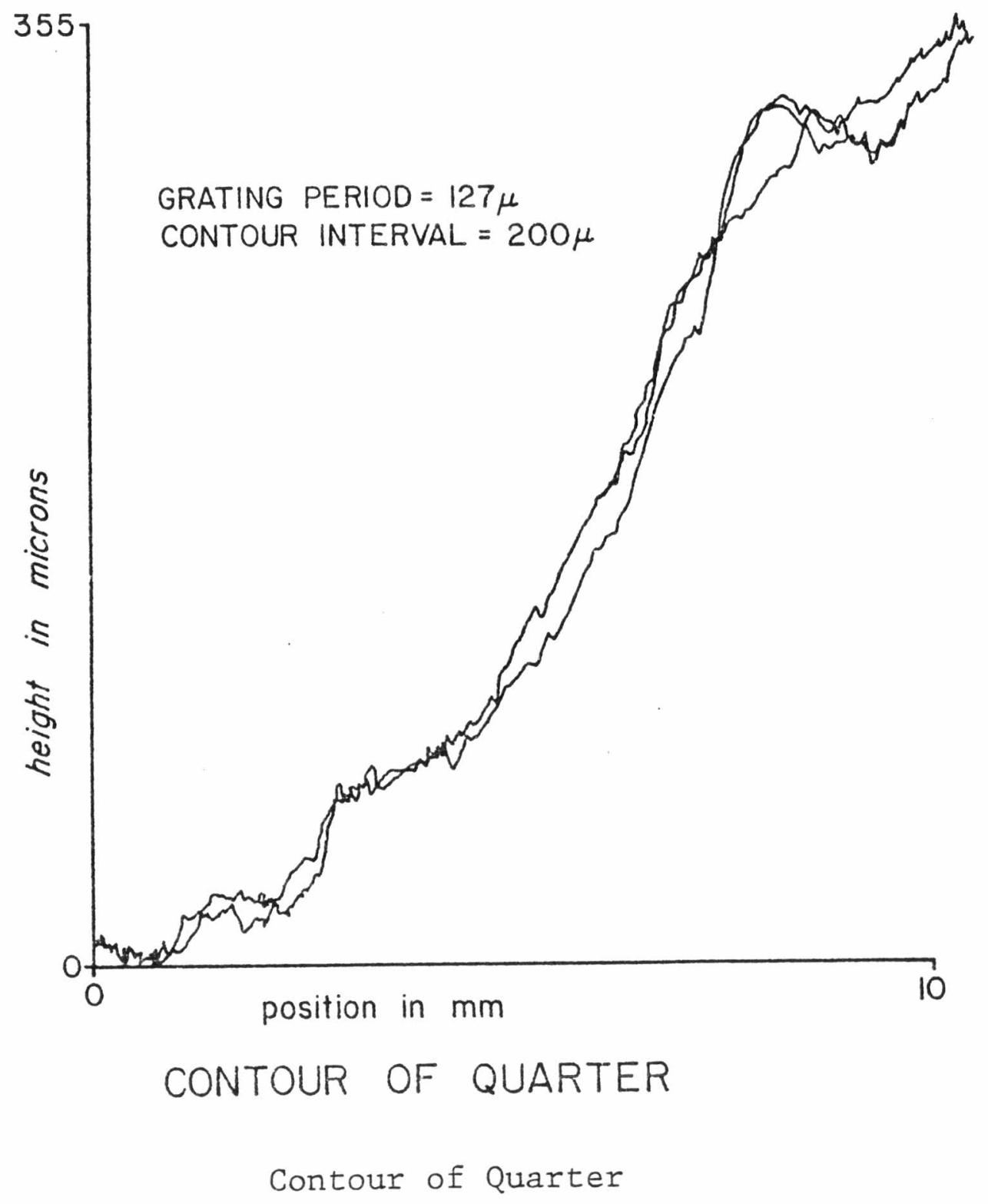

Figure 4-6 


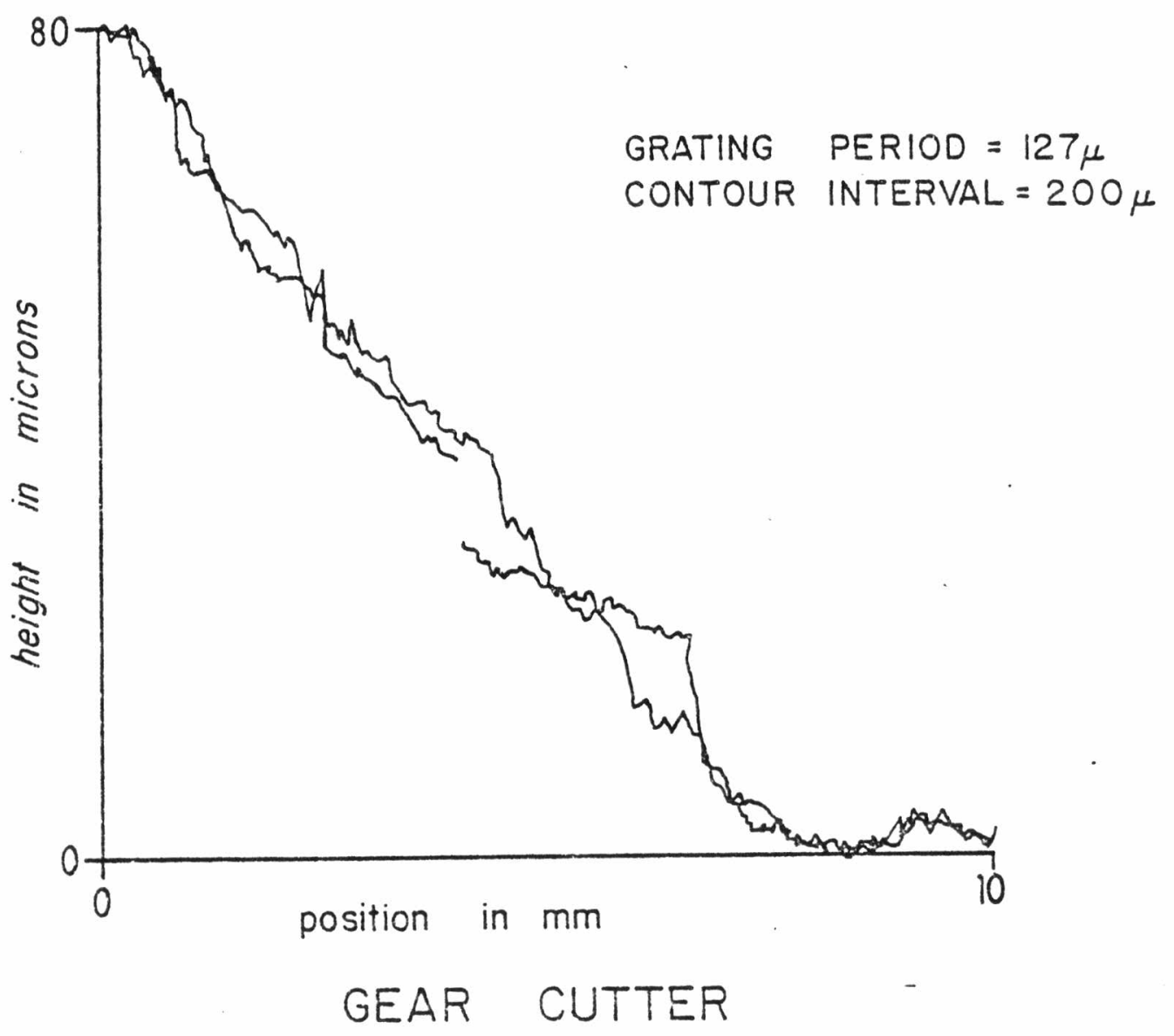

Contour of Gear Cutter

Figure 4-7 
without introducing contouring errors, any further increase is not feasible unless the motor drive is modified. The second limitation is in lateral resolution which is limited to one millimeter by the size of the pinhole. Because of the signal to noise ratio of the electronics, the required optical signal strength forces the minimum pinhole size to be no less than one millimeter.

At this point mention should be made about the type of grating best suited for moire' contouring. The two most commonly available gratings are chrome on glass and emulsion on glass. The emulsion on glass gives a much higher contrast than the chrome on glass. This results from the fact that the dark lines on the emulsion grating absorb the light which strikes them while the chrome bars scatter some light to the observation point thus reducing fringe contrast. Even though the contrast is lower with a chrome on glass grating it is still suitable for contouring. An example of a measurement made with a chrome on glass grating is shown in Figure 4-8, where the object has a white, painted surface. Notice again the retrace error due to the jumpy operation of the motor. 


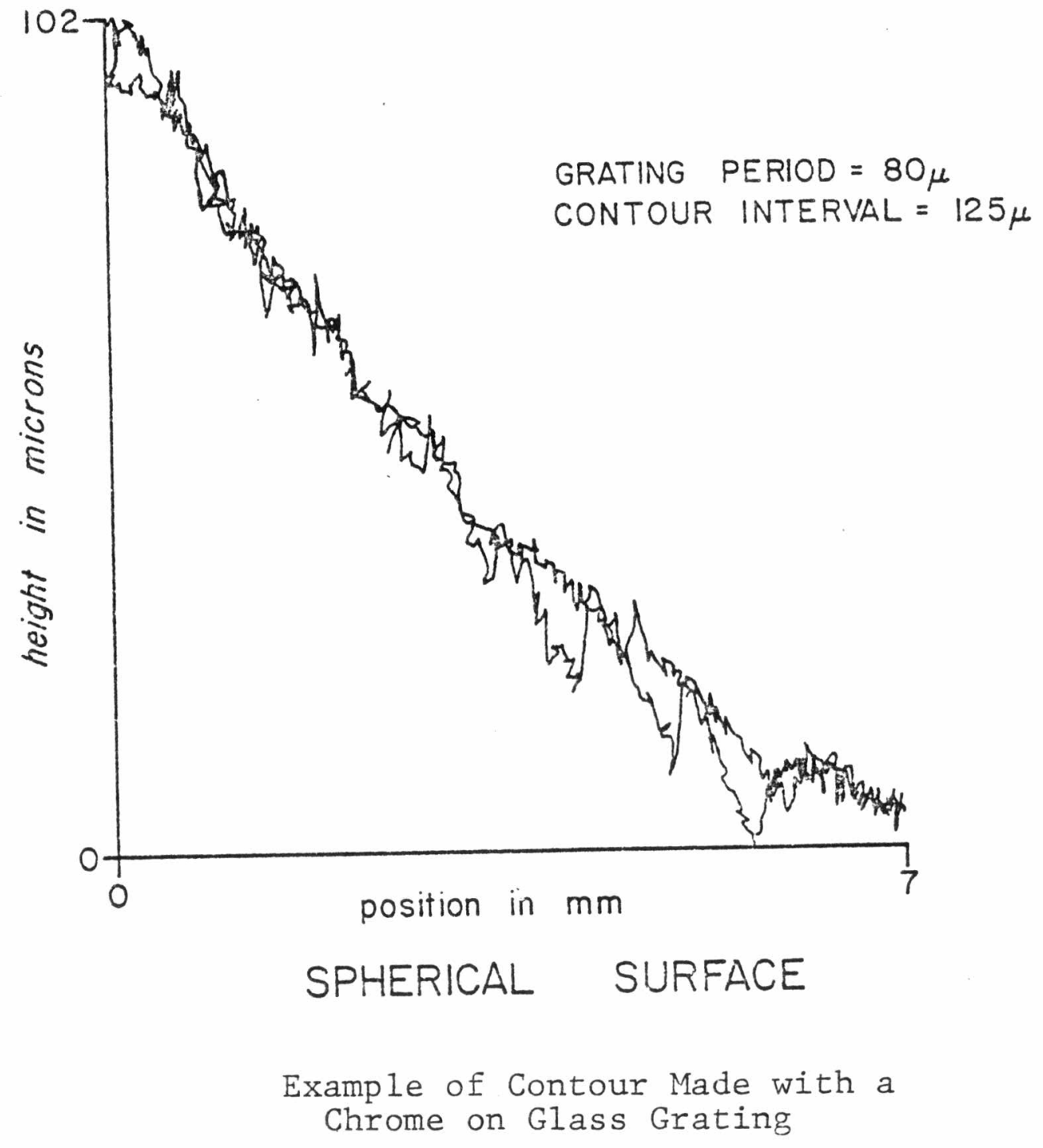

Figure 4-8 


\section{CHAPTER 5}

\section{SUMMARY}

The results presented in the previous chapter show that automated contouring of diffuse surfaces by moire' shadow techniques and phase-locked fringe detection is feasible. Further development of this instrument could result in a very useful tool for many laboratory and manufacturing applications. This chapter assesses the operation of the phase-locked moire' contouring system and makes recommendations for future work. 


\subsection{Conclusion}

One of the primary goals of this instrument is the capability of automatically contouring surfaces with a vertical resolution of one micron. The results show repeatable accuracies represented by the value of $E_{\text {typ }}$ of one-twentieth of a fringe. This implies that one micron resolution would require a grating with a period of about 12 microns producing a 20 micron contouring interval. From Eq. (29) it is found that a 12 micron grating only has a contouring depth of about 70 microns. Since contouring must be done on no less than the fourth fringe from the grating to produce useful modulation a grating of this period cannot be used. There are indications that resolution in the $\Delta z / 40$ range are possible with some modification. If this is achieved then one micron resolution is feasible, using a grating with a 25 micron period producing a 40 micron contouring interval. Such a grating would have a usable contouring depth of about seven fringes.

Measurement of ground glass surfaces was one of the objectives which prompted a desire for a system of this type. Because of the properties of ground glass such measurements have not been possible. The solution to this problem may be in finding a method for coating the surface with a very thin layer of some light scattering substance such as white paint or a fine white powder. Of course any such coating would be required to be non-destructive 
and easily removed.

The scanning rate currently used to make measurements is about fifteen seconds per line. At this speed a sixteen line two-dimensional scan would take about four minutes. This represents an increase in contouring speed by more than an order of magnitude over the photographic techniques now in use. Certain improvements in the electronics can speed this up even further.

The scanning rate is limited by two characteristics of the electronics. One problem which has already been mentioned is the stepping action of the stage drive. The second limiting factor is the time constant of the filter used to clean up the error voltage. If these problems are remedied,a scanning speed of five to ten times the current value would be possible thus reducing data acquisition time to less than one minute for a sixteen line scan. Such an improvement is desirable if the instrument is to be practical in a production environment where large numbers of objects need to be measured.

In order for this system to realize its full potential a number of modifications need to be made. The most important is the operation of the motor drive. It is imperative that the motion of the stage be smoothed out if repeatable, accurate measurements are to be made. One approach to this problem is to install a regulated speed control on the motor. This produces a constant motor 
speed which is directly proportional to the magnitude of the error voltage signal. An alternative approach is to replace the analog D.C. motor with a digital stepping motor. The motor would be driven by a train of pulses whose frequency is proportional to the magnitude of the error voltage. Such a motor would be required to move the stage in sub-micron steps in order to assure resolution in the one to ten micron range.

A second important modification is concerned with improvement of the signal-to-noise ratio in the analog signal processor. This can be done by designing a quieter amplifier section and replacing the preamplifier with a lock-in amplifier. When installing the lock-in amplifier there is the important consideration concerning chopping frequency. This must be at least ten times the value of the highest frequency of interest present in the optical signal. Such a consideration would require a chopping frequency of at least $40 \mathrm{kHz}$. With these improvements it should be possible to realize an improvement in the signal to noise ratio by one order of magnitude.

Once the stage drive and processing electronics are improved an imaging system capable of two dimensional scanning should be installed. This will require a redesign of the imaging optics such that they are capable of scanning the complete aperture with a minimal amount of distortion. An additional feature of the imaging system 
which could prove itself very useful is the capability of variable image magnification. Also a new set of optics for the illuminating beam scanning system is needed since the current arrangement does not have a large enough aperture to completely illuminate the grating.

The final step in modifying the hardware is to set up a number of status bits so the computer can discriminate between good and bad data and shut down the system if need be. These status bits should be of a diagnostic nature so that the problem can be easily remedied. There are two common occurances which disrupt contouring and are primary candidates for status bits. The most frequent error is the phase-locked loop which synchronizes the sample and hold with the resonant scanner either shifts phase or looses lock completely. The second common problem is that the object will run into the grating if a fringe of too low an order (where the order number is $z / \Delta z$ ) is used for contouring. When either of these conditions occur the computer should be notified and the stage drive disabled to prevent damage to the grating.

Currently the phase-locked moiré system is competitive with the majority of other moire' contouring methods mentioned previously. Once these improvements have been made this instrument should be capable of contouring diffuse objects with moderate speed and with resolution approaching one-fourtieth of a contour interval. With this type 
of performance phase-locked moire' will be superior to any other moiré technique now in use.

A drawback of this instrument is that the size of the object is limited. Even if the system is scaled up in size, the size of available gratings will impose a limit. This is a problem of the mechanics of the system. The electronics on the other hand are capable of analyzing any oscillating fringe pattern. For this reason future work in this area should be directed towards developing a method for producing oscillating moiré patterns on objects of any size.

If the goals put forth in this final chapter can be accomplished phase-locked moire' contouring will prove to be an extremely versatile measuring instrument for the characterization of diffuse surface profiles. 


\section{APPENDIX 1}

\section{BOARD DESCRIPTIONS}

A1 Amplifiers and filters the detector signal from the moire' pattern.

Contains automatic gain control, $4 \mathrm{kHz}$ tuned amplifier, $2 \mathrm{kHz}$ tuned amplifier and level detectors for the DC bias level and AC signal level.

Trimpots include; DC too high, DC too low, AC too high, AC too low.

A2 Generates the pulses for the sample-and-hold compensation circuits, rectifies the error voltage and drives the motor direction relay.

Contains Phase-Locked Loop, pulse generators for the sample-and-hold and compensation circuits. It also contains the rectifier and level detector for the error voltage.

Trimpots include adjustments for the phase and aperture of the sample-and-hold pulses, PhaseLocked Loop frequency, motor speed and the switching voltage for the error voltage level detector.

A3 Produces the error voltage and generates the compensation signals at $2 \mathrm{kHz}$ and $4 \mathrm{kHz}$.

Contains the sample-and-hold and related amplifiers and filters. It also has the logic and tuned amplifiers for producing the compensation signals.

Trimpots include error voltage offset and gain adjustments and four phase and amplitude adjustments for producing the desired compensation signal.

FC Detects and counts fringes from the Fabry-Perot interferometer on the object stage. 
Contains the fringe detector, counting logic, 14-bit binary counter and a Digital-to-Analog converter which generates an analog output of the stage position.

Trimpot for voltage output range of D-to-A converter.

M.D. Amplifiers and switches the rectified error voltage to drive the motor.

Contains motor direction switching relay and power amplifier.

R.D. Drives the resonant scanner and generates the synchronization signal for the Phase-Locked Loop.

Contains a driving amplifier, amplitude stabilization for the scanner and a pulse generator.

Trimpot for adjusting the amplitude of the mirror oscillation

SC Contains the rectifier for the motor power supply, power switch and motor direction switch. 
Board Location

$\begin{array}{lcc}\text { Board } & \text { Box 非 } & \text { Slot } \\ \text { A-3 } & & 1 \\ \text { A-2 } & & 2 \\ \text { A-1 } & 3 \\ \text { FC } & 4 \\ \text { DDIN } & 5 \\ \text { LED } & 6 \\ \text { GALA } & 7 \\ \text { GALB } & 8 \\ \text { DDOUT } & & 9 \\ \text { CI } & & 10\end{array}$

Box 非

Board

Slot

Galvanometer Regulator Galvanometer Driver

1

R. D.

M.D.

Regulator $(5 \mathrm{~V},+15 \mathrm{~V},-15 \mathrm{~V})$ 


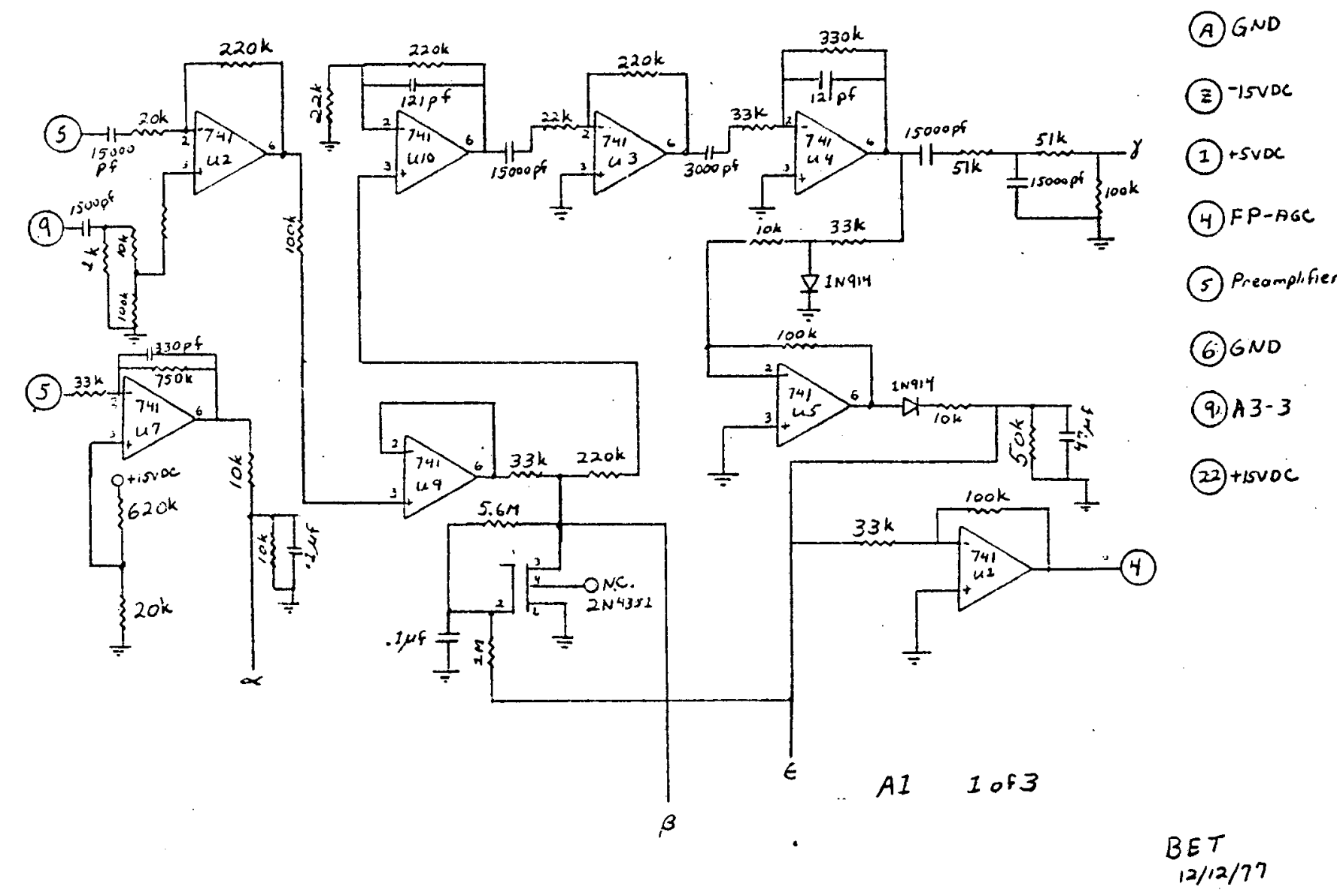




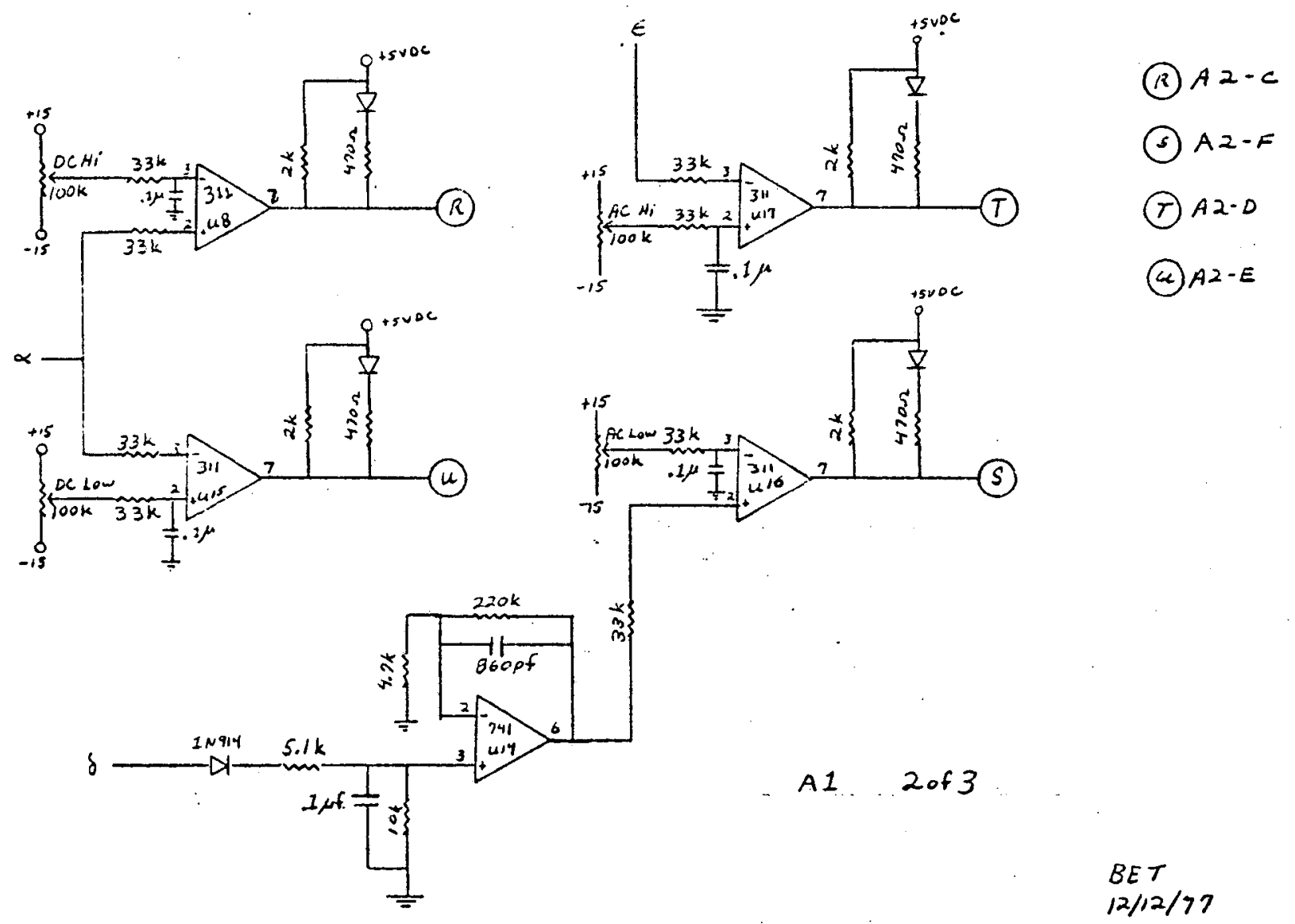




\section{$4 L / x^{\prime} / x^{\prime}$ \\ $1 \exists \theta$}

\section{$\varepsilon f^{\circ} \varepsilon \quad$ I}

$\stackrel{\text { L }}{0}$

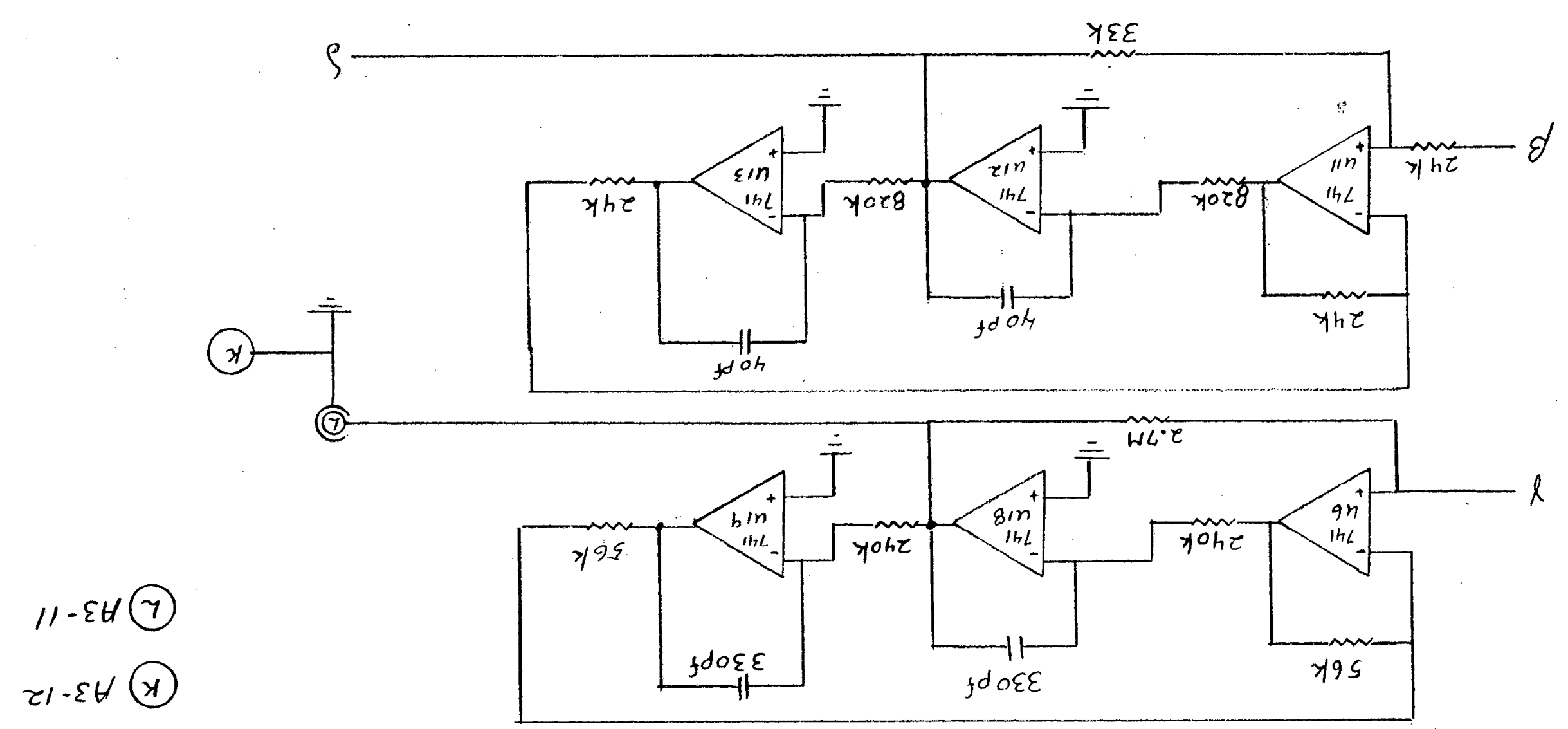




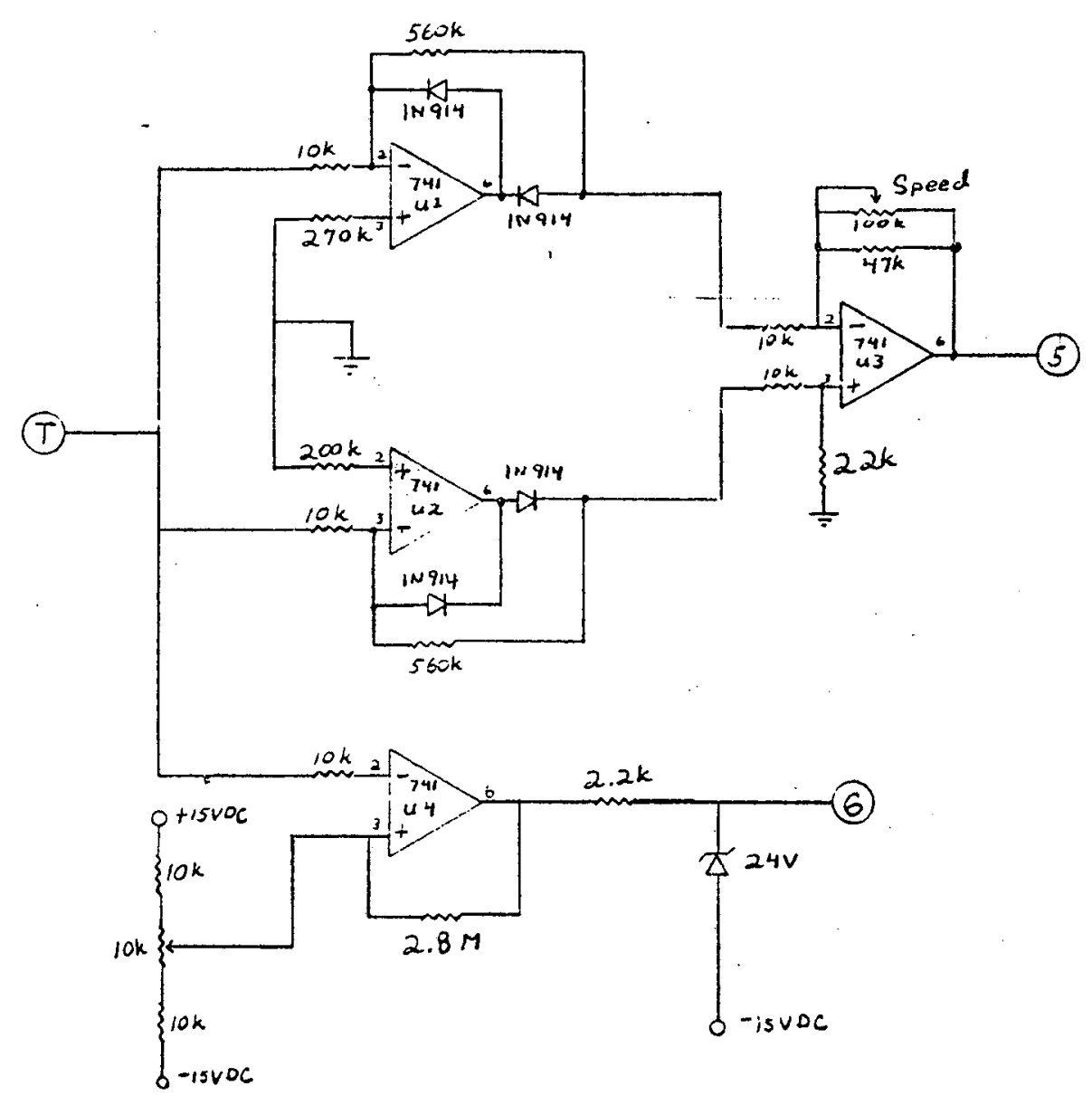

(J) $13-13$

(2) $-15 V 0 C$

(1) $+5 \vee D C$

(5) M.O.-E

(6) MO-S

(22) $+15 \vee D C$

(A) GND

A. $\quad 1$ of 2

BET $12 / 10 / 77$ 


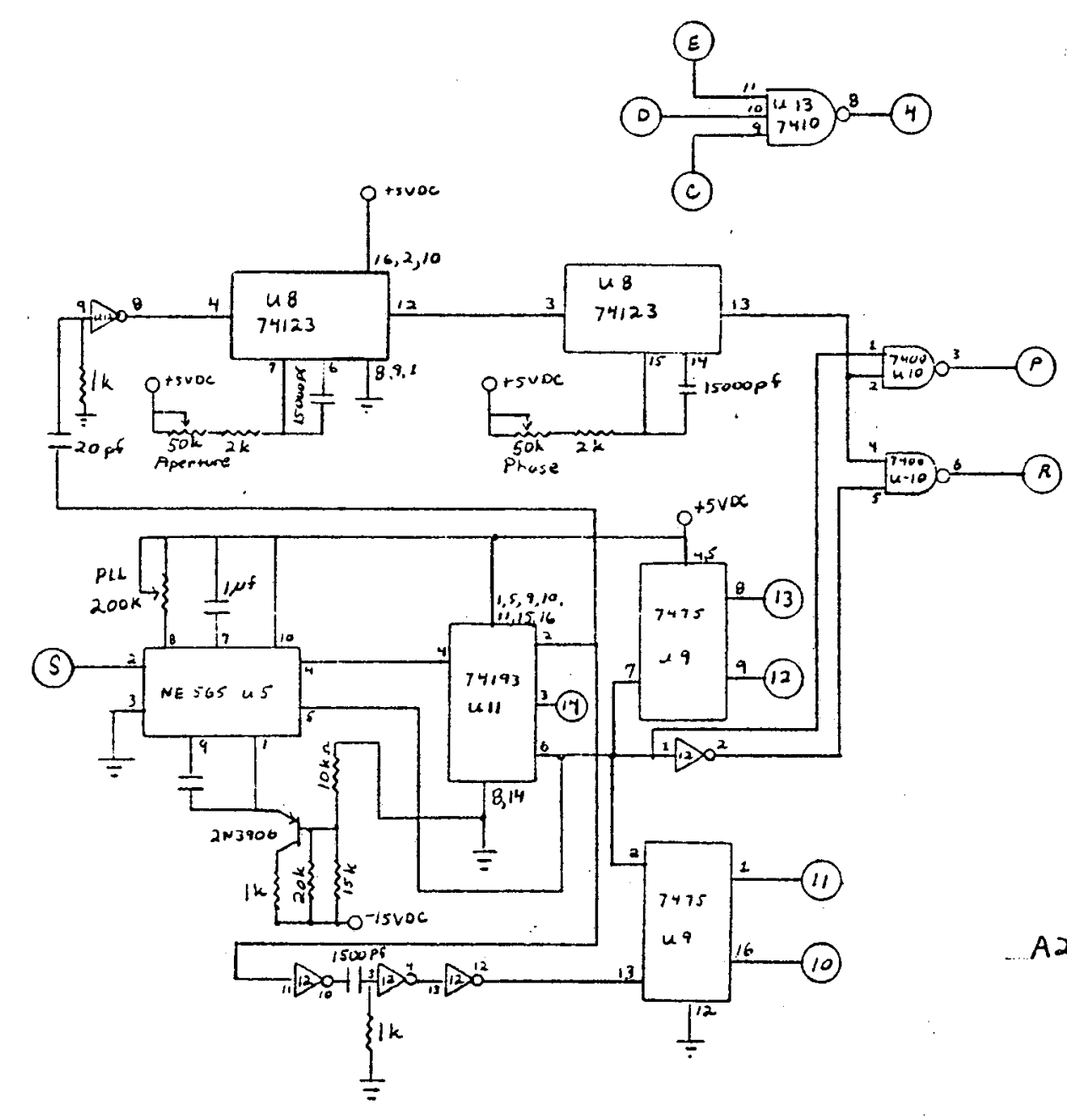

(C) $A_{1}-R$

(D) $A 1-T$

(E) $A_{2}-u$

(P) $A 1-17$

(a) $A 1-14$

(5) $R D-3$

(4) $00 \mathrm{NN}-4$

(10) $A 3-5$

(11) $A 3-6$

(12) $A 3-7$

(13) $A 3-8$

(14) $133-9$

A2 2062

BET

$12 / 10 / 77$ 


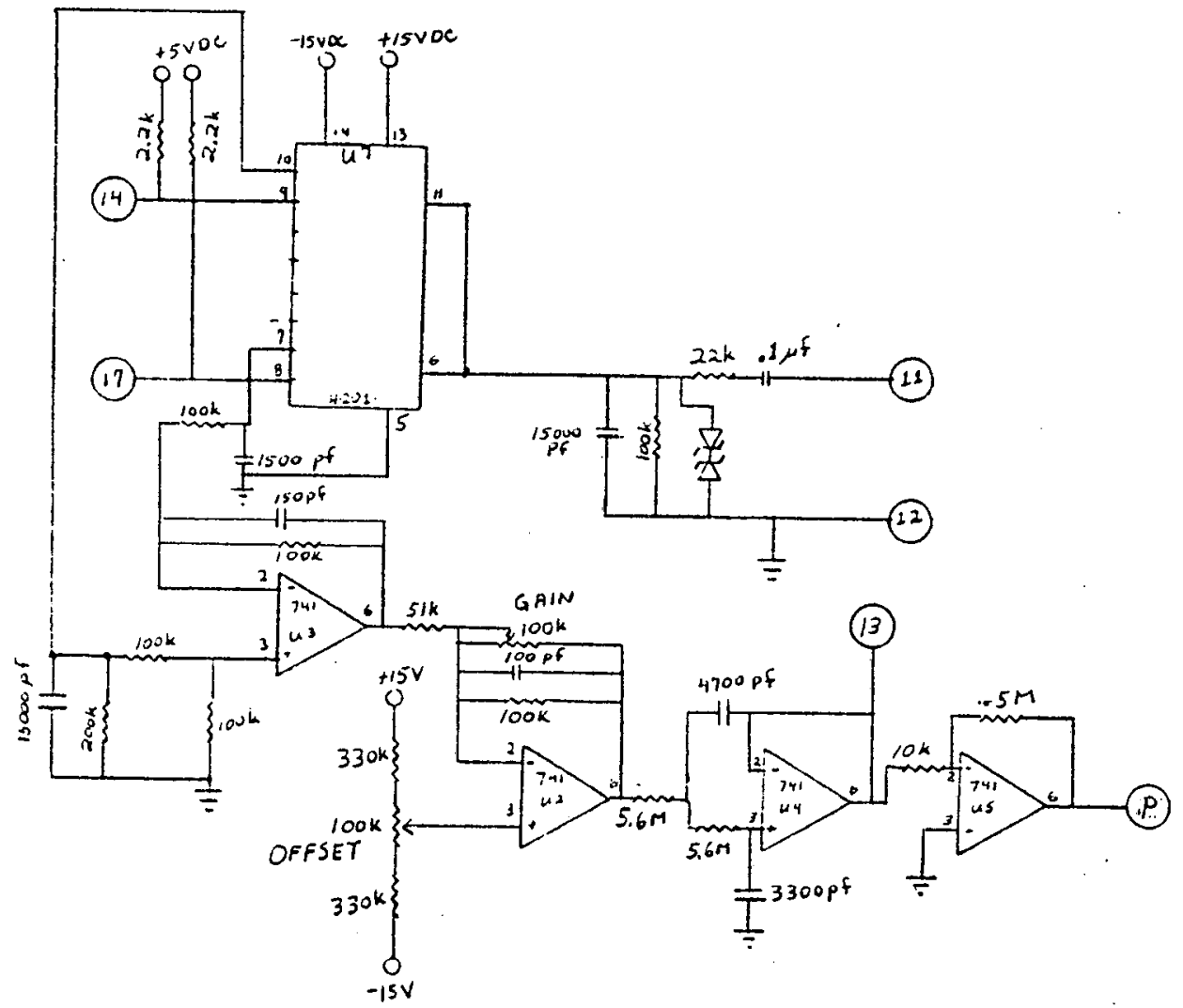
(A) GND
(2) $-15 \mathrm{VDC}$
(1) $+5 V D C$
(ii) $A 1 \cdot 2$
(12) $81-K$
(13) $R 2-T$
(14)

A-3 1 of 2 


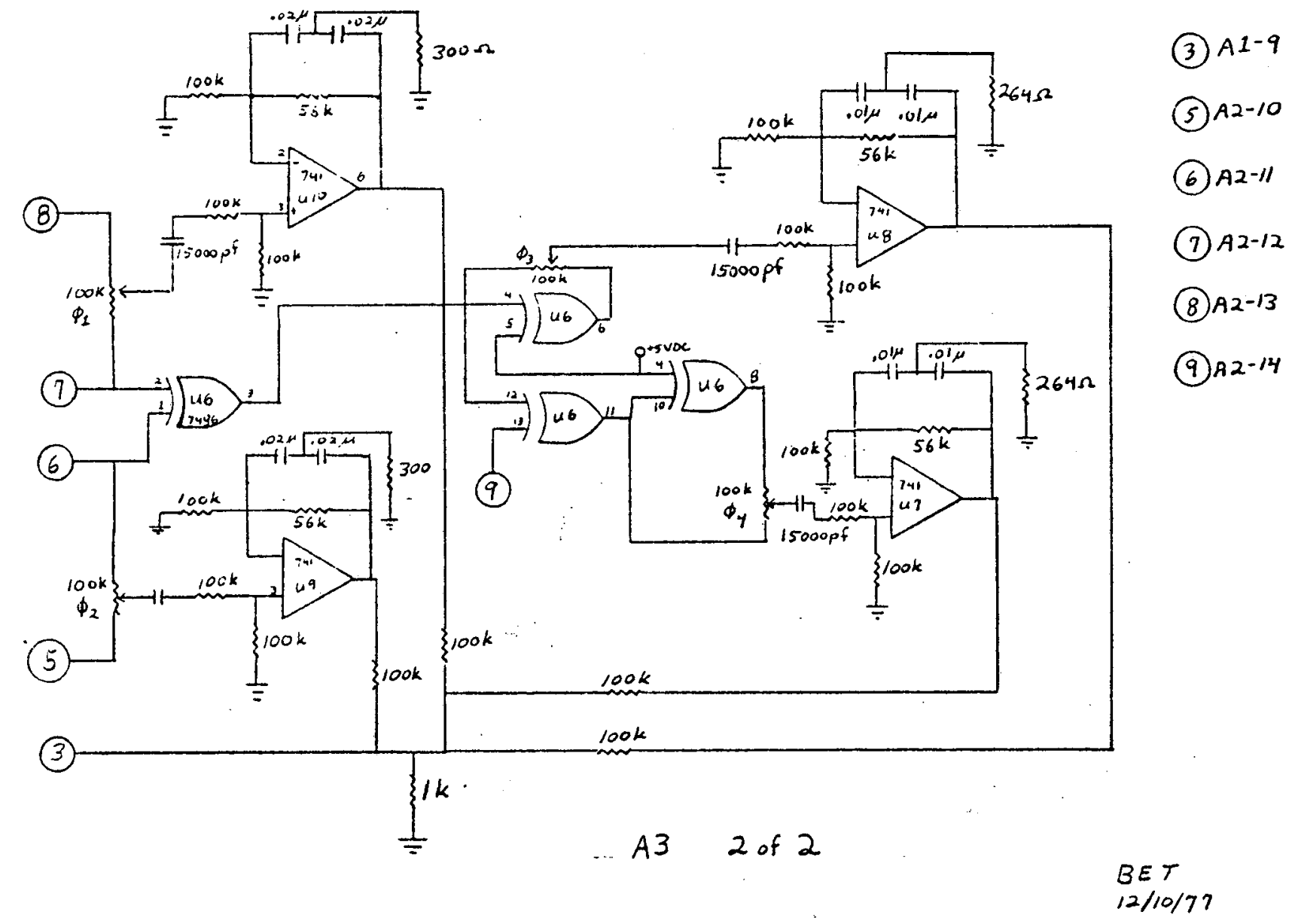

官 


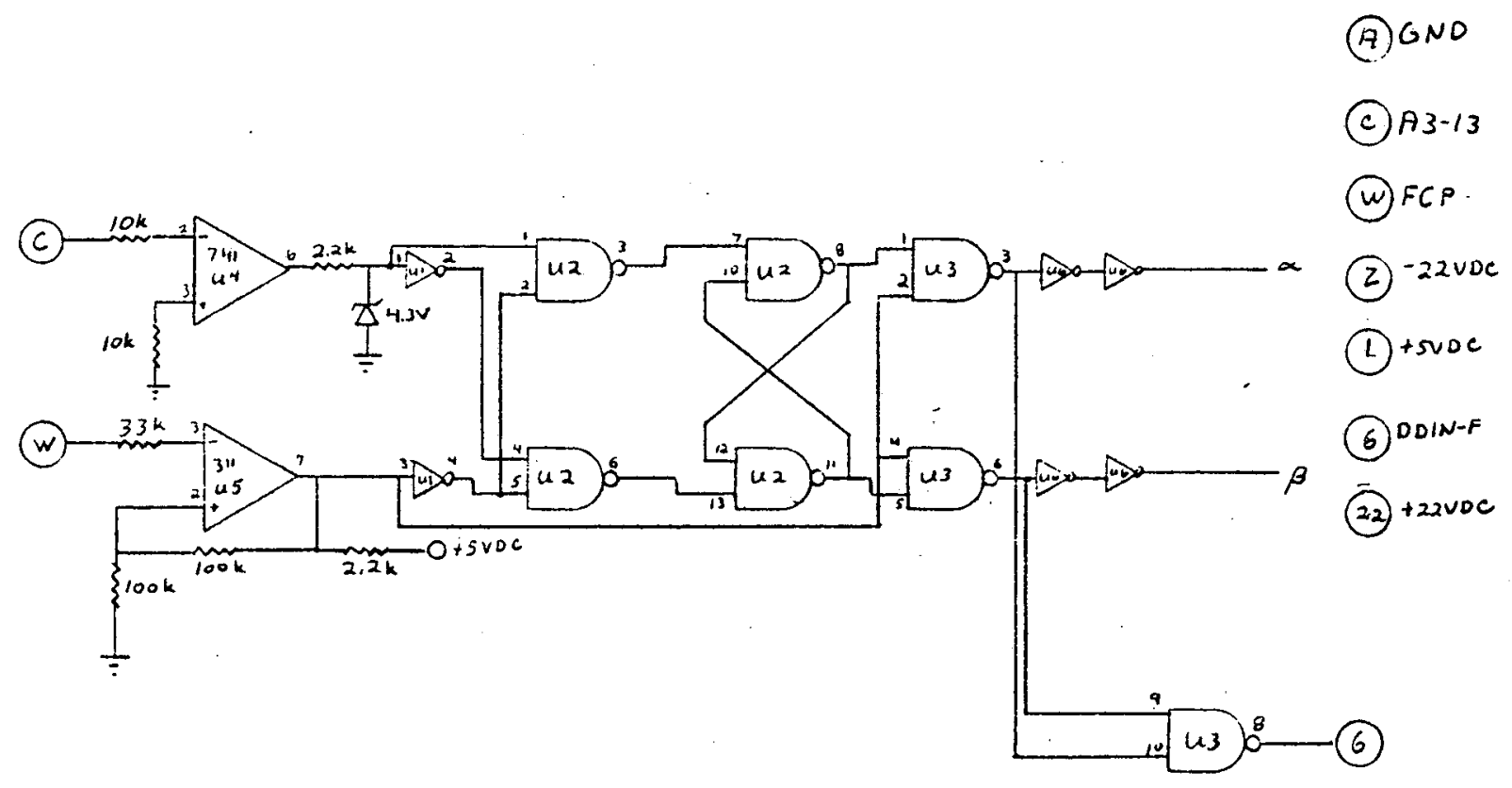

Fringe Counter 1 of 2 


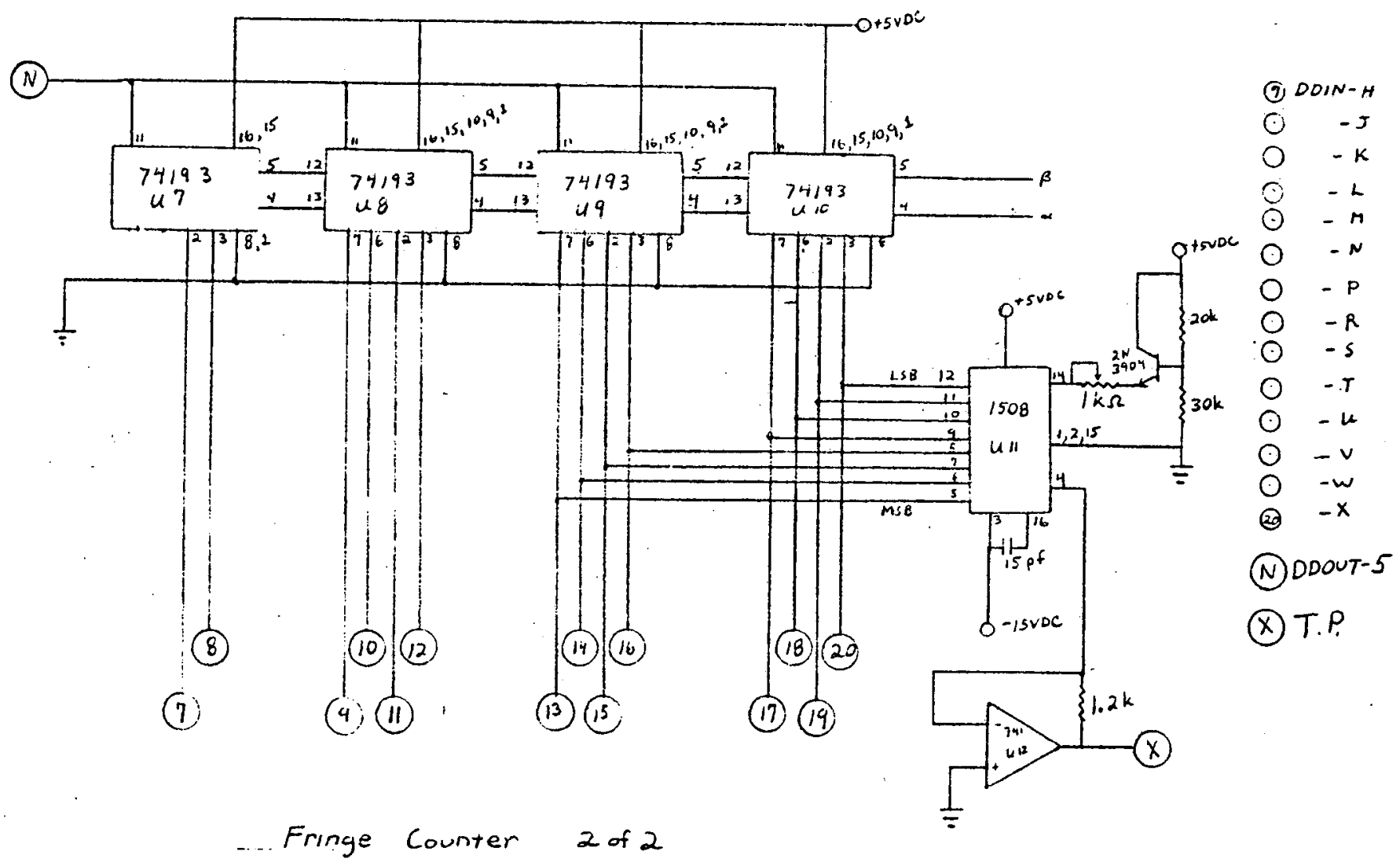




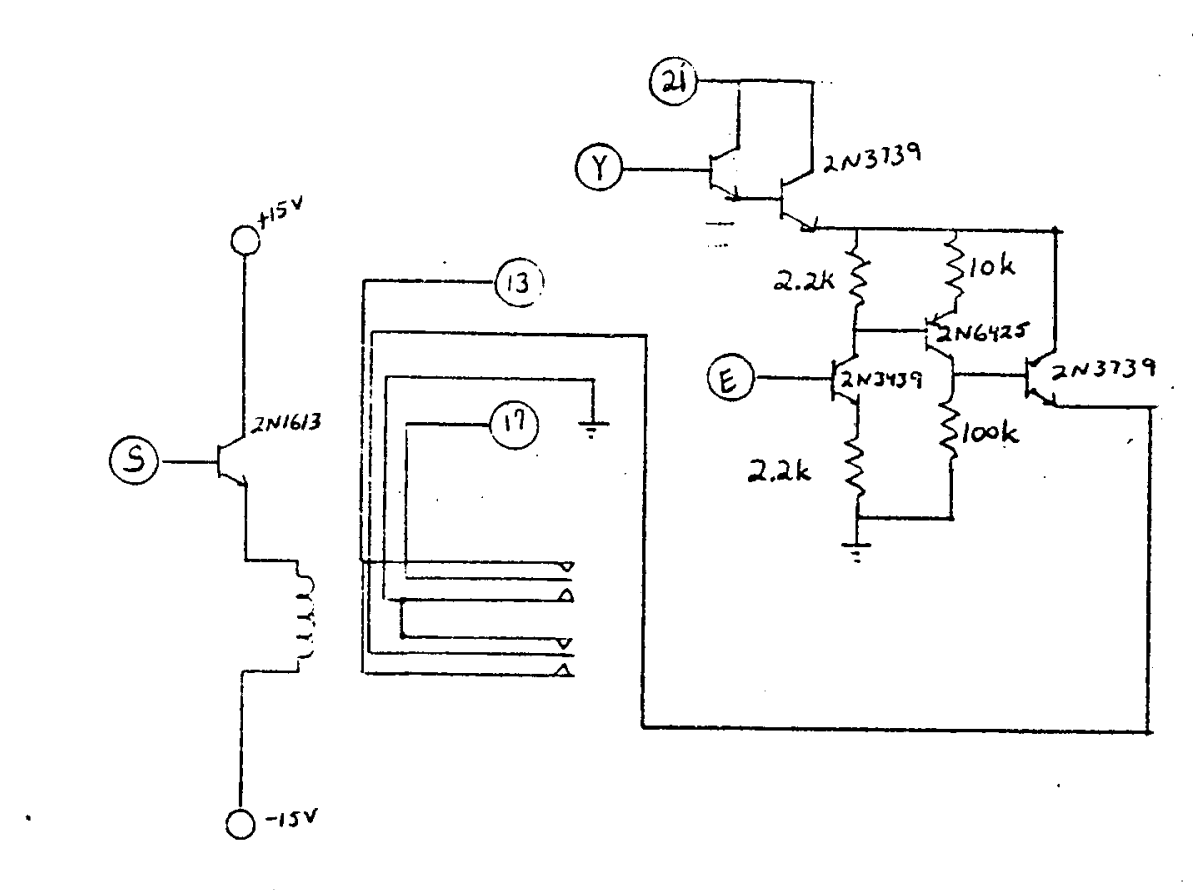
(A) Gnd
(E) $122-5$
(S) $12-6$
(Y) $100 \mathrm{~V}$ REFERENCE
(2) $-15 \mathrm{VDC}$
(13) $s c-3$
(10) $\mathrm{Sc}-5$
(2) $100 \mathrm{~V}$ P.S.
(23) $+15 \mathrm{VOC}$

\section{Motor Driver}

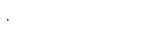




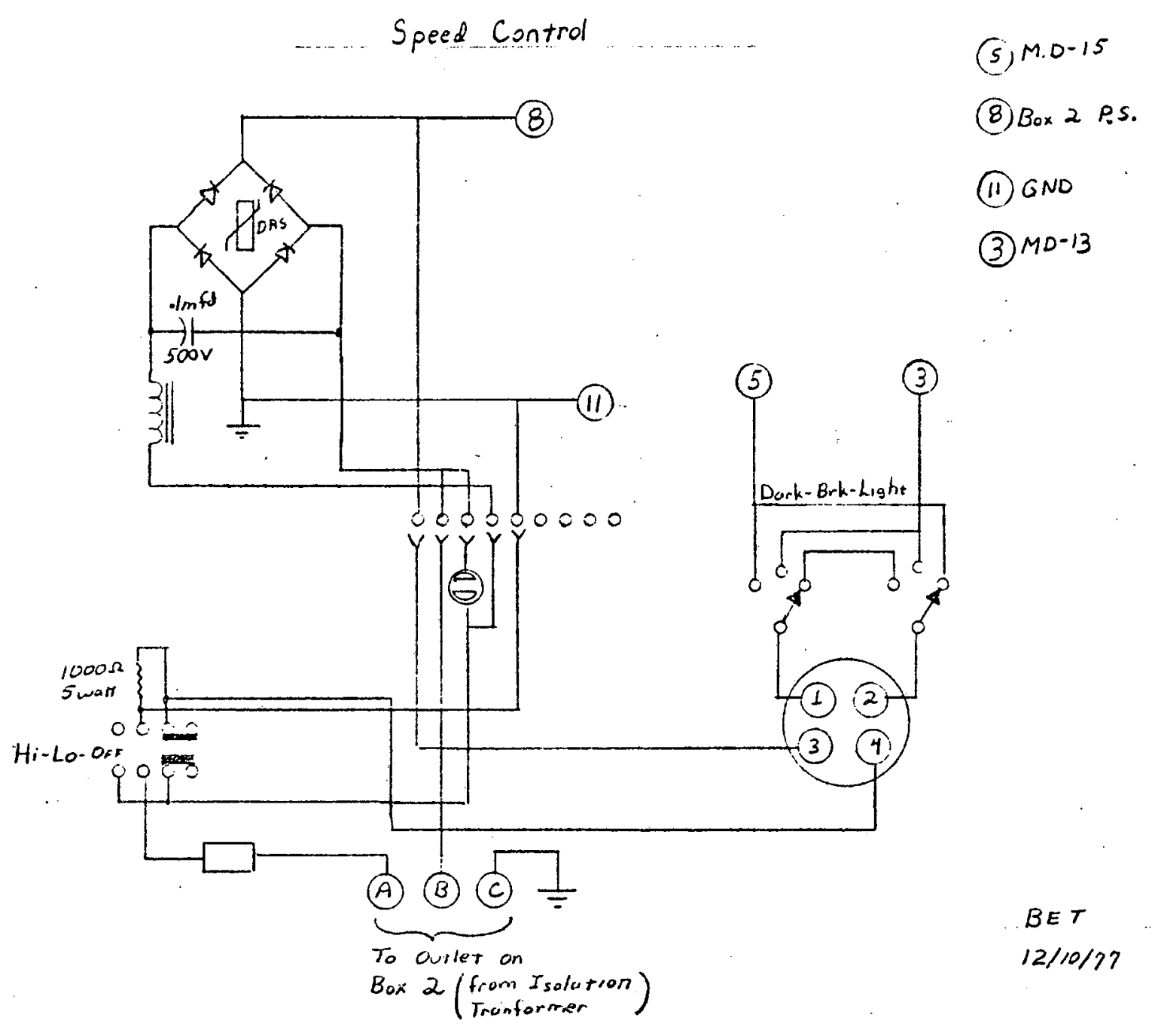




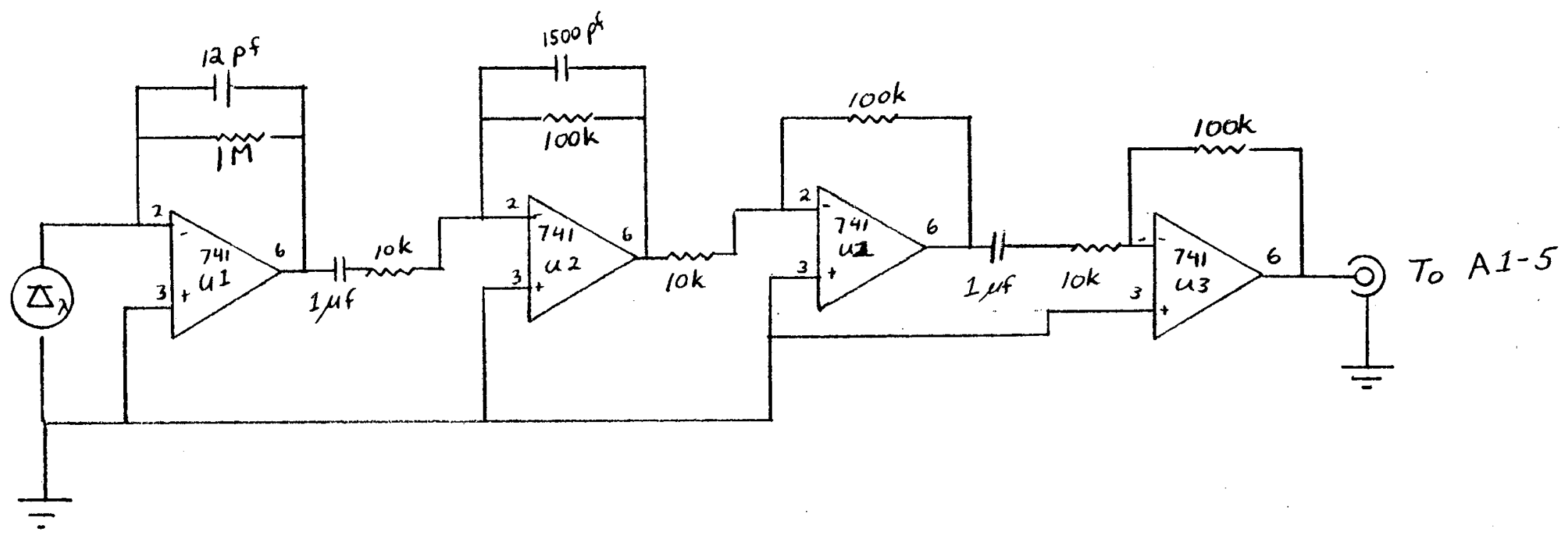

- Moire Detector Preamplifier 


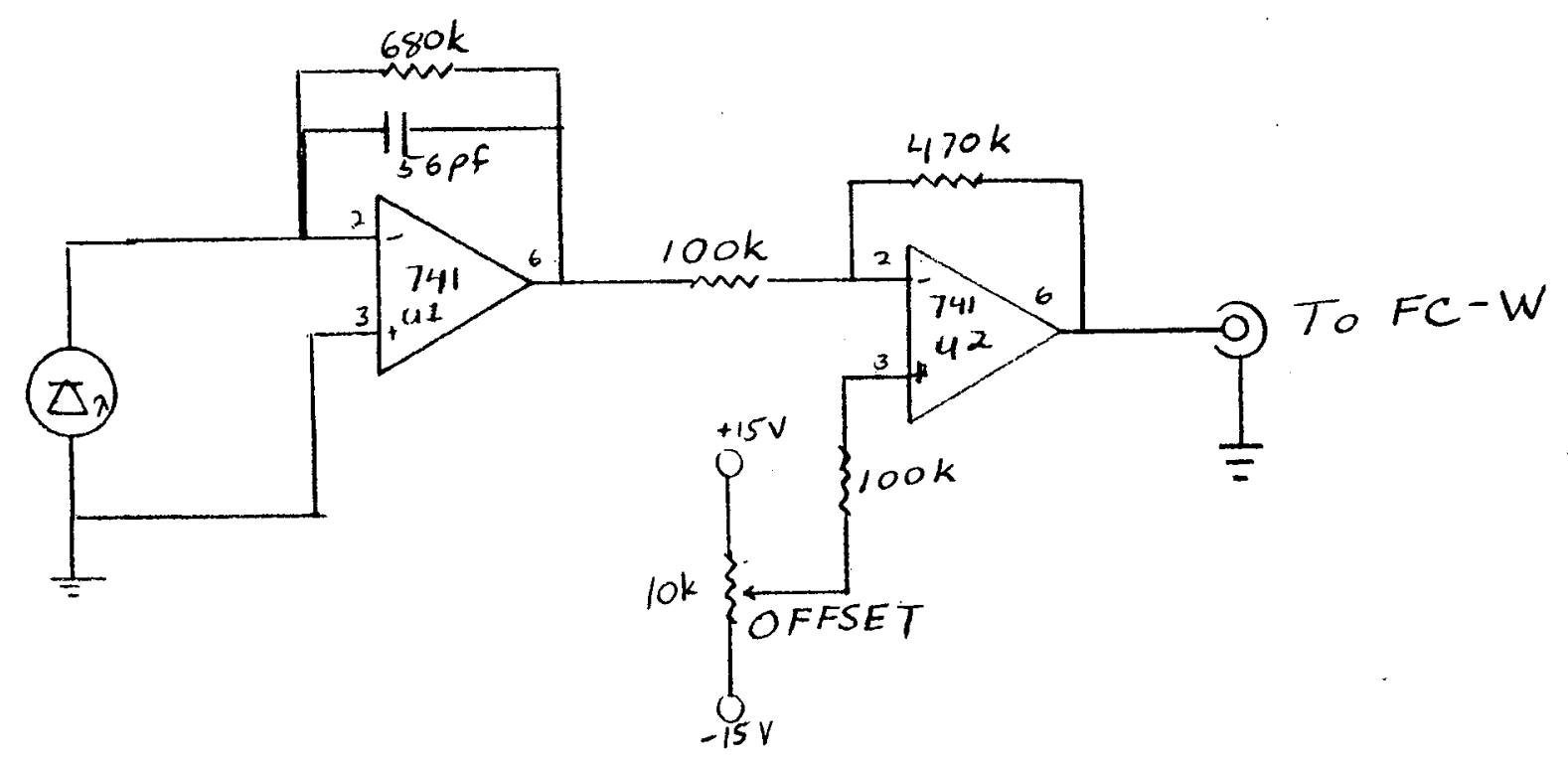

卢

Fringe Counter Preamplifier

BET

$12 / 10 / 77$ 


$$
\begin{gathered}
\text { Interconnection } \\
\text { AQ } 116
\end{gathered}
$$

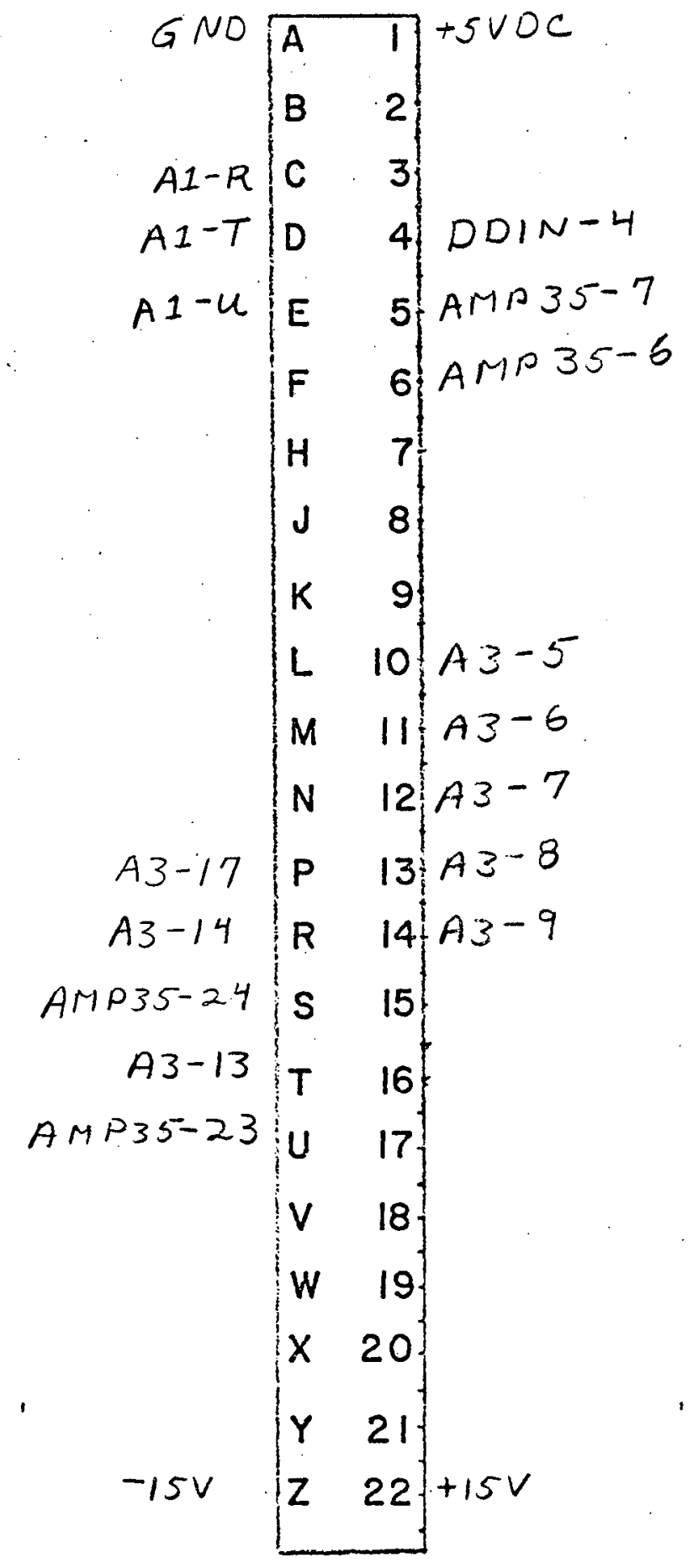




$$
\begin{gathered}
\text { Interconnection } \\
\text { AB }
\end{gathered}
$$

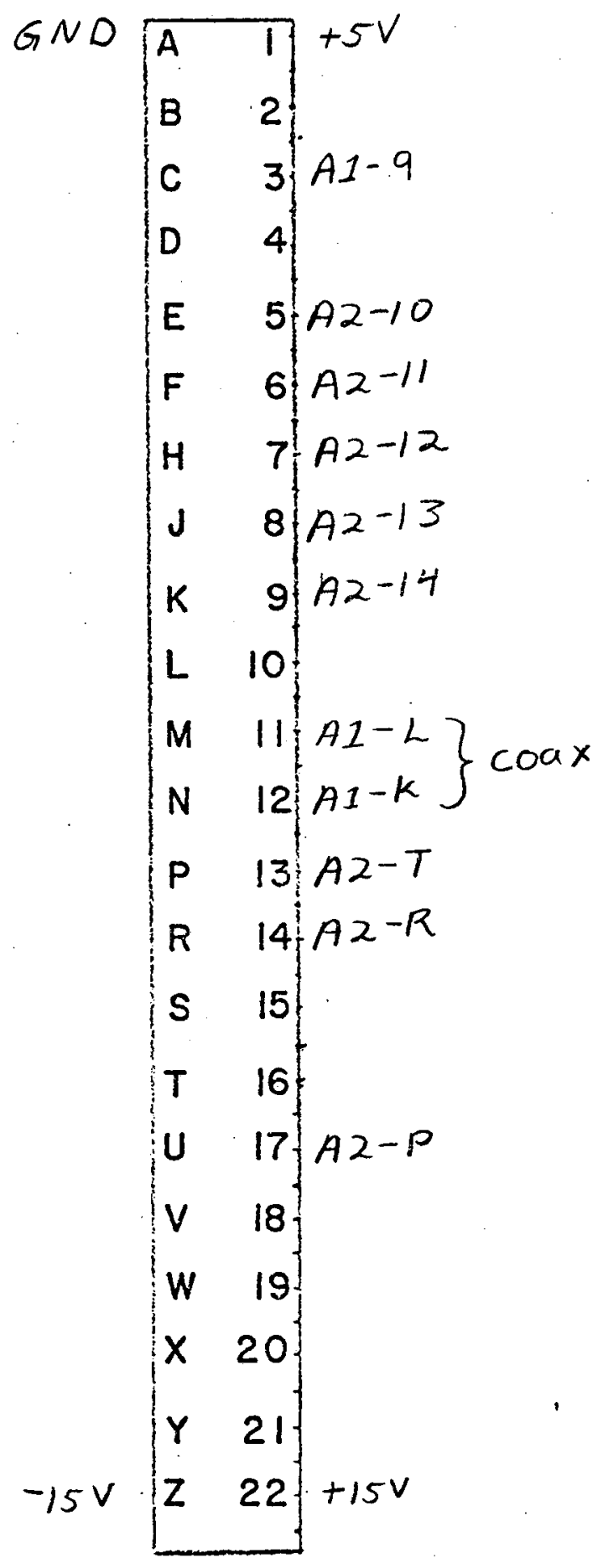


Interconnection

AI

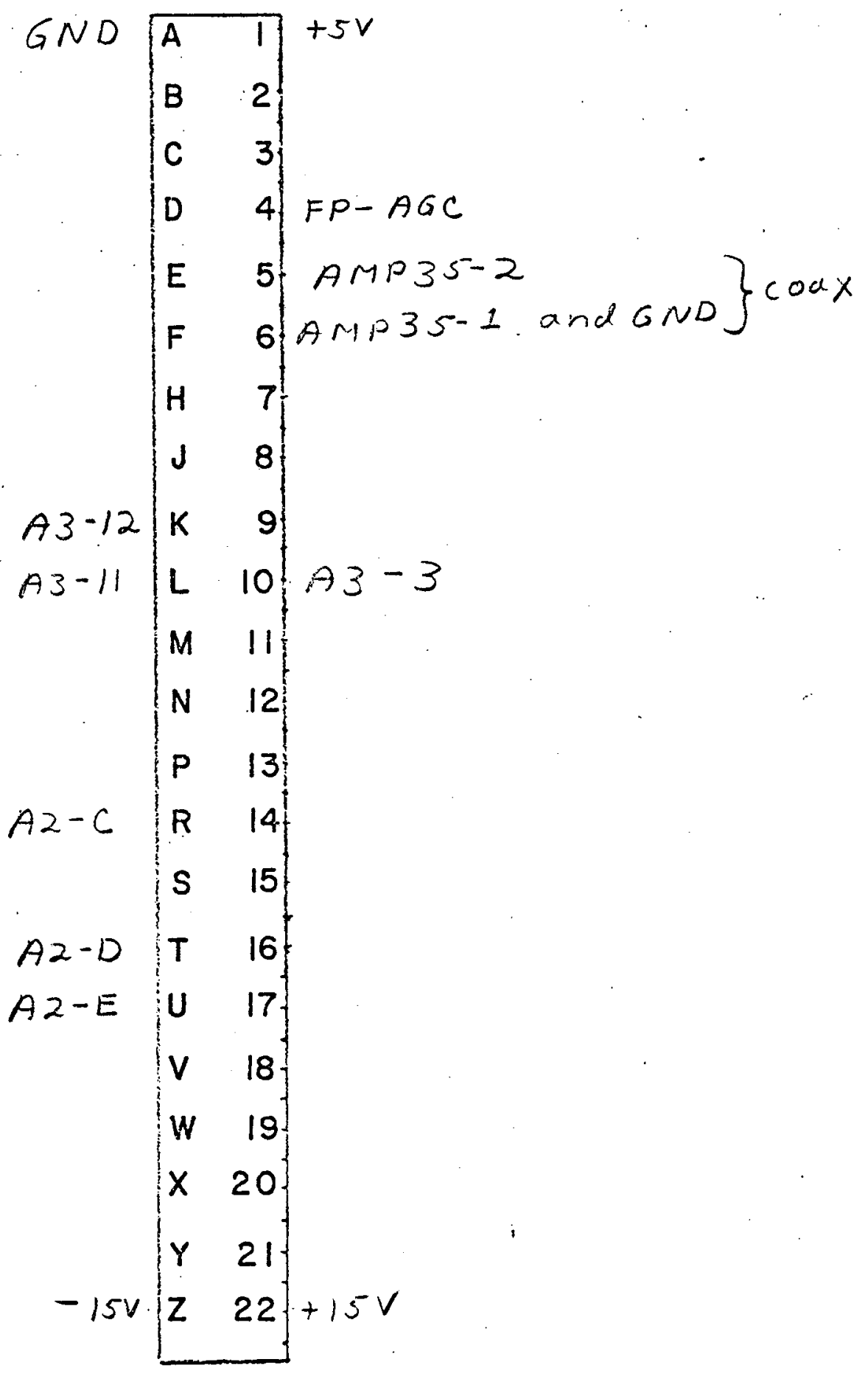


119

$$
\begin{aligned}
& \text { PLIM To Box } 2 \text { Cable } \\
& \text { PLIM END } \\
& \text { AMPHENOL } \\
& \text { 36.PIN }
\end{aligned}
$$

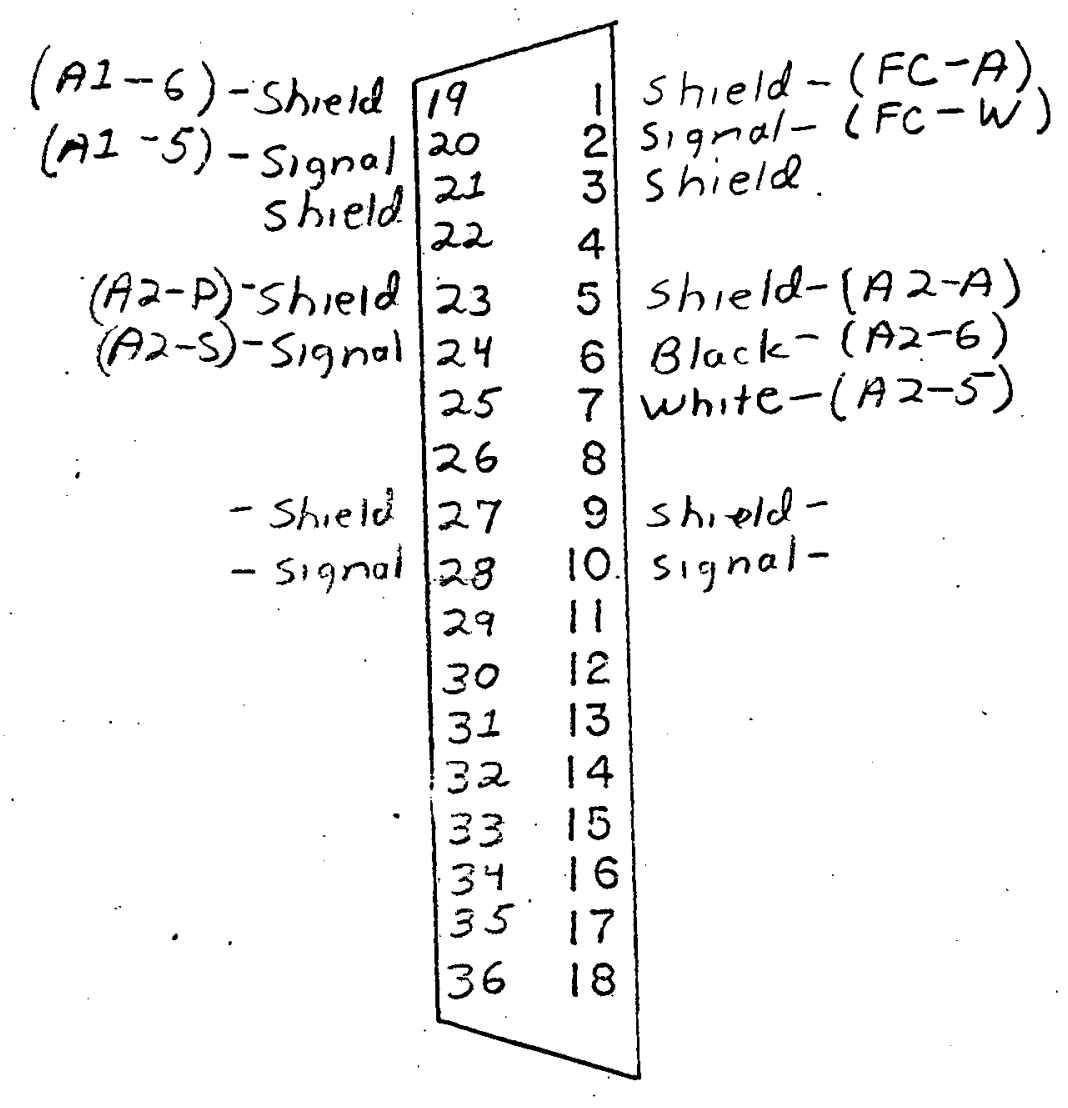


120

PLIM to Box 2 Cable

Box 2 end

AMPHENOL

36 PIN

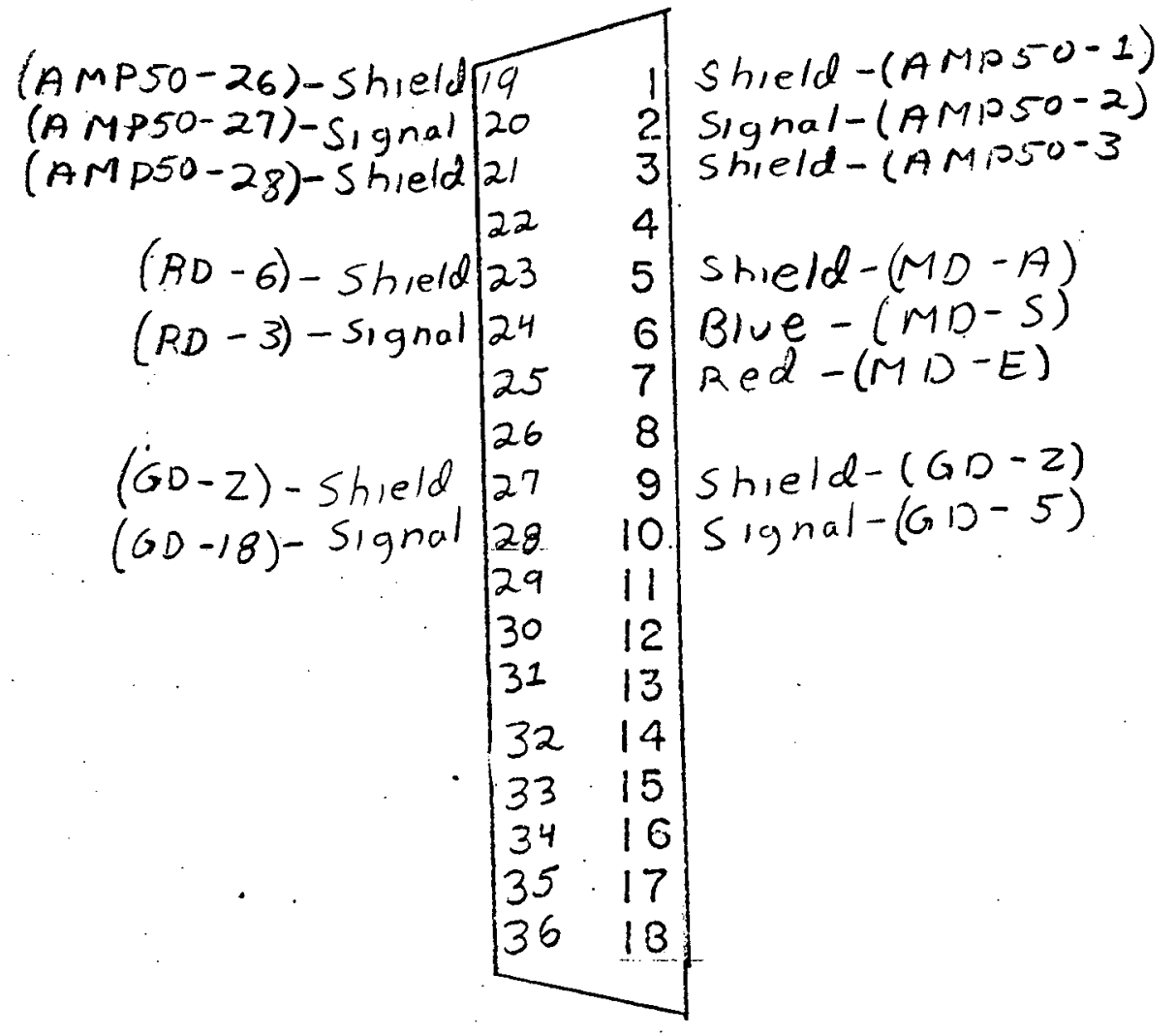


Female

AMPHENOL

50 PIN

$$
57-30500
$$

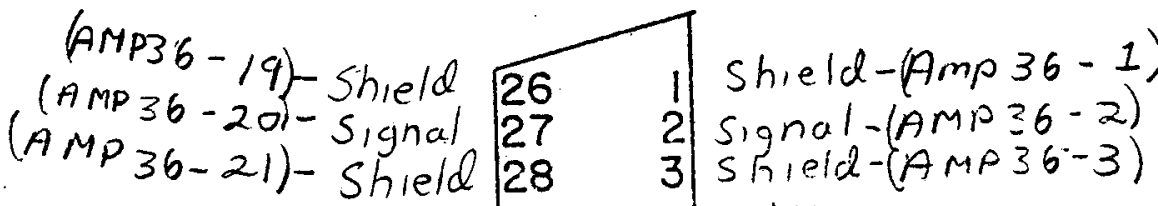

$$
\begin{aligned}
& \begin{array}{rr}
29-4 & -15 V \\
30-5 & -15 V
\end{array} \\
& M D-A \mid \begin{array}{ll|l}
31 & 6 & \\
32 & 7 & S D-6
\end{array} \\
& M D-13 \mid 33 \quad 3 \quad S D-10 \\
& M D-16 \quad 34 \quad 9 \quad 5 D-7 \\
& M D-1435 \text { 10. SD-1 } \\
& M D-1736 \quad 11 \\
& M D-A\left|\begin{array}{ll}
37 & 12 \\
38 & 13
\end{array}\right| \\
& \text { MO-H } 39 \quad 14 \\
& M D-J \\
& M D-5 \mid \begin{array}{ll}
41 & 16 \\
42 & 17
\end{array} \\
& 43 \quad 18 \\
& 44 \quad 19
\end{aligned}
$$

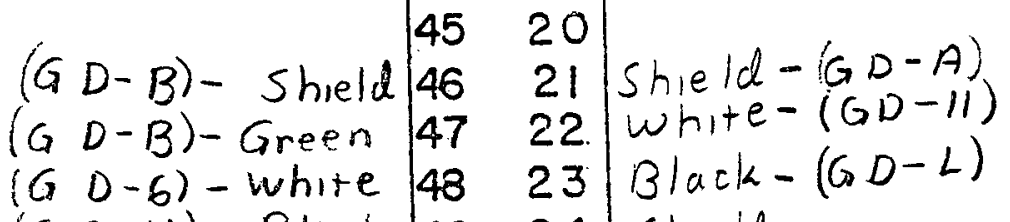

$$
\begin{aligned}
& (G D-H) \text {-Black } 4924 \text { Shield_ } \\
& (G D-4) \text {-Red } 50 \cdot 25
\end{aligned}
$$


122

Connector For Lines to Equipment

Male

AMPHENOL

50 PIN

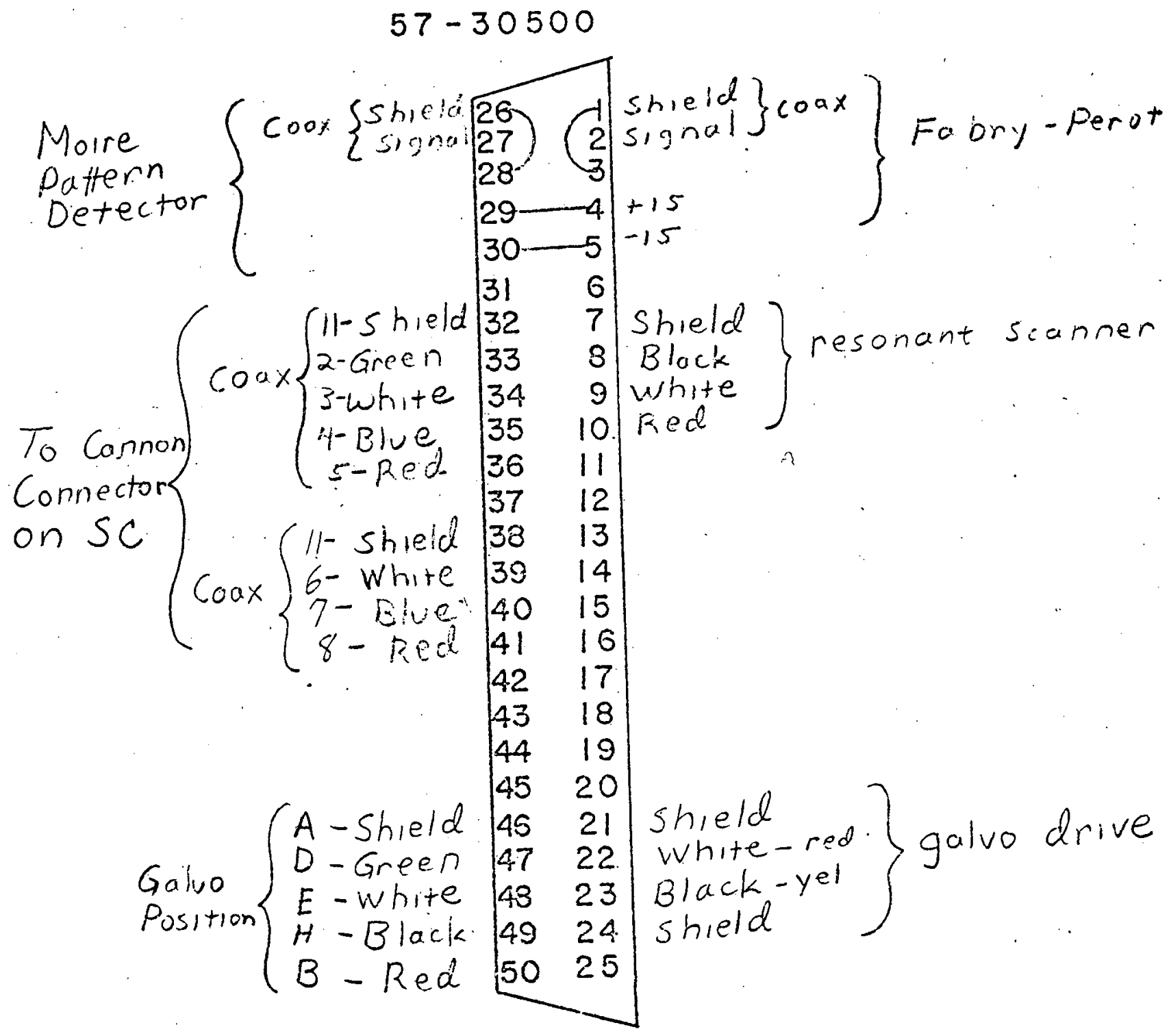




\section{APPENDIX 2}

COMPUTER OPERATING INSTRUCTIONS

The software which controls the PLIM and analyzes the data has been written for a Data General Computer which is equipped with a 5602 unit. The data acquisition program, MOIRE1, requires $32 \mathrm{~K}$ of core in Fortran IV. Due to the complexity and the size of the programs a series of overlays and swaps were necessary to minimize the amount of core necessary. The graphing and contour plotting routines are swapped with the main programs. The data is passed from one program to the other through the unlabeled common which is the same in both programs. The two programs are compiled and loaded independent of one another and the graphing or plotting SV file is called by the main program. The overlay structure consists of three areas: 1) the main program, MOIRE1, and three small programs which are used by many of the programs 2) six programs which are called by the main program 3) a number of programs which are called by the six programs in the first overlay segment. The program MOIRE1 has some extensive output when a diagnostic print code is used. For this reason, there are two output devices: the teletype or crt 
which is used for prompts and the line printer which is used for long write statements. The device codes are set in MOIRE1. To aid in tracing errors which may arise, the subroutines in which the write statements occur are printed in the left margins. This program is loaded using the command line THIMK.

MOIRE1 Prints out "A.C. MOIRE"

Prompts for the print code. There are four print codes.

$$
\begin{aligned}
& 0=\text { no output (uses default values) } \\
& 1=\text { partial output } \\
& 2=\text { partial output } \\
& 3=\text { diagnostic output }
\end{aligned}
$$

START The values of the galvanometer scaling factors and laser wavelength are set in this subroutine.

MOIREI Prompts with the option to bypass the multiple $\mathrm{x}$ scan for the microstructure measurement. 1 is the bypass code.

TSCAN Prompts with the option to bypass the noise trace. 1 is the bypass code. This subroutine should be bypassed. 
FOUR If the multiple $\mathrm{x}$ scan has not been bypassed, prompts with "NPTS=." The number of data points should be entered as a three digit number. ( 300 is the default number.)

PHASE1 The data is then taken. For output codes greater than zero, the MKI value is printed out. At this point, checks are made on the multiplex bit and data bit. A check is made to see if there is sufficient light to make a measurement.

STOR2

Prompts with "TYPE 1 TO STORE RAW DATA." If

1 is entered, a filename will be requested.

The data is then stored on the computer disk under the chosen filename and can be re-analyzed using the program ACMITT. There is also an option to print the data at this point.

For diagnostic output, 3 , the bit values and the $\mathrm{x}, \mathrm{y}$ and phase values are printed out.

Each data point is checked to verify that the $\mathrm{x}, \mathrm{y}$ and phase data are not out of bounds. If it is found that the point is not within the desired range, the message " $\mathrm{X}, \mathrm{Y}$ OR PHASE OUT OF BOUNDS" is printed out and the point is eliminated. 
For diagnostic output, 3 , prints out the bit configurations and $\mathrm{x}, \mathrm{y}$, and phase data for each point.

Prints "OVER HALF OF THE DATA POINTS HAVE BEEN ELIMINATED" if insufficient data points remain. If this error message is returned, fitted values will probably be incorrect. SCALE Prompts with the option to print the scaled data. 1 to print the $\mathrm{x}, \mathrm{y}$ and phase value with the corresponding point number.

FOUR Prints "ERROR IN PHASE MEASUREMENTS" if an error occured in the subroutine PHASE1.

FIT With diagnostic print code, prints the matrix values and the coefficients

DMATEQ Prints "MATRIX DETERMINANT IS ZERO". If the determinant is too small.

FIT Prints the seven coefficients of $A_{1}+A_{2} x+$ $A_{3} y+A_{4} x^{2}+A_{5} y^{2}+A_{6} x y+A_{7}\left(x^{2}+y^{2}\right)^{2}$ Prints the fitted values with and without an asphere fit, including the xtilt, ytilt, power, cylinder, asphere, maximum and minimum $\mathrm{x}$ values, $\mathrm{x}$ and $\mathrm{y}$ shifts, phase maximum and minimum.

Prompts with "TYPE 1 TO REMOVE TILT AND POWER" 
FOUR Prints out peak to peak and rms values

Prompts with "TYPE 1 to PLOT OUTPUT". There

is a delay before the computer responds

since the programs are swapped in.

Program returns to PHASE $I$ and takes two more sets of data.

YSCAN Prompts with "TYPE 1 to BYPASS YSCAN". This subroutine should be bypassed.

MOIRE1 A number of options can be listed out

0 to end output

1 to print data: The data printed includes the identification number, the date of measurement, the nominal radius of curvature, the actual radius of curvature, the power error, the departure from a sphere, the rms and peak to peak roughness, the asphericity and the cylinder 2 to measure another sample on the block: Prompts with "SET MANUAL STAGE" and continues with the microstructure measurements

3 to store data on disk: This option is not included in the software

4 to measure a new sample block: Prompts with "Insert Lens".

5 to list the options 
6 to remeasure the wavefront: Continues with the YSCAN and XYSCAN. 
COMMON BLOCKS

COMMON/A/

IRAY (3)

JRAY (1)

Used in XRAY calls

COMMON / B /

LAFILE

LBFIIE

LCFILE

(10)

(10)

buffer

filename

buffer

COMMON/C/

KTTYO

KTTYI

$\mathrm{KBI}$

$\mathrm{KB} 2$

$\mathrm{KB} 3$

KB4

KOUT

KDUM

KLPT

output device input device

board to 5602 a/d to computer board to 5602 PLIM to computer board to 5602 computer to PLIM not used print code variable dummy variable auxiliary output device (line printer)

COMMON/D/

XID (20)

XNA (5)

identification numbers

COMMON/E/

I7

IST

I8

$\mathrm{KZ}$

$\mathrm{KY}$

$\mathrm{KX}$

channel for overlay used in overlay not used

COMMON/F/

XFIR

YFIR

YX (64)

PPQ (64)

NLINE

a/d coordinates of first data pt (YSCAN)

a/d coordinates of first data pt (YSCAN)

$y$ data (YSCAN)

phase data (YSCAN)

total number of points (YSCAN)

COMMON

$X(500)$

$\mathrm{Y}(500)$

$\mathrm{PH}(500)$

NPTS

PII

IQ (10)

$\mathbf{x}$ data

y data

phase data

number of data points

scale factor for contour plotting unused array 
Fi:

THFE THITHE

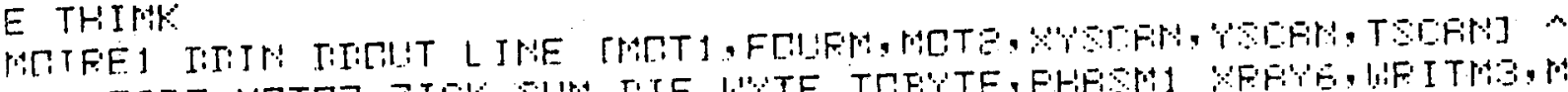

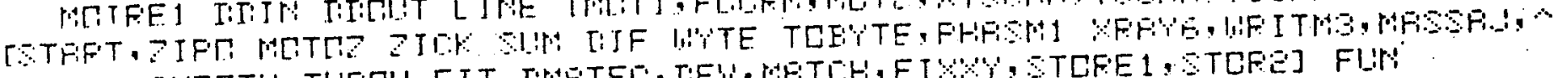

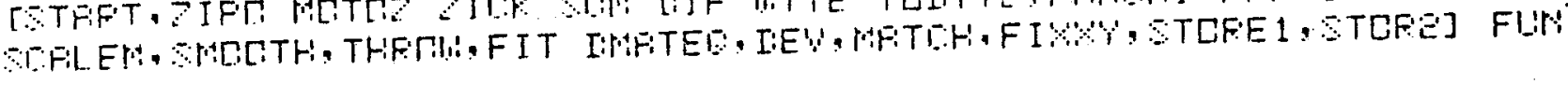

F:

EXTEFNAL LELFUFI, CAUSS, POWEFS

EXTEFNAL TAYLCIF, ULFH, UANHEEL

EXTEENAL, NEVES,FYPN, SANCHEZ

EXTEENAL HAFIY, ISAFC, CIFCOEI, KINCS, LAME, MUOFE

EXTEFNFL AEEE, EUFN, COFNU, TOVE, FAEFY

CUMMON/A' I FAY (L), JEAY(1)

COMNON /E' LAFILES $1 U)$, LEFILE, LCFILE (IU)

COMMON/C' KTTYO, KTTYI,KEI, KEC, KE3, KE4, KOUT, KLUM

COMMON/E, XIL(2U), XNACS)

COMMON $X(5 U 1), Y(5 U U), P H(5 U U), N P T S, F I I, I Q(1 U)$

COMMON/E/I7, IST, I\&, KZ, KY, KX

CONMCN/F, XFIF, YFIF, YX(64), FFG( 64$)$, NLINE

DIWENSION $\because D(5), E E(7)$

DUUELE FFECISION P(7), DET

$K T T Y I=11$

KTTYU $=10$

K.DISI $=2$

$K P 1=20 K$

$K E C=2 \perp K$

KE $3=22 \mathrm{CKK}$

C SET CHANNEL FOF DISK RFITING IN STOREI

KE $4=8$

$M D 1=1 K$

$M[2=2 K$

$M \Gamma 3=3 \mathrm{~K}$

$M \Gamma 4=7777 \mathrm{~K}$

$M C 5=40000 \mathrm{~K}$

$M D 6=7 \mathrm{~K}$

$M[7=11 \mathrm{~K}$

$M E 12=10 \mathrm{~K}$

$F E R: 2=\$ 3110$.

FEF: $\angle=11 . *$ PER:L

PEF $4=P E P$ P

C TEF- IS PEPIOI OF SCAN IN MSEC

$P E E_{1}=2500$.

I $7=7$

$N T I M=0$

$I S T=U$

IFITE(KTTYO, 13૯2)

1362 FUFMATC $* * * *$ A.C. MOIFE *****,11)

VIPITE(KTTYO, 1 US S)

IUES FORMATC" ENTER PFINT COLE, $\quad U=$ NONE, $1=$ SOME, $2=A L L ", Z)$ FEF D(KTTYI, 1 U\&6) KOUT

IUEG FOFMAT(II)

$K E F=1$

C TEMFURAPY EYPASS

$I G=1$

GO TO 533

GEITE (KTTYO, ! Uย्? ) 
1087 FOEMATC" TYFE I TO PYFASS Z KOTICN “;Z

FEAE(KTTYJ, 11186$)$ IC

533 CALL ONOFN(17, "VUIBE!.OL",IEFES)

IFCIEFES-1: $13,348,13$

13 WFITECKTTYO, 10S4) IEFPS,KEP

1054 FORNATS" EFFOP IN LOADING OVEFLAY, IEF=", I 4, " KEF=", I 4) GO TO 15

348 CALL OULOL(I7, HAFDY, IST, IEFPS)

C MOVES STAGE TO EUTTOM

CALL MOTI (JZFG, JZFP,KFG,KFP, JER, ZFCL, IG)

C MIICFOSTRUC IUFE - READS 4 LINES

3.481 WFITE(KTTYO, 1088$)$

$10 \& 8$ FOFMPTS" TO EYPASS MULT X SCAN TYFE 1 ", Z)

FEE.PCKTTYI, 1086) IH

CALL OVOPN(I7, "MOIFEI.OL", IEFFS)

CALL OVLOD(I 7, GAUSS, IST, IEFFS)

CALL TSCAN (FEF4, JEF)

CALL UVLOD( 17 , I SAPC, I ST, IEFES)

CALL FOUF(IH, PEFI, XEM S, XFF, IEF)

CPLL OVL.OD(I 7 , JPCOEJ, IST, IEPRS)

$J E R=$ !

CALL. MOT2(IG, JEF, KF G,KFF, JE, JF, NSG, NSF)

9C CALL OVLOII T, TAYLOF, IST, IEFPS)

NFTS $=64$

PEF.3 $=92504$.

CALL YSCAN (NPTS,PERZ, IER)

CALL OVLOLCI7, KINGS, IST, IERPS)

IF(IEFFS-1) $13,655,13$

C55 CALL XYSCAN(FEFE,JEF)

CALL ICOUT (KE3, O, IEF)

SO TO 15

18 WEITECKTTYO,1U21)

1021 FORMAT ( $1 \mathrm{H}, 1 \mathrm{X}$, "TYPE O TO END OUTPUT",',2X,

1"TYPE 1 TO PFINT DATA", ', ZX,

2"TYPE 2 TO MEASUFE ANOTHEF SAMPLE ON ELOCK",, $2 X$, "TYPE 3 TO 3"STORE CFTA ON DISK", ', 2X, "TYPE 4 TO MES SUFE NEW SAMPLE PLUC 4,1 , $2 X, "$ TYPE 6 TO FENEASUFE VAVEF FONT"?

15 VRITE (KTTYO, 1 II'? )

1022 FOFMAT (" OUTPUT NUMEEF (TYPE 5 TO LIST OUTFUT OPTIONS? " FEAL (KTTYI, 10U5) IE

1005 FORMAT $T(1)$

$I P=I E+I$

L'RITECKTTYO, 1005) IE

60 TO $(0,10,360,14,348,18,99)$ IE

C THE NANUGL STAGE MUST EE RESET TO TEST OFFAXIS LENSES

30 $X D Q=5.2+X I D(20) 110$.

VFITE(KTTYO, 300U) XDQ

3000 FORMAT(" SET MANUAL STACE TO ", FE."2," CM")

PAUSE

C. MCUE STAEE TO GET MICFOSTEUCTUFE

C JEF=1 MOVES STAGE FFOM VICFOSTFUCTUPE FOSITION TO XYSCAN FC:

C JER-1 MOUES STAGE IN OFFOSITE IIFECTIOM

$J E F=-1$

CALL OVLOD I 7 , JACOFI, IST, IEPFS)

CALL MOT'CIG, JEF, KFG,KFF, JG, JF,NSQ,NSP)

GO TO 3481

O WRITE (KTTYU, IU:3)

1 U23 FORMAT (" END OF PFOG RAM")

CALL FE SET

STOP

10 VRITE (KTTYO, 1024)

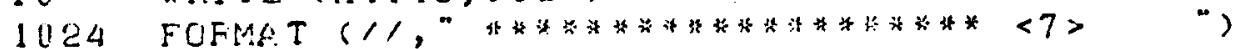

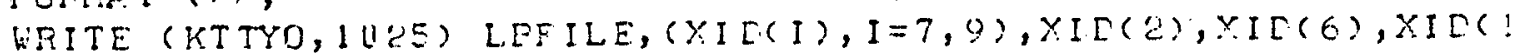

$1(X I D(1), I=10,11), X I[(13), X 1[(16), X I[19)$

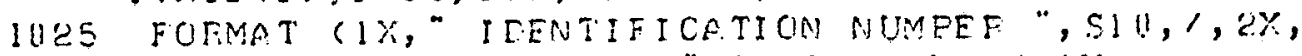




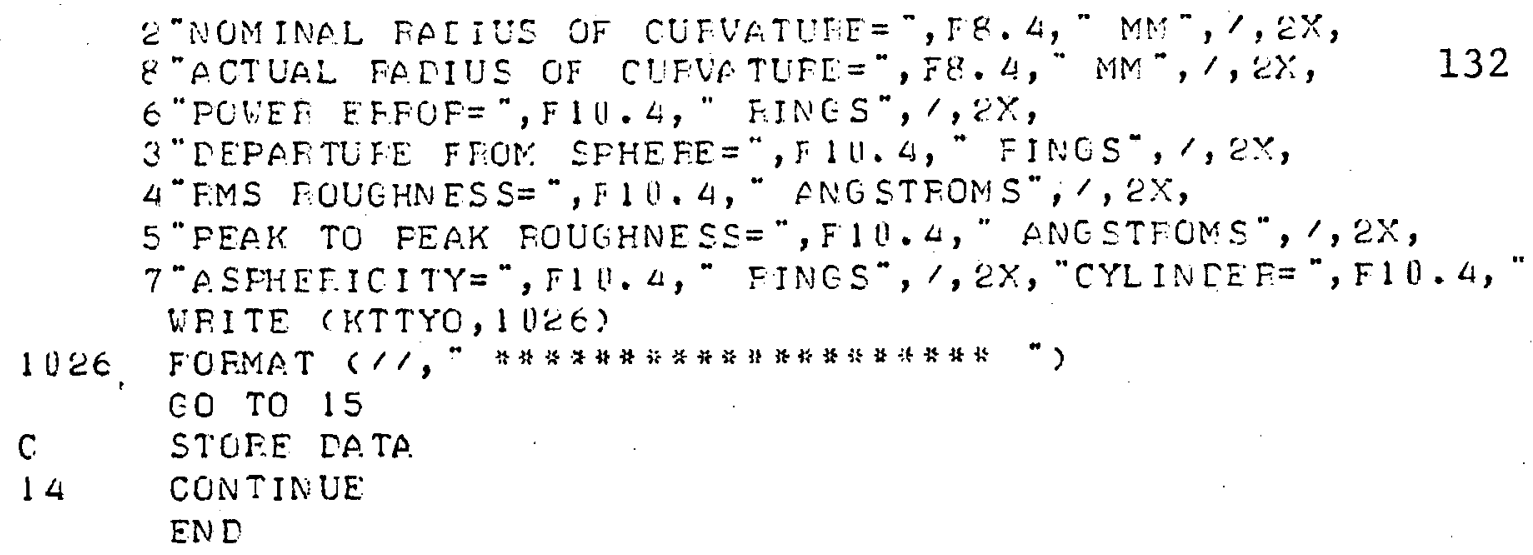


CALL SCALECINGTS, LOX, LNINMKI, MAAX)

IF(JEF) $771,77,77 \%$

771 VRITE(KTTYO, YUEO) JEP

9069 . FOFIMT ${ }^{\circ}$ EFFOF IN PHASE MEASUFEMENTS. JEF=", I3? EO TO 37

772 IF (JER-1) 77,77,771

77 CALL. OVLOLCI I, DOVE, IST, IEFFS)

$K E R=5$

- IF(IERRS- 1) 13,343,13

343 NPTS $=-N P T S$

CALL FIT(NPTS,E, IET, XTIL, YTIL, FOH, CYL, ASFH, XSH, YSH)

GO TO 381

DO $38 \quad L L=1,4$

CALL DEOUT (KE3,MK4, IER)

CALL CDOUT (KE3, MK1, IEP)

38 CONTINUE

381 CONTINUE

IF (EET) $784,783,784$

$783 \quad X P P(L)=0$.

$X F M S(L)=0$.

784 DO $78 \quad I=1,7$

78. $\quad E P(I)=S N C L(E(I))$

C EYPASS OPTION TO FEMOVE TILT ANC FOHEF $I G H=0$

W.FITECKTTYO, 3040 )

3140 FORMATS" TO FEMOVE TILT AND POVER TYFE $1 ", Z$ )

FEPICKTTYI, IU\&E) IGH

CALL OULUD (I 7 , FAERY, I ST, IEFFS)

$K E F=6$

IF(IEFPS-1) 13,344,13

344 CALL LEV(ER,NFTS, PF, FMS, XSH, YSH, IGH)

$X P P(L)=P P$

$X F M S(L)=F M S$

WRITE(KTTYO,1080) PF, RMS

IU80 FOPMATS ${ }^{\circ} \mathrm{P}-\mathrm{P}={ }^{\circ}, F 10.6,{ }^{\circ}$ FNS $\left.={ }^{\circ}, F 10.6\right)$

383 VRITE(KTTYO, 2086)

2086 FOPMATK " TO PLOT OUTPUT TYFE \& ", Z)

FEAD (KTTYI, 1086) IGG

1086 FORMAT (II)

I $F(I G G-1) 36,373,36$

$373 \quad 1 Q(1)=1$

NQPT $S=N P T S+1$

$X(N Q P T S)=X$ (NFTS)

PH (NGPTS $)=F H(N F T S)+1.0$

CALL SVIAF("SUERAPH. SV", IEP)

GO TO $3 \& 3$

36 VPITE(10,3005)

3005 FORMATC " STOFE DATA IN DISK FILE $(1=$ YES; $(1=N O) ? ", Z)$ REAC $(11,300 \&)$ IFIL

3008 FOFNAT (I I)

IF (IFIL) $37,37,40$

40 VIFITE $(10,3000)$

3000 FOFMATS " NAME OF TATA FILE? -;Z)

FEAD 11,3 U1U)DTAFL

3010 FORMAT (SE)

CALL CFILIV DTAFL, 1, IAR)

CALL OPEN (1, DTAFL, 3, IAR)

VIFITE(1,302U)NPTS

3020 FORMATC" ", IS)

DO IO $I=1$, NFTS

10. WRITE(1,3030)X(I), PH(I)

3030 FOFMAT(" ", 2F9.4)

CALL CLOSE ( 1, IAF)

37 CONTINUE

C FADIUS OF CUPVATURE 
$S U M F=X F M S(1)$

SUM: $=X P F(1)$

LO $371 \mathrm{LL}=2, \mathrm{NL}$ $S U M=S U M+X F P(L L)$

371 SUMF $=S U M F+X F M S(L L)$

$X I D(11)=S U M / N L$

$X I D(13)=S L T R / N L$

15 RETURN

END 
V: CVETLEY AFEE SUEFCLTINE STEETCKRZ, JF,IEF?

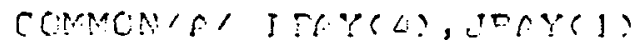

COMYCN, TE, LEFILE(II), IEEILE,LCFILFCIU:

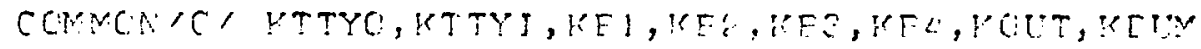

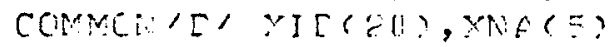

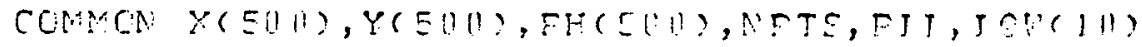

CIMENSICH ULCE), EE(E)

ICLELE FFECJEICH EG:, [ET

KIS ISI

IET $=1$

in $[1=1 !$

$M E r=r k$

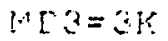

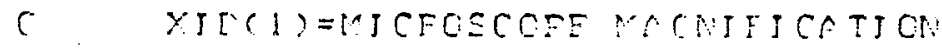

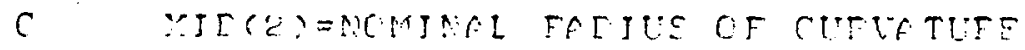

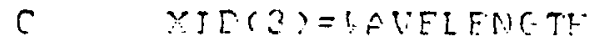

C $\quad X I[C(\angle)=$ GOEYINE IISTENCE

C YIT(S)=CALIEFATEL ZEFC:

C YILCE)=FATIUS CI CUEVATTIFE

C $\quad X T[(7)=Y C N T E$

C $\quad \forall \Gamma(\hat{i})=[A\}$

C XILCU) $=Y A E F$

C $\quad \therefore I[(1 \|)=$ EFFFFTUFE FFOM SEHFFF

C $\quad X I[(11)=5 N S$ ECUCYNES

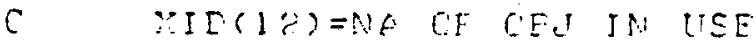

C YIT(13)=FFAK TC FEFR FOLCHAESS

C XIT(14)=FCUEF ETELT

C XITCLS =LENS FAEILS

C $\quad$ YII $(16)=$ STFEFTCITY

C YIIC(1):

C YIECIK)=ACTUFL FCSITION

C XITS IO : $=$ CYLINIEF ETEDF

C XIL COH: $=$ EIOCK HEISHT IN MM

C:CNTINEE

$\therefore I[(T)=11$.

$X \mathrm{~J} D\left(\varepsilon_{0}\right)=0$.

$X \operatorname{II}(\mathrm{C})=0$.

YIT $[5)=41.5$

$\operatorname{xic}(1)=\operatorname{xin}$.

$x \operatorname{II}(12)=11 . \angle 0$

$X \operatorname{II}[(4)=4.7$

$x I[(15)=1$.

$\therefore I\left[\left(c^{\prime}\right)=-1\right.$.

$X I L(E)=X I \Gamma(2)$

$x I[(3)=11.6328$

CALL IECUTCKE 2, NI, IEF?

IF (IPA-IEF) 


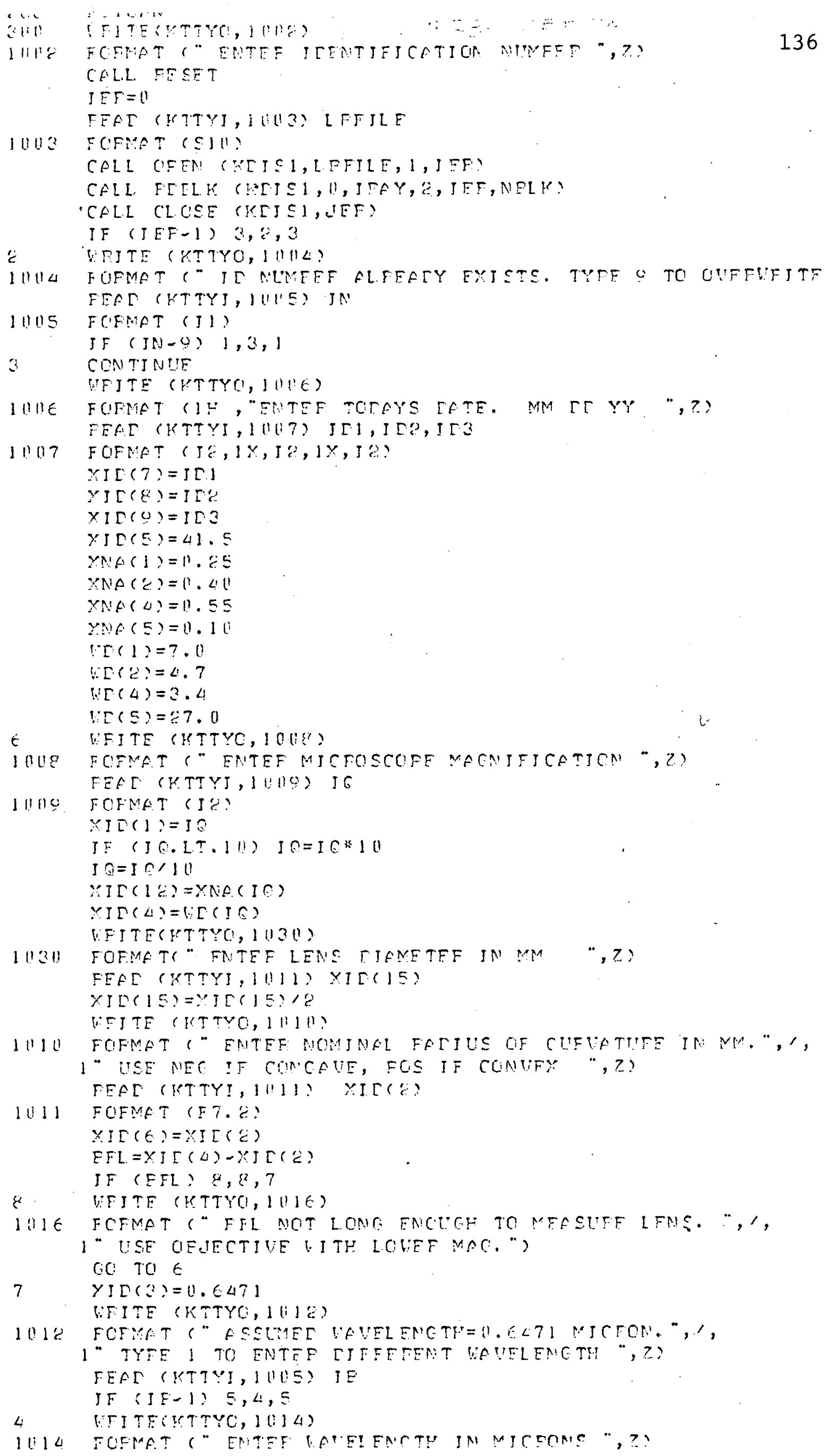




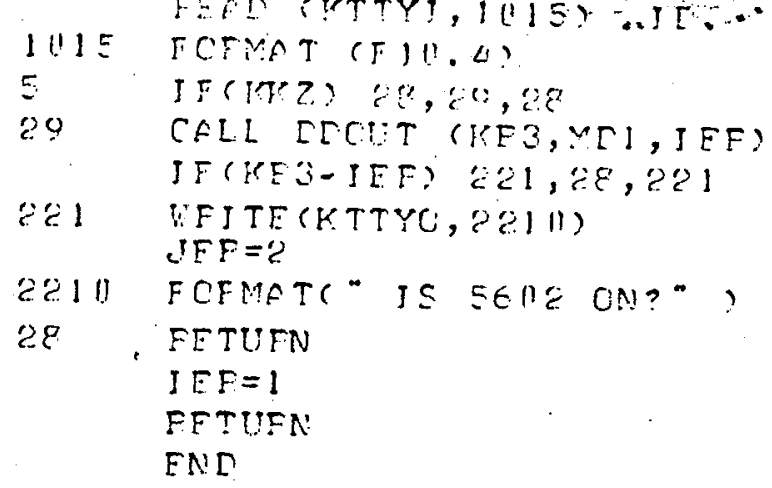

SUEFOLTINE TMETEOSA, N, Y, LFT?

COUELE FFECISJCN $A(7,7), Y(7), A M X$, TENF, DFT CIMENSION ICHEC7)

IET $=1 \cdot[0$

IC $\| K=1, N$

$A M X=[A E S(A(X, B))$

INY $=K$

IO $1 \mathrm{~J}=\mathrm{K}, \mathrm{N}$

IF (TAES(A I,K)),LE. ANY) CO TO 1

FUX $=$ LAES(A $(\mathrm{I}, \mathrm{K})$ ?

$J M X=I$

1

CONTINLE

IF (EAES(AMY), CT. 1. [- LI) EC TO ? $[E T=0 .[\cdot]$

UFITE(1U, IE)

CO TO 14

IF (JMX.FO.K) CO TO 5 $\left[\begin{array}{ll}0 & \triangle\end{array}=1, N\right.$

TEM $F=A\left(Y, d^{\prime}\right)$

$A\left(K, U^{T}\right)=A\left(J H K, C^{\top}\right)$

4

A(IMX, d ) =TEMF

$I C H G(K)=I M X$

TEM $=Y(K)$

$Y(K)=Y(I M X)$ 
$L E 1--1: 0^{\prime}$

C: 0 T TO 6

E $\quad I C H C(K)=K$

G $\quad$ LET $=[E T * A(K, H)$

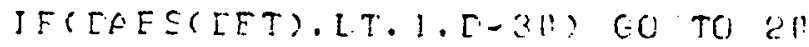

?1 $\quad A(K, K)=1 .[U / F(K, K)$

LO $7 \mathrm{~J}=1, N$

$I F(U \cdot U E \cdot K) \quad A(K, J)=f\left(K, C^{\top}\right) * A(K, K)$

7

CCNT JNUE

$Y(K)=Y(K) * A(K, K)$

IC $\bigcirc \quad I=1, N$

$[0 \& \quad i=1, M$

$I F(J . F G \cdot K)$ CO TO

IF(K.NE.U) $f(I, J)=f(I, U)-A(I, K) * f(K, d)$

8 CONTINLT

$Y(J)=Y(I)-F(I, K) * Y(K)$

- CONTINUE

$[\mathrm{O}$ I $\mathrm{I} \quad \mathrm{I}=1, \mathrm{~N}$

10 CONTINLE

11 CINTINUE

[C $13 \mathrm{~K}=1, \mathrm{~N}$

$\mathrm{L}=\mathrm{N}+\mathrm{I}-\mathrm{i}$

$K I=I C H C(L)$

IF(L.EO.KI) CO TO 13

[C $1 \% I=1, N$

TEM $F=A(I, L)$

$A(I, L)=A(I, K J)$

$12 \quad A(I, K I)=T E M P$

13 CONTINUE

14 FFTUFN

15 FUPMETSEX, "THE MATFIY [ETEFMINSNT IS ZFFO",

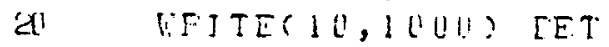

IUUU FUFMATC WFLEE OF EET $=",[14.7)$

0070 21

EMI

CCWEILEF NOSTFC:

CUEFLFY LOUE

SUEFUUTINE FITCNETS, E, IET, YTIL, YTIL, FOV, CYL, ESEL, YSY, YSH?

CCMYON/C, KTTYC,KTTYI, KEI, KE'E, KE: KEL, KOUT, K[TM

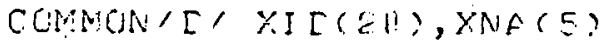

COMMCIN Y(EUU), Y(EUI), PH(EUU), NGFTS, FII, IE(JU)

DOLELE PFECISICN $A(7,7), P(7), Y 1, Y 1, F 1, Y X, Y Y, X Y,[E T$

[CLLELE FFECISICN $Y 3, X M Y, X M N, X S H, Y S H$, POUI, FMX, FYN

IIMENSION EE(7)

I.T: $:(U=0$

$K F=1$

C IF NETS =-, UNE IIMENSICAFL TFFCF-YICFCETEICTHEF TATA

IF (NETS.LT.U) KF=-1

NFTS=IAES(NFTS)

$x ! Y=-1 \cdot \|[-4 \|$

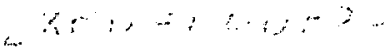




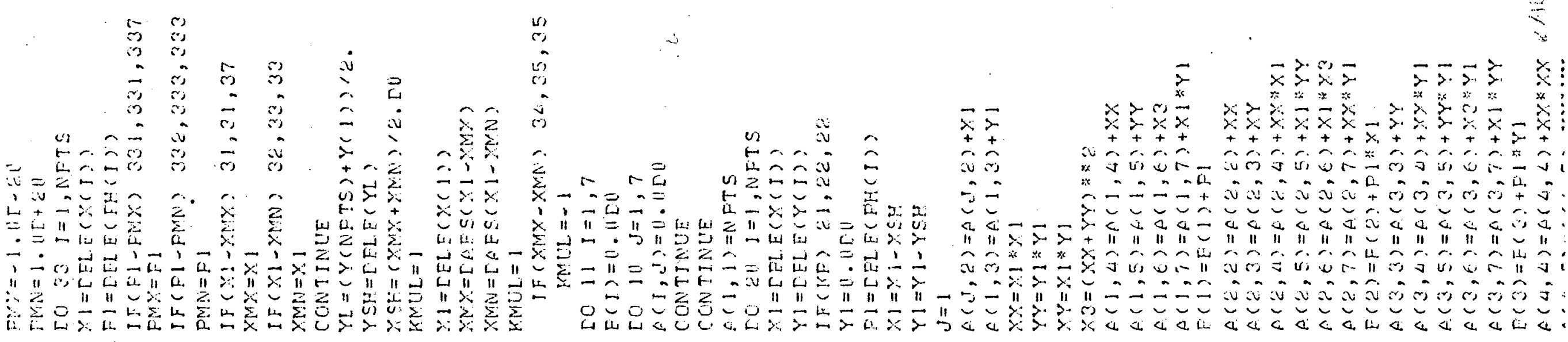

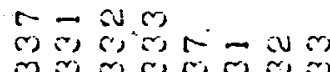

ग)

$\Xi=$

$\overrightarrow{i v}$ 


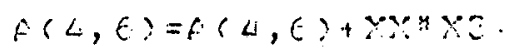

$f(L, 7)=f(4,7)+Y Y * Y \times$

$E\left(\Delta_{i}\right)=E(4)+P 1 * X X$

$E(E)=E(5)+F 1 * Y Y$

$A(E, E)=\theta(E, E)+Y 3 * \times 3$

$A(6,7)=A(\epsilon, 7)+Y Y^{*} \times 3$

$E(\epsilon)=P(\epsilon)+F 1 * X 3$

$P(5,5)=A(5,5)+Y Y * Y Y$

$A(5, E)=A(5, \epsilon)+Y Y^{*} \times 3$

$A(5,7)=A(5,7)+X Y * Y Y$

$f(7,7)=P(7,7)+X Y \because X Y$

¿̇u

$E(7)=E(7)+F I * X Y$

CONTINUE

\section{$N F=7$}

IF (KT I:O-1) $201,110,110$

¿U1 IF(KP) $40, E \|, \in U$

$41 \quad N F=3$

$A(1,3)=A(1,4)$

$f(\varepsilon, 3)=f\left(c^{\prime}, 4\right)$

$A(3,3)=A(4,4)$

$E(3)=F(4)$

EU IO $\quad \& \quad L L=2,7$

$L G=L L-1$

IO $\in C$ L.L.L. $=1, L, 6$

Ë $\quad A(L L, L L L)=\hat{r}$ (LLL,LL)

E1 CONTINUE

IF $(K O L T-\ddot{c}) \& 1 !, \forall 1,81$

(2) $50 \quad 30 \quad I=1, N F$

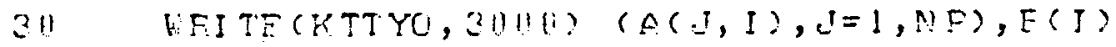

30111 FOFMAT $(7(1 \times, E \& .32,1 \times, E, .4)$

EU CALL TMATESCA,NF,E, LET?

IF (KTBO-1) $\angle 81,481,4 \& 1$

$480 \quad E(T)=R(E)$

$E(6)=\| \cdot[U$

481 JF(KP) $\triangle 8,511,501$

पद $\quad E(4)=E(3)$

$E(3)=0 .[0$

$E(5)=0 .\{[\cdot[\cdot]$

$E(\theta)=0 \cdot[0$

$E(7)=U \cdot[U$

501 IF (KOUT-1) $50,50 \%, 502$

SUC VFITE(KTTYO, 3U11) (E(I), I =1,7)

3001 FCFMAT(" E",7(EO.4,1X))

51 IIO $51 \quad \mathrm{I}=1,7$

E1 $\quad E E(I)=S N(L(E(T))$

THET = STANC ER $(T) /(E E(5)-E E(4):) / 2$

$C=\operatorname{COS}(T H E T)$

$S=S I N(T H E T)$

$\triangle X=E F(4) * C * C+E E(5) * S * S-E E(7) * S * C$

$Y L=S N C L(Y S H)$

$X 1=X M Y-X S H$

$X L=S M C L(X I)$

$Y 1=C F L E(Y(N P T S))$

$E X=F F(4) * S * S+F P(5): C^{k} C+E E(7): S^{*} C$

FHOI $=(X M X-Y S H) * *: 2+(Y 1-Y S F) * *:$

FHO $=$ SNCL (FHOI)

FOH $=(A X+F X): F H O / ?$

$C Y L=(A X-E X) * F H O$

$A S P H=F E(E) * P F O=C F C$

$Y T I L=E E(Z) \times X L$

$Y T I L=E E(3) *(Y(N P T S)-Y L)$

IF (KOUT-1) $115,53,53$

53 UFITECKTTYO, 3UU?) AX, EY, FHO, XTIL, YTIL, FUL, CYL, S SFH

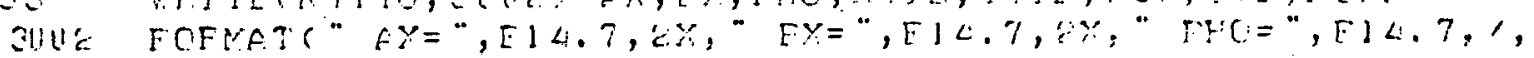
$1-X T S L=", E L 4.7, \because Y, "$ YTIL $=", E J 4.7,1, " P O H=", F \mid 4,7, \because Y$ 


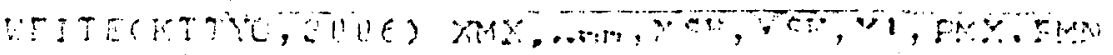

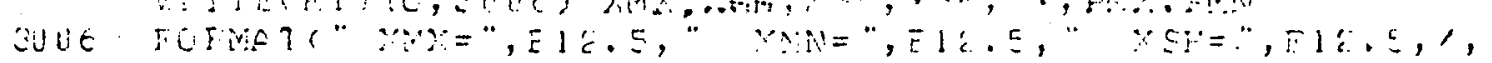

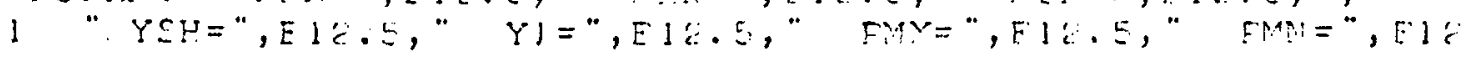
$115 \quad K T \% O=K T C O+1$

IF (KF) $15,15,155$

155 JT (YTUC-1) $35,35,15$

$110 \quad I F(N P-2) \quad 15,15,111$

$111 \quad N F=N F-1$

[C $11: L Z=1, \epsilon$

$11 \varepsilon \quad f(L Z, E)=A(L Z, 7)$

$E(6)=E(7)$

$f(E, E)=6(7,7)$

VIFITE(KTTYC, 3UUS)

3UUS FUFNATC" NO ASFPETE FIT")

CO TO 301

15 FETLFN

ENI 


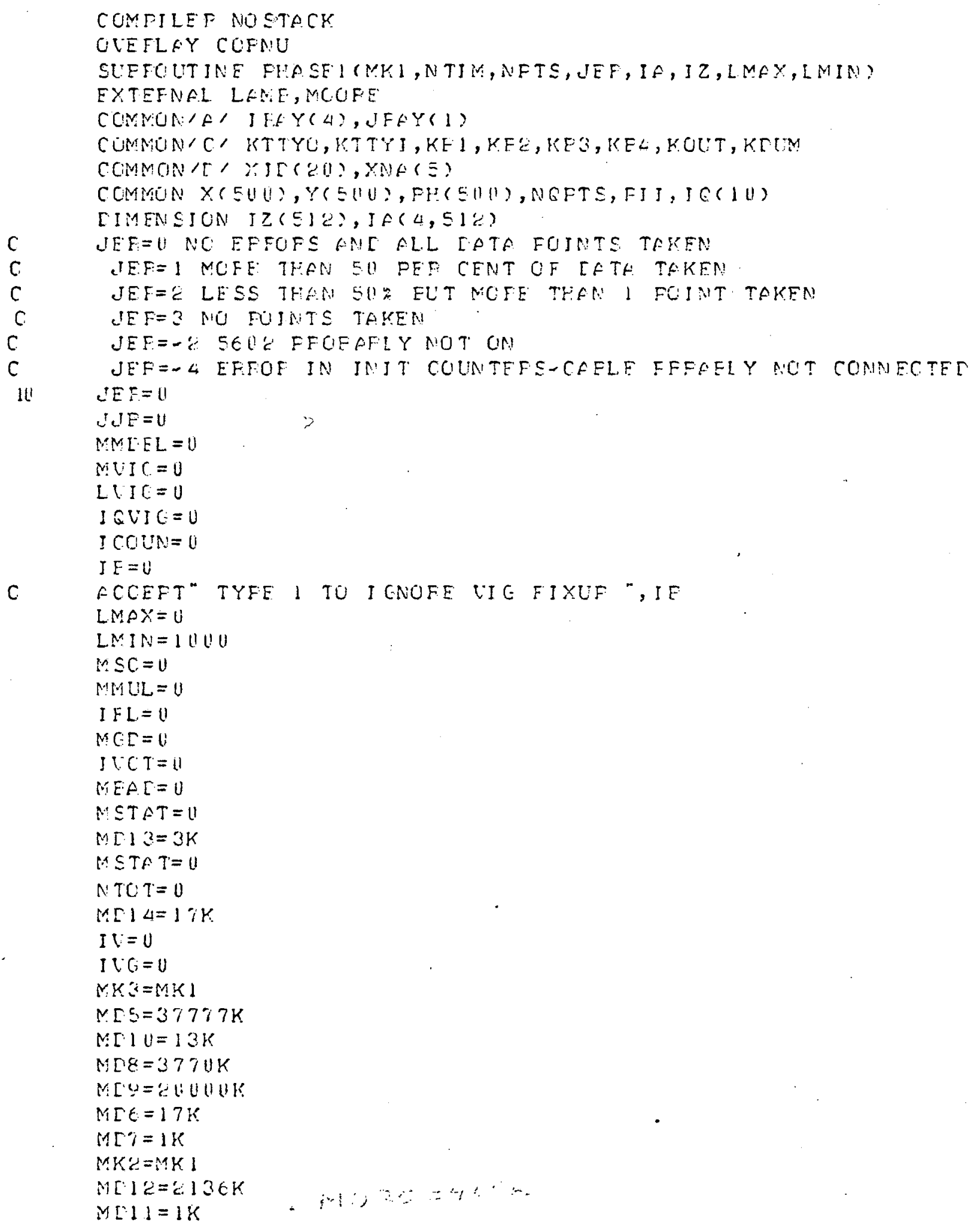




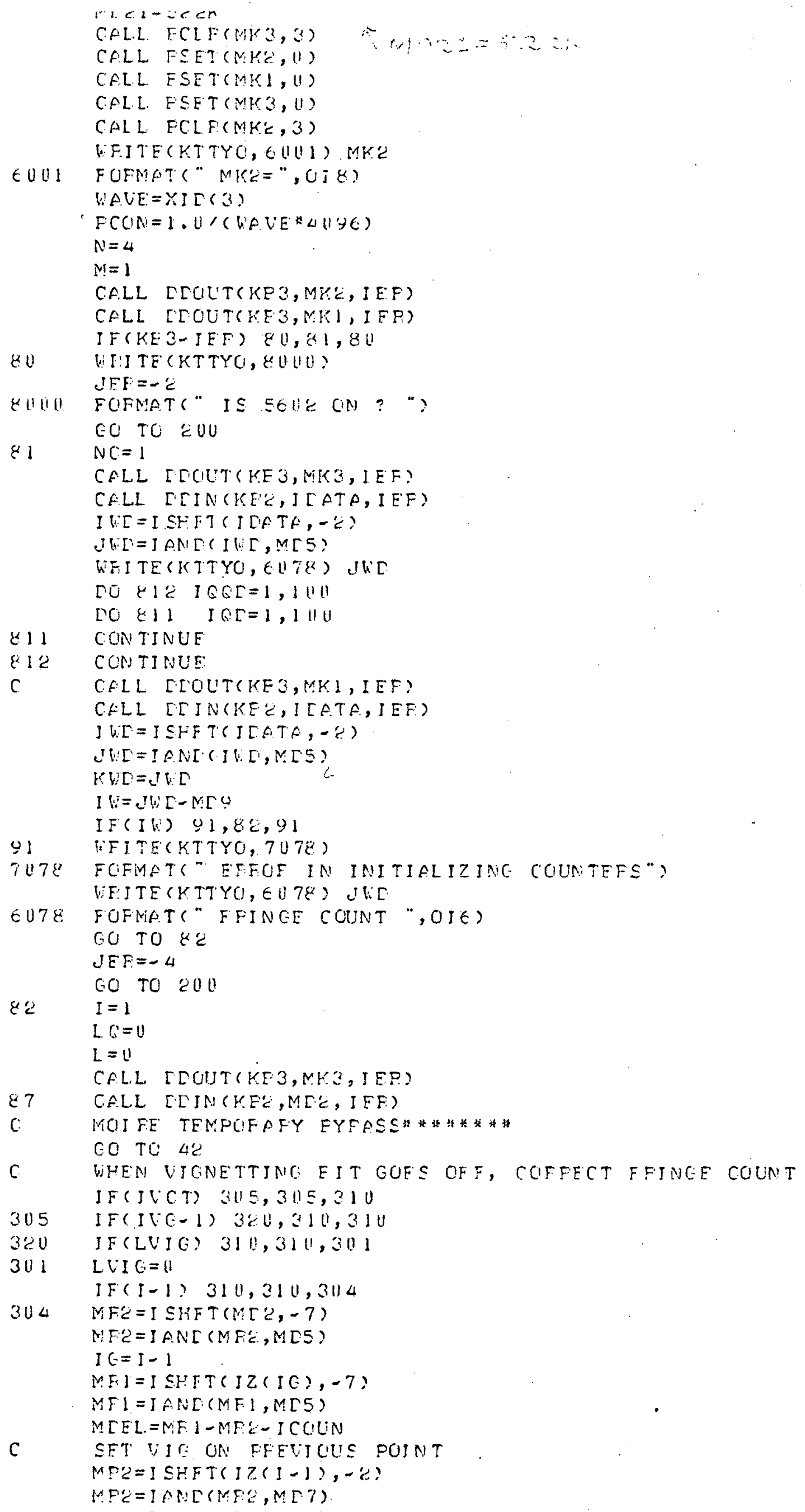


$12(3-1)=12(1-1)+4$

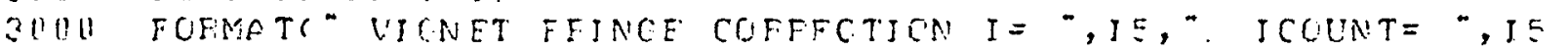

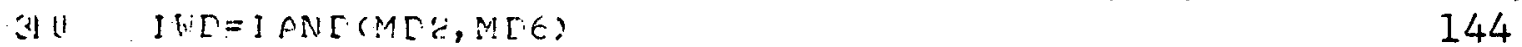

I $H E=I V D-M[1 \|$

IF (IGI) $4 !, 4 \%, 4 !$

$41 \quad L W=I A N\left[\left(M\left[C^{\prime}, M E 7\right)\right.\right.$

LH $\Gamma=L Y \Gamma-M[T$

IF(L.WI) $\in 0, \in 1,61$ )

EU HFITE (KTTYO,7711)

7711 'FCFMATC" EFFOF IN MULTIFLEX ')

$M M U L=M M L L+1$

E 1 MLLISHFT $\left(M\left[C^{\prime},-1\right)\right.$

LUL $=I A N \Gamma(Y[4, M[7)$

L. $: \Gamma=L, V: M-M 7$

CLLE TEMP EYPPSS FOF LLE

GO TO E 3

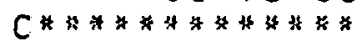

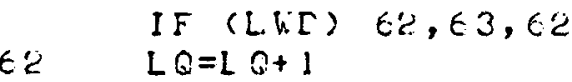

$M G \Gamma=M G \Gamma+1$

$65 \quad L=0$

VEITE(KTTYO,7712)

7712 FOFMAT' EFFCF IN [PTA PJT")

GU TO 71

E3 MI $3=] S F F T(M[2,-2)$

L. LI $=$ IANL $(M[4, M[7)$

L. $\because \Gamma=L U L-M \Gamma 7$

C TENFUFAY EYFASS TO CHECK OUT LLE SYSTFM GCI TC: $\in: 6$

$C * * * * * * \because * * * * * * * 4 * * * *$

$67 \quad L=L+1$

L. VI $G=1$

I VCT $=10$

C SETS A COUNTEF TO AVOIE LATA FOF IVCT COUNTS MVIC $=M V I G+1$

$I F(I V-1) \in U 1, \in \in, \epsilon \in$

691 IF (L. -321100$) \& 7,69,60$

$\in \quad L=0$

I GUIE $=$ I GVI $C+1$

IF(JQVI -1$) \& 7,87,603$

603 VIFITE(KTTYO, 7713 )

7713 FOFMATS" INSTFFICIENT L. IGHT TO MAYF MEFSUFEMENT OF FL IM M: 1 CONNECTET $)$

CC TO $7 \cdot 1$

EE MLU =ISFFT(M[Z, - 3)

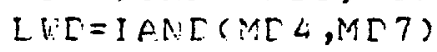

C EYFESS SCEN EIT CHECK IF NOISE UP X SCAN CNLY IF (MK'-M[E!) $6 \in 3,4 \mathrm{C}, 663$

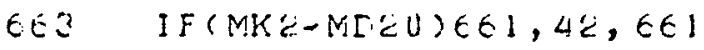

CLLE TEMP EYPASS

C. CO TO $\angle 4$

CE 1 IF $(L[L-M[11) 711,42,70$

$70 \quad M S C=M S C+1$

(ELL. IIOUT (KE3, MKR, JEF)

IF (I-1) 73,73,48

73 IF(MSC-1UUU) 773,773,72

C TELAY

773 LT T $=$ NTIMIS

C. SFEEL UF SCPN TO EET TU EFGINNING CF FFAME $M K G=M K C$ CALL ESET(NHO,E) 
CALL ICOUTCYF3, MKE, IEF?

[C $774 \mathrm{C}^{\prime}=1, \mathrm{~L}, \mathrm{~T}$

[0 77S II $=1,110$

775 CUNTINLE

774 CONTJNUE

CALL [LUUTCKEA, MKË, IEF)

NVIE $=\|$

$L=0$

E. TO TO $\& 7$

$7 \%$ WFITE(KTTYO,7721)

7721 FOFMAT(" EFTOF IN SCAN FIT")

771 IF (KOUT-1) $201,71,7 \mathrm{i}$

71 UFITECKT TYO, 77CU, MML, MC I, MUIE, MSC, NEA D, MSTAT,NTOT

T7É FOFMATC" NO OF EFFOFS IN TPTA ACGLISTION",

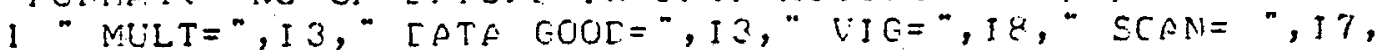

¿" DIFFEFENT $="$, J $3,1,-$ STATUS ", I\&, ¿̇X,

3 "TOTAL NO OF FOINIS FEAL", IE? $J F F=U$

7724 JEF $=3$

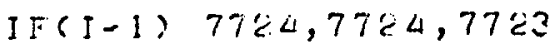

CO TO 200

7723 IL.ON=NTS'

IF (J-ILQ) 77\%

7726 JEF $=2$

60 TO 2011

7725 ILG $=(N F T S * 9) / 10$

$I F(I-I L G) 77 \mathrm{C} 7,201,200$

$77 \check{\complement} 7$ IEF $=1$

GO TO 200

$42 \quad I E F=K E 1$

C CFEUUK FOF STATUS EIT

$M F C=I S H T T(N[C,-4)$

MFE = IAND(MFE, MII3)

CLLE TEME EYFASS FOF LLE GO 104 ' 'C'C

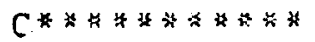

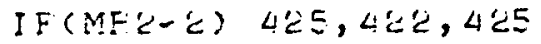

$4 \mathrm{ES}$ MSTAT=MSTFT+1

I VC: $T=1 \mathrm{U}$

$L V I G=1$

IF(MSTHT-EUUUU) $27,87,426$

L¿E VFITE(KTTYC, $4 U U R)$

4008 FOFMAT “ EFEOF IN STATLS EITS")

GE TO 771

$422 \quad I V C T=I V C T-1$

IF (IVCT) $4 \xi 1,4 \dot{z}, 429$

$4 c y \quad$ LVIG $=1$

GO TO 87

421 CONTINLE

CALL. XEAY (IFAY,N, IEF)

CALL IDIN (XEC,MI3,IEF)

LWI $=$ IAN $[$ S $M[3, M \Gamma E)$

NTOT $=N T O T+1$

J. $D=L$ L: $L-M[10$

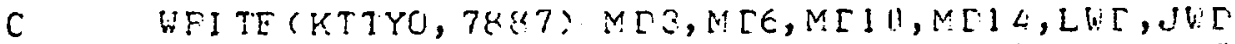

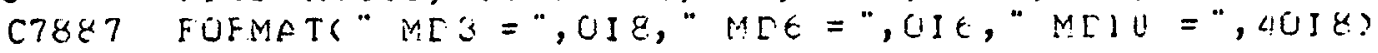

CLLE TEMF EYFA SS GO TOI 44

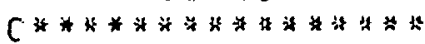

I F (Jh. [.) 43, 44,43

43 IF (IVi-1) 43?, 433,433

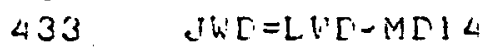

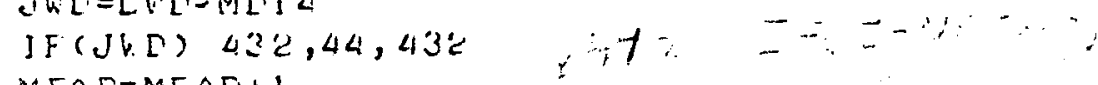

$43 \div$ MEFE=MEF[+1

If (MEFI-1 1000$) \& 7,87,442$ 


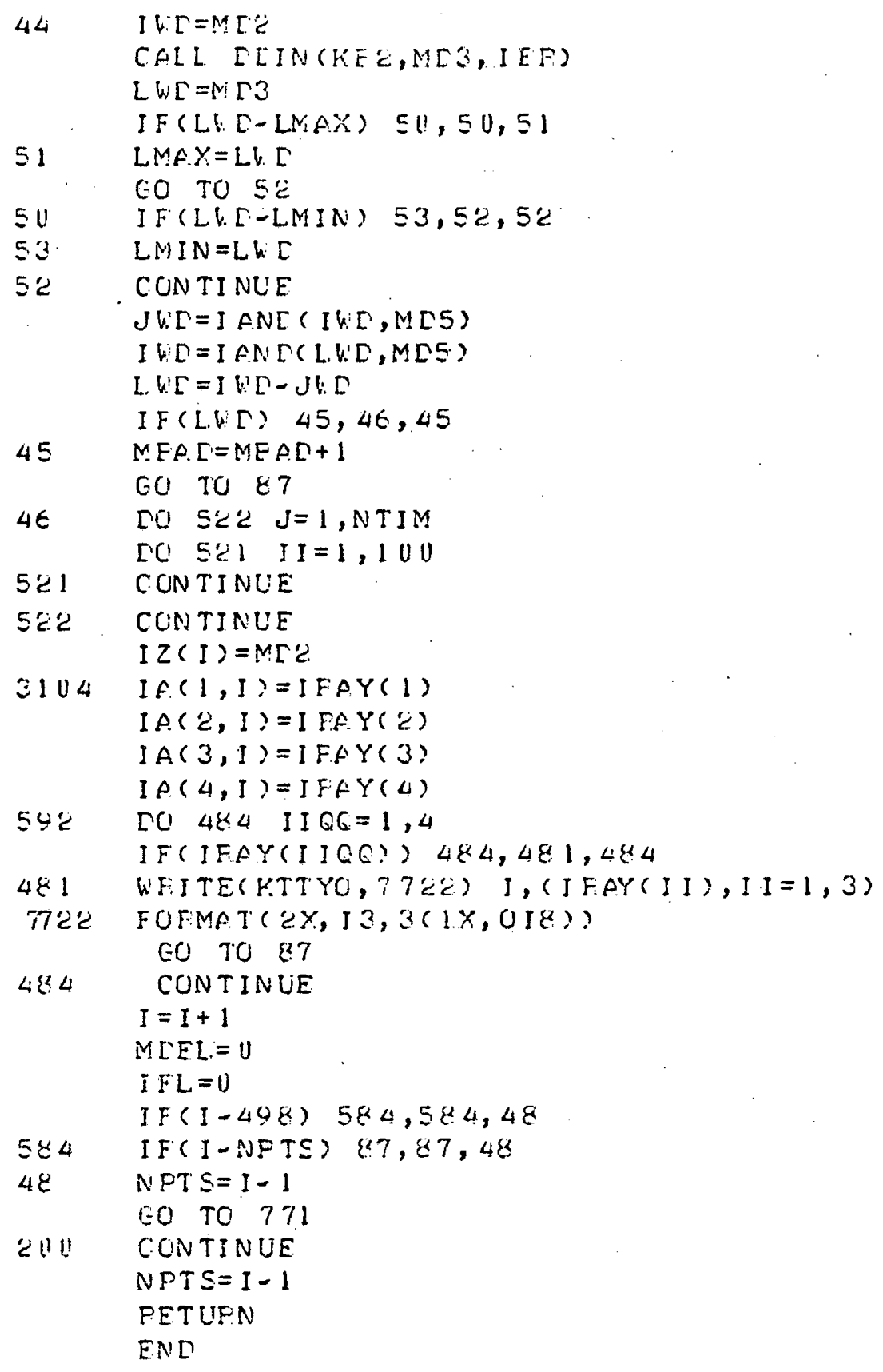




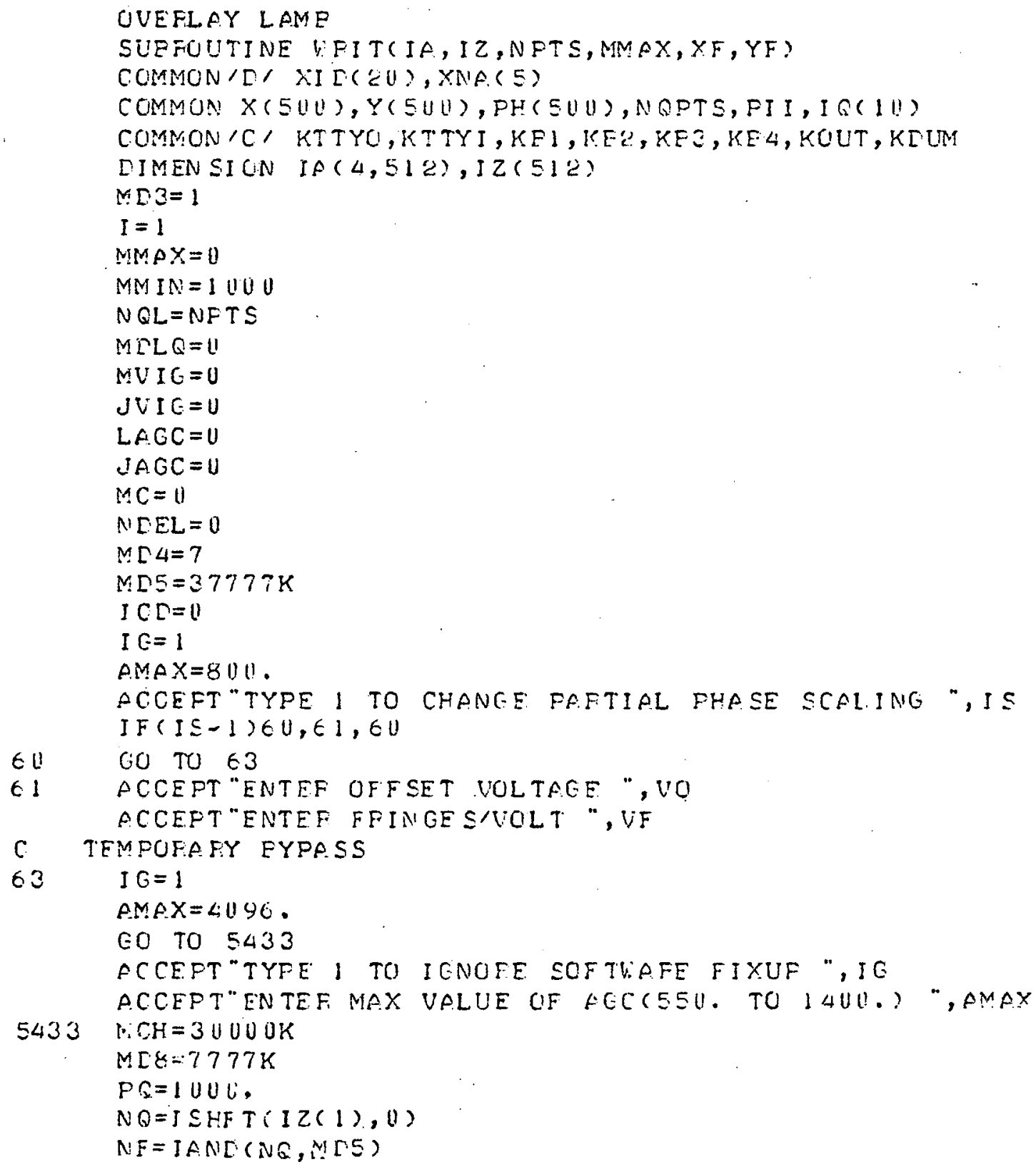




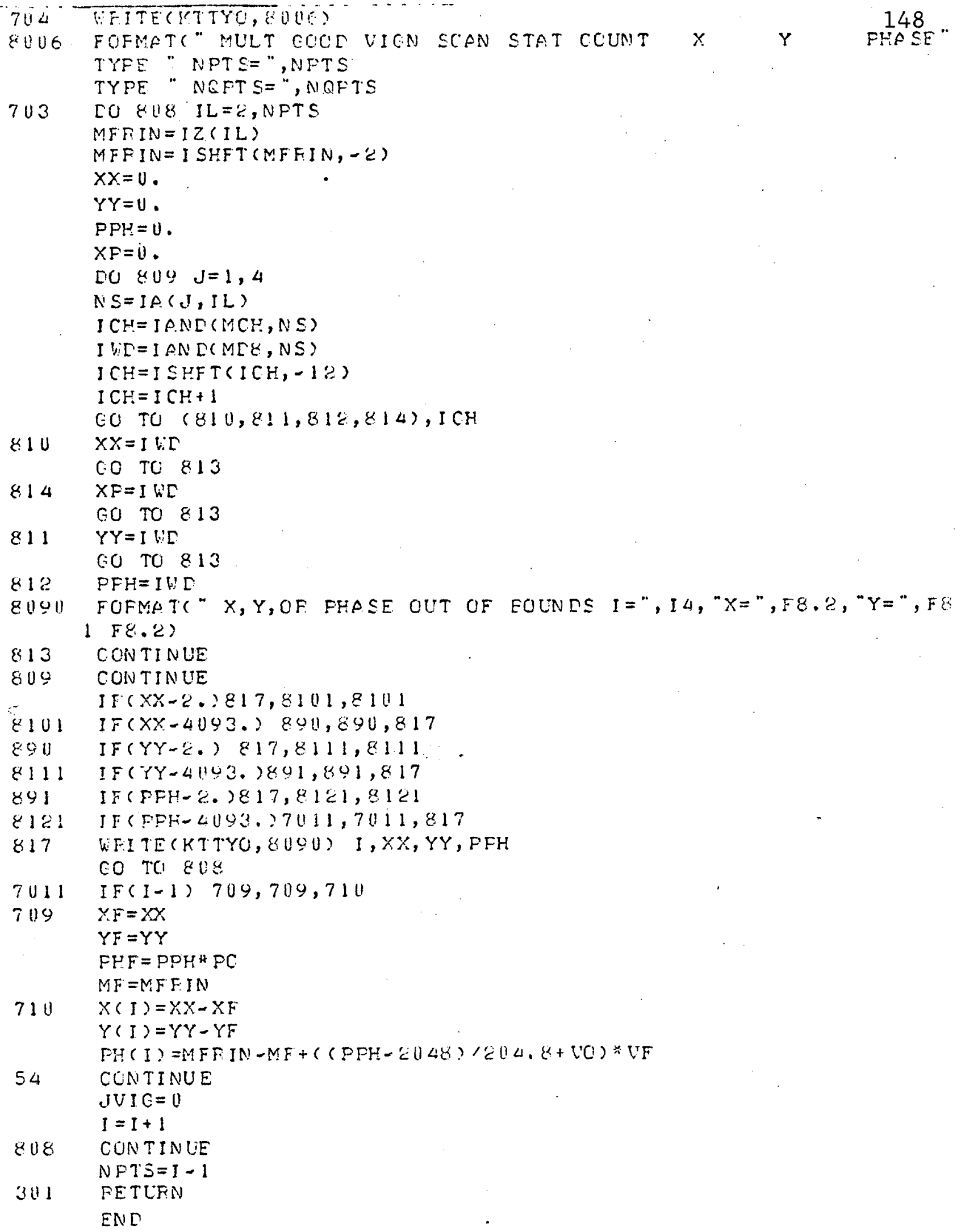




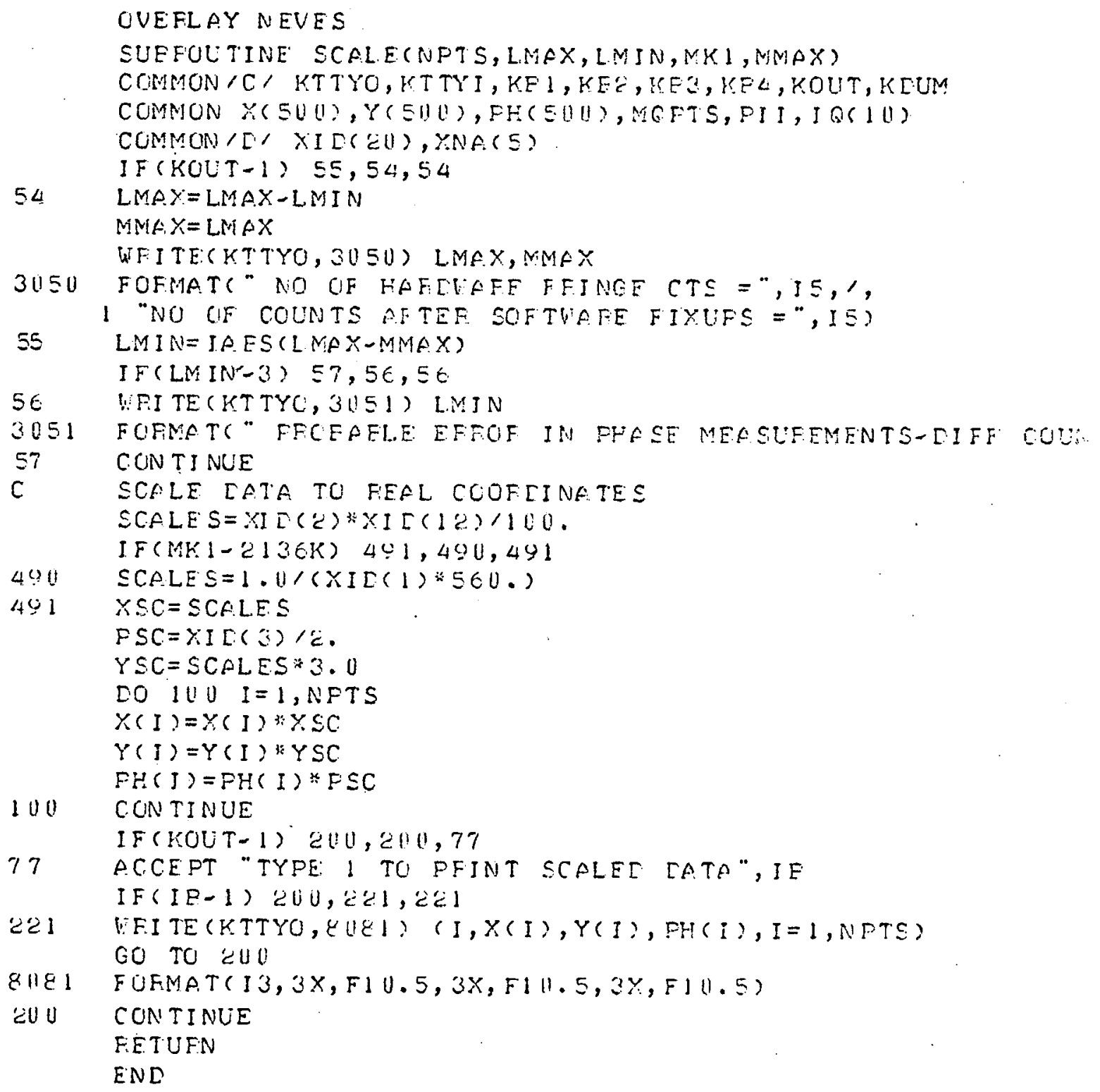


APPENDIX 3

\section{OPERATING INSTRUCTIONS}

I. Optical Alignment

1) Adjust the Fabry-Perot interferometer so there is less than a half fringe across the center $5 \mathrm{~mm}$ of the aperture.

2) With the grating removed and the object in place trigger the oscilliscope from the oscillator output on the front panel. Examine the output of U-18 on board A-1. Focus lens LI in the scanning system until there is no $2 \mathrm{kHz}$ signal present on the scope. Lens LI should now be about $11 \mathrm{~cm}$ from the mirror on the resonant scanner.

3) Install the grating and move the motor driven stage by hand until good contrast fringes are observed. Focus the fringe pattern on the detector.

II. Electronic Adjustments

1) With the scope triggered from the oscillator output place the detector to one side of a dark fringe and observe the output of U-18 on Al. A good clean $2 \mathrm{kHz}$ signal should be observed. Now move the detector to the other side of the fringe. The 
$2 \mathrm{kHz}$ signal should be of the same magnitude but shifted $180^{\circ}$ in phase. If this is not the case refocus lens L1.

2) On board A2 compare the output of U-11 pin 4 with the oscillator signal on the front panel. These two signals should be in phase if they are not adjust the PLL trimpot on A2.

3) Still on board A2 examine the output of U3 pin 6 . This is the rectified error voltage. As the detector is scanned across a fringe this signal should go from a maximum, down to zero and back up to another maximum. The two maximums should be of equal amplitude, if they are not, adjust the OFFSET trimpot on A3. The amplitude of the error voltage should be about 10 volts, this is adjusted by the GAIN trimpot on $\mathrm{A} 3$.

4) Turn on the motor by switching the switch on the speed control box to the low position. The stage will drive to locate either a minimum or a maximum depending on whether the motor direction switch is in the reverse or forward position respectively.

5) Translate the detector in the image of the fringe pattern, the image of the fringe should follow the detector. If the fringe does not follow the detector equally well in both directions then carefully adjust the OFFSET trimpot with the motor off and try again. 
6) Place the scope probe on UI pin 4 of the Fringe Counter board. Make sure the motor is turned off and rotate the motor shaft by hand. Adjust the trimpot on the Fringe Counter Preamplifier until count pulses are observed. Stop turning the motor shaft and lightly tap the table, no pulses should be observed, if they are then adjust the trimpot slightly and try again.

7) Move the scope probe to U-12 pin 6 on the Fringe Counter board, this is the analog position output. Turn on the galvanometer at a very slow scan rate (15 seconds per scan). Set the motor direction on the speed control box to Reverse and when the image of a dark fringe passes over the detector turn on the motor. Drive the horizontal deflection of the oscillascope with the X-GAL output on the front panel. The scope will now display the profile of the object. If the motor breaks into oscillation about a fringe turn the GAIN trimpot on board A3 counterclockwise slowly until it stops (usually less than $1 / 8$ turn).

At this time the data acquisition program MOIRE I may be run. 
REFERENCES

1) Rayleigh, "On the Manufacture and Theory of Diffraction Gratings," Philosophy Magazine, 54, Vo1. 47, No. 310, 81-93 (Feb. 1874)

2) Anderson, J.A., "The Manufacture and Testing of Diffraction Gratings," Glazebrooks Dictionary of Applied Physics, 4, 37 (1923).

3) Brewer, G.A., "Photo Grid Methods," Photographic Engravers' Bulletin, 22, 15 (1932).

4) Merton, T.,"On the Reproduction and Ruling of Diffraction Gratings," Proceedings of the Royal Society of London, A 201, 187 (1950).

5) Meadows, D.M., Johnson, W.O., Allen, J.B., "Generation of Surface Contours by Moiré Patterns," Applied Optics, 9, 942 (1970).

6) Takasaki, H., "Moiré Topography," Applied Optics, 9, 1467 (1970).

7) Miles, C.A., Speght, B.S., "Recording the Shape of Animals by a Moiré Method," Journal of Physics, E 8,773 (1975).

8) Wasowski, J., "Moiré Topographic Maps," Optical Communication, 2, 321 (1970).

9) Idesawa, M., Yatagui, T., Soma, T., "Scanning Moiré Method and Automatic Measurement of 3-D Shapes," Applied Optics, Vol. 16, 8, 2152 (1977).

10) Erf, R.K. (ed), Holographic Nondestructive Testing, Academic Press, New York, 1974, pp. 118-119.

11) Johnson, G.W., Moore, D.T., "Design and Construction of a Phase-Locked Interference Microscope," SPIE, Vo1. 103, 76 (1977).

12) Ibid.

13) Post, D., "Analysis of Moiré Fringe Multiplication Phenomena," Applied Optics, 6, 1938 (1967). 
14) Post, D., Gurland, J., "Sharpening and Multiplication of Moiré Fringes," Experimental Mechanics, 1, 154 (1967).

15) Theocaris, P.S., "Isopachic Patterns by the Moire' Method," Experimental Mechanics, 4, 153-159 (1964).

16) Haines, K.A., Hildebrand,H.P., "Multiple-Wavelength and Multiple-Source Holography Applied to Contour Generation," JOSA, 57, 155 (1967).

17) Gradient Index Laboratory, "Phase-Locked Interference Microscope Manual," University of Rochester, The Institute of Optics (1977). 


\section{BIBLIOGRAPHY}

Anderson, J.A., "The Manufacture and Testing of Diffraction Gratings," Glazebrooks Dictionary of Applied Physics, 4,37 (1923).

Brewer, G.A., "Photo Grid Methods," Photographic Engravers' Bulletin, 22, 15 (1932).

Chiang, C., "Moire' Topography," Applied Optics, 14, 1, 177 (1975).

Erf, R.K. (ed), Holographic Nondestructive Testing, Academic Press, New York, 1974.

Gradient Index Laboratory, "Phase-Locked Interference Microscope Manual," University of Rochester, The Institute of Optics (1977).

Guild, J., Interference of Crossed Diffraction Gratings, Clarendon Press, Oxford, England (1956).

Haines, K.A., Hildebrand, H.P., "Multiple-Wavelength and Multi-Source Holography Applied to Contour Generation," JOSA, 57, 155 (1967).

Idesawa, M., Yatagui, T., Soma, T., "Scanning Moiré Method and Automatic Measurement of 3-D Shapes," Applied Optics, Vol. 16, 8, 2152 (1977).

Jaerisch, W., Makosch, G., "Optical Contour Mapping of Surfaces," Applied Optics, 12, 1552 (1973).

Johnson, G.W., Moore, D.T., "Design and Construction of a Phase-Locked Interference Microscope," SPIE, Vo1. 103, 76 (1977).

MacGovern, A., "Projected Fringes and Holography," Applied Optics, 11, 2972 (1972).

Meadows, D.M., Johnson, W.O., Allen, J.B., "Generation of Surface Contours by Moire' Patterns," Applied Optics, 9,942 (1970). 
Merton, T., "On the Reproduction and Ruling of Diffraction Gratings," Proceedings of the Royal Society of London, A 201,187 (1950).

Miles, C.A., Speght, B.S., "Recording the Shape of Animals by a Moire' Method," Journal of Physics, E 8, 773 (1975)

Moore, D.T., Eastman, J.M., "Practical Aspects of A.C. Moire' Fringe Interferometry," OSA Annual Meeting, Houston, Texas (October, 1974).

Post, D., "Analysis of Moire' Fringe Multiplication Phenomena," Applied Optics, 6, 1938 (1967).

Post, D., Gurland, J., "Sharpening and Multiplication of Moire'Fringes," Experimental Mechanics, 1, 154 (1967).

Rayleigh, "On the Manufacture and Theory of Diffraction Gratings," Philosophy Magazine, 54, Vo1. 47, No. 310, 81-93 (Feb. 1874).

Sampson, R.C., Campbell, D.M., "The Grid Shift Technique for Moire' Analysis of Strain in Solid Propellants," Experimental Mechanics, 7, 449 (1967).

Takasaki, H., "Moire' Topography," Applied Optics, 9, 1467 (1970).

Takasaki H., "Moire' Topography," Applied Optics, 12, 845 (1973).

Theocaris, P.S., "Isopachic Patterns by the Moire' Method," Experimental Mechanics, 4, 153-159 (1964).

Wasowski, J., "Moiré Topographic Maps," Optical Communication, 2, 321 (1970). 